GEOLOGICAL SURVEY CIRCULAR 232

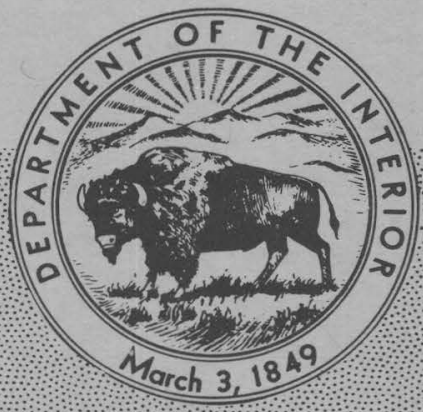

\title{
THE INDUSTRIAL UTILITY OF PUBLIC WATER SUPPLIES IN THE PACIFIC STATES, 1952
}

\author{
By E. W. Lohr, R. T. Kiser C. S. Howard, and I. W. Walling
}



UNITED STATES DEPARTMENT OF THE INTERIOR

Oscar L. Chapman, Secretary

GEOLOGICAL SURVEY

W. E. Wrather, Director

GEOLOGICAL SURVEY CIRCULAR 232

\section{THE INDUSTRIAL UTILITY OF PUBLIC WATER SUPPLIES IN THE PACIFIC STATES, 1952}

By E. W. Lohr, R. T. Kiser C. S. Howard, and I. W. Walling 



\section{CONT ENTS}

\begin{tabular}{|c|c|}
\hline $\begin{array}{l}\text { Page } \\
\ldots \quad 1\end{array}$ & California--Continued .. \\
\hline ....... 3-59 & San Jose...$\ldots \ldots \ldots$ \\
\hline Alameda ....... & eand 1 \\
\hline Alhambra $\ldots \ldots \ldots \ldots \ldots$ & San Mateo \\
\hline Arcadia $\ldots \ldots \ldots \ldots \ldots \ldots \ldots, 4$ & Santa Ana \\
\hline Bakersfield $\ldots \ldots \ldots \ldots \ldots \ldots$. 5 & Santa Barbara ... \\
\hline Berkeley.$\ldots \ldots \ldots \ldots$. & Santa Cruz ..... \\
\hline Beverly Hills $\ldots \ldots \ldots \ldots \ldots$ 6-7 & Santa Monica .. \\
\hline Burbank $\ldots \ldots \ldots \ldots \ldots \ldots \ldots$ 8 & Santa Rosa $\ldots . . \ldots \ldots \ldots$ \\
\hline Compton..$\ldots \ldots \ldots \ldots \ldots$ & South Gate \\
\hline Contra Costa District ..... & Stockton \\
\hline Culver City $\ldots \ldots \ldots \ldots \ldots \ldots, 12$ & .............. \\
\hline East Bakersfield $\ldots \ldots \ldots \ldots \ldots, 13$ & Vallejo \\
\hline East Los Angeles $\ldots \ldots \ldots$ & Whittier \\
\hline Eureka $\ldots \ldots \ldots \ldots \ldots \ldots \ldots, 14$ & Oregon .... \\
\hline Fresno $\ldots \ldots \ldots \ldots \ldots \ldots \ldots \ldots 15$ & $\ldots \ldots \ldots \ldots \ldots$ \\
\hline lale $\ldots \ldots \ldots \ldots \ldots \ldots \ldots, 16$ & Astoria \\
\hline gton Park . ......... & Bend \\
\hline Inglewood $\ldots \ldots \ldots \ldots \ldots \ldots$ & Coos Bay. \\
\hline Long Beach $\quad \ldots \ldots \ldots \ldots \ldots \ldots 19$ & $\ldots \ldots \ldots \ldots$ \\
\hline Los Angeles $\ldots \ldots \ldots \ldots \ldots \quad 20-23$ & Eugene ... \\
\hline olitan Water District & h Fa \\
\hline of Southern California .... & Medford . \\
\hline Lynwood $\ldots \ldots \ldots \ldots \ldots \ldots .24$ & Pendleton $\ldots \ldots \ldots \ldots \ldots$ \\
\hline $\tan$ Beach $. . . \ldots .$. & ind .. \\
\hline ia $\ldots \ldots \ldots \ldots \ldots$ & 70 \\
\hline ey $\ldots \ldots \ldots \ldots \ldots$ & Springfield \\
\hline rey Park $\ldots \ldots \ldots \ldots \ldots 28$ & Washington \\
\hline al City $\ldots \ldots \ldots \ldots \ldots \ldots \quad 29$ & Aberdeen \\
\hline Oakland $\ldots \ldots \ldots \ldots \ldots \ldots 30-31$ & Bellingham \\
\hline Ontario $\ldots \ldots \ldots \ldots \ldots \ldots \ldots, 32$ & Bremerton \\
\hline Oxnard $\quad \ldots \ldots \ldots \ldots \ldots \ldots \ldots, 33$ & Everett $\ldots \ldots \ldots \ldots \ldots \ldots \ldots \ldots$ \\
\hline Palo Alto $\ldots \ldots \ldots \ldots \ldots \ldots \ldots, 34$ & Longview . \\
\hline Pasadena $\ldots \ldots \ldots \ldots \ldots \ldots$....... 35-36 & pia. \\
\hline ........... & Renton \\
\hline Redondo Beach $\ldots \ldots \ldots \ldots \ldots, 38$ & Richland \\
\hline od City $\ldots \ldots \ldots \ldots \ldots 39$ & Seattle \\
\hline Richmond $\ldots \ldots \ldots \ldots \ldots \ldots, 39$ & . $83-84$ \\
\hline Riverside $\quad \ldots \ldots \ldots \ldots \ldots \ldots \ldots 40$ & Tacoma \\
\hline Sacramento $\ldots \ldots \ldots \ldots \ldots \ldots 41$ & Vancouver \\
\hline San Bernardino $\ldots \ldots \ldots \ldots \ldots, 42$ & Walla Walla . \\
\hline San Diego $\ldots \ldots \ldots \ldots \ldots$. $43-44$ & Wenatchee .... \\
\hline San Francisco $\ldots \ldots \ldots \ldots .45-47$ & Yakima .. \\
\hline
\end{tabular}

\section{ILLUSTRATION}

Figure 1. Map of the United States showing sections covered by the nine circulars on the Industrial Utility of Public Water Supplies of the United States, 1952. The shaded portion represents the section of the country covered by this circular 


\title{
THE INDUSTRIAL UTILITY OF PUBLIC WATER SUPPLIES IN THE PACIFIC STATES, 1952
}

\author{
By E. W. Lohr, R. T. Kiser, C. S。Howard, \\ and I. W. Walling
}

\section{INTRODUCTION}

The location of industrial plants is dependent on an ample water supply of suitable quality. Information relating to the chemical characteristics of the water supplies is not only essential to the location of many plants but also is an aid in the manufacture and distribution of many commodities.

Public water supplies are utilized extensively as a source of supply for many industrial plants, used either as delivered for domestic consumption or with further treatment if necessary to meet specific needs of the plant, such as water for processing, cooling, and steam generation. The industrial use of water in the United States in 1950 was estimated to be more than $\mathbf{7 5}$ billion gallons per day from private sources. In addition, about 6 billion gallons per day was estimated to be taiken from public water supplies.

U. S. Geological Survey Water-Supply Paper 658, "The industrial utility of public water supplies in the United States, 1932" contains information pertaining to the public water supplies of 670 of the larger cities throughout the United States. This report, which is still in print and being distributed, has filled an important need in the field of water-supply engineering. The demand for more up-to-date information and more extended coverage has led to studies by the Geological Survey for revision of the information contained in the 1932 report. The revised report, which will include data pertaining to public water supplies of more than 1,200 cities in the United States, will eventually be published as a Geological Survey Water-Supply Paper. However, in order that the information might be available at the earliest possible time, nine preliminary reports are being issued which give data on the larger cities in each state. These nine reports are being released as Geological Survey Circulars, each covering a group of states as delineated by the Bureau of Census in taking the census of the population of the country. (See fig. 1). The reports give descriptive inforamtion and analytical data for approximately three-fourths of the cities that will be included in the final report for each of the states.

This circular is the fifth of the series and includes data for the States of California, Oregon, and Washington. (See fig. 1). The report gives the population (1950) of the city, the population supplied, ownership, sources and treatment of supplies, capacity of treatment plants, storage facilities for both raw and finished waters, and chemical analyses of the water for a total of 80 of the larger cities of California, Oregon, and Washington. The data for each city are essentially the same as will appear in the complete report for the whole country.

Data for the supplies in California, Oregon, and Washington were compiled mainiy by R. T. Kiser, chemist, Salt Lake City, assisted by I. W. Walling, district chemist, Sacramento, for the supplies in California. The work was done under the general supervision of C. S. Howard, regional chemist, Salt Lake City. Review and final assembly of the data were made oy E. W. Lohr in the Washington office under the direction of S. K. Love, Chief, Quality of Water Branch. 


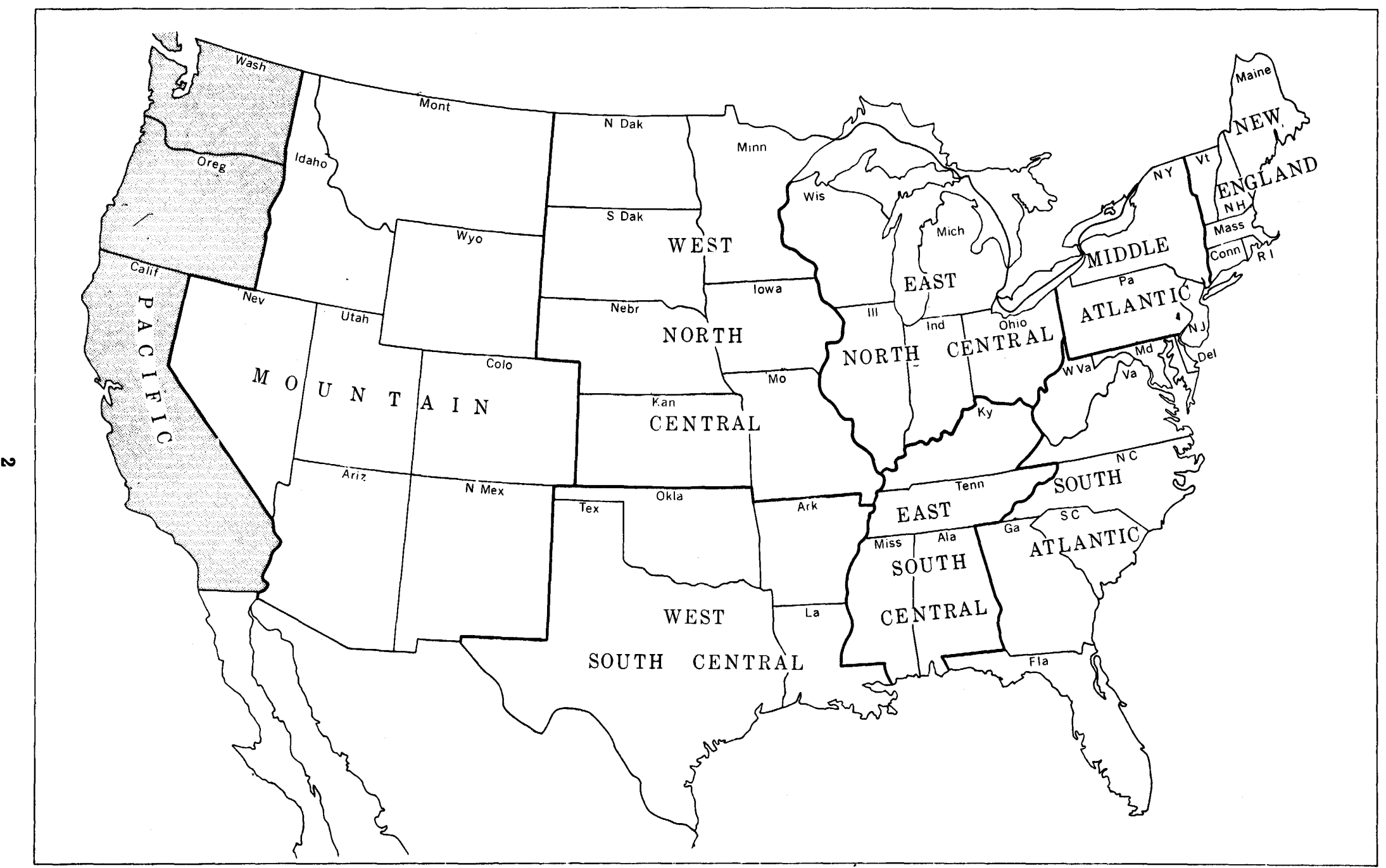

Figure 1. -Map of the United States showing sections covered by the nine circulars on the Industrial Utility of Public Water Supplies of the United States, 1952. The shaded portion represents the section of the country covered by this circular. 


\title{
CALIFORNIA
}

\section{CALIFORNIA}

\author{
ALAMEDA \\ (Population, 64, 430)
}

Ownership: East Bay Municipal Utility District. (See Oakland.)

\section{ALHAMBRA}

(Population, 51, 359)

Ownership: Municipal.

Source: 9 wells ranging in depth from 300 to $872 \mathrm{ft}$. The yield of the wells is reported to be from 900 to $3,150 \mathrm{gpm}$. Emergency supply from wells owned by California Water and Telephone Co. , South Pasadena.

Treatment: None.

Storage: Reservoirs and tanks, 27,675, $000 \mathrm{gal}$.

\section{ANALYSES}

(Analyses, in parts per million, by California State Dept. of Public Health)

\begin{tabular}{|c|c|c|c|c|c|}
\hline & Well 2 & Well 8 & Well 12 & $\begin{array}{c}\text { Longden } \\
\text { Well }\end{array}$ & $\underset{\text { Reservoir }}{\text { Garfield }}$ \\
\hline 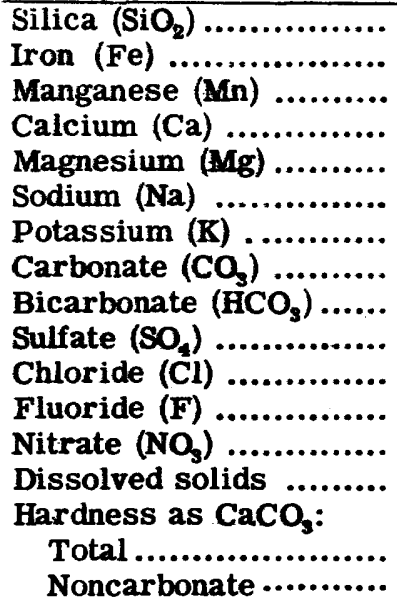 & $\begin{array}{l}{ }^{.-} \\
35^{.00} \\
7.0 \\
19 \\
0^{0-} \\
129 \\
21 \\
13 \\
11 \\
221 \\
115 \\
9\end{array}$ & 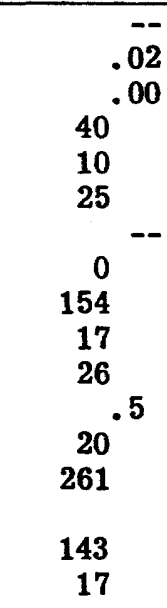 & 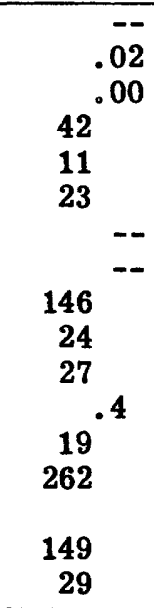 & 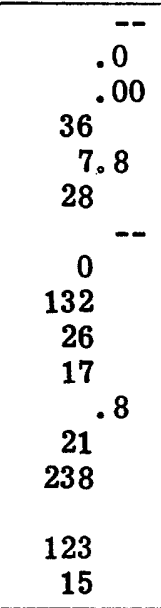 & 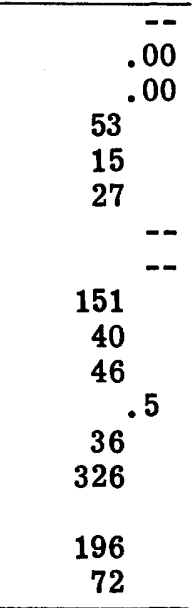 \\
\hline 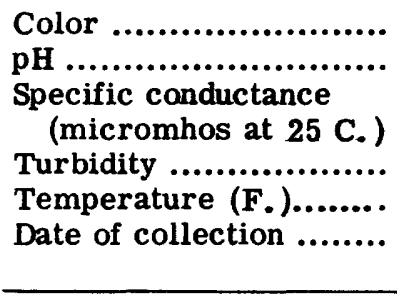 & $\begin{array}{r}7.4 \\
317 \\
-- \\
\text { Feb. } 9, \\
1951 \\
\end{array}$ & $\begin{array}{r}7.3 \\
389 \\
-- \\
-- \\
\text { Feb. } 9, \\
1951 \\
\end{array}$ & $\begin{array}{r}7.3 \\
400 \\
-- \\
-- \\
\text { Feb. } 9, \\
1951\end{array}$ & $\begin{array}{r}7.3 \\
371 \\
-- \\
-- \\
\text { Feb. } 9, \\
1951 \\
\end{array}$ & $\begin{array}{r}7.8 \\
535 \\
-- \\
\text { Feb. } 9{ }^{--} \\
1951^{--}\end{array}$ \\
\hline $\begin{array}{l}\text { Depth (feet) } \\
\text { Diameter (inches)............ } \\
\text { Date drilled ............... } \\
\text { Percent of supply ........ }\end{array}$ & $\begin{array}{r}734 \\
30-18-16 \\
1931 \\
--\end{array}$ & $\begin{array}{r}764 \\
30-20-16 \\
1935 \\
--\end{array}$ & $\begin{array}{r}862 \\
20 \\
1948 \\
--\end{array}$ & $\begin{array}{r}778 \\
26-20 \\
1926\end{array}$ & \\
\hline
\end{tabular}

${ }^{\mathrm{a}}$ Receives water from Wells 8, Longden, and Garfield. 


\section{CALIFORNIA}

ARCADIA

(Population, 23, 066)

Ownership: Municipal.

Source: 11 wells ranging in depth from 462 to $862 \mathrm{ft}$. The yield of the wells ranges from 500 to 3,600 gim. Emergency supply from wells owned by Southern California Water Co.

Treatment: None.

Storage: Reservoirs, 10, 500, 000 gal.

Analyses indicate that there is consideraible variation in the character of the water from the individual wells of approximately same depth and at different depths. The analyses selected show the range in dissolved solids and hardness in the water from the different wells.

\section{ANALYSES}

(Analyses, in parts per million, by Pomeroy \& Assoc., Pasadena, Calif.)

\begin{tabular}{|c|c|c|c|c|c|}
\hline & $\begin{array}{l}\text { Orange } \\
\text { Grove } \\
\text { Well 1A }\end{array}$ & $\begin{array}{c}\text { Orange } \\
\text { Grove } \\
\text { Well 4A }\end{array}$ & $\begin{array}{l}\text { Longden } \\
\text { Well } 1^{\mathrm{a}}\end{array}$ & $\begin{array}{c}\text { Camino } \\
\text { Real } \\
\text { Well } 1\end{array}$ & $\begin{array}{l}\text { Rancho } \\
\text { Well } 8\end{array}$ \\
\hline Silica $\left(\mathrm{SiO}_{2}\right) \ldots \ldots \ldots \ldots \ldots$ & 11 & 12 & 18 & 14 & $\overline{14}$ \\
\hline 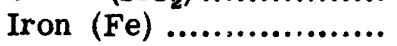 & - & - & -- & -- & -- \\
\hline Manganese (Mn) .......... & -- & -- & -- & -- & -- \\
\hline Calcium (Ca) ............. & 26 & 44 & 48 & 39 & 79 \\
\hline Magnesium $(\mathrm{Mg})$........... & 5.2 & 14 & 11 & 11 & 24 \\
\hline Sodium (Na) .............. & 50 & 33 & 13 & 19 & 18 \\
\hline Potassium (K) ............ & 1.7 & 1.8 & 3.0 & 1.5 & 2.1 \\
\hline Carbonate $\left(\mathrm{CO}_{3}\right)$ & - & - & - & - & -- \\
\hline Bicarbonate $\left(\mathrm{HCO}_{3}\right) \ldots \ldots$ & 156 & 187 & 201 & 182 & 204 \\
\hline Sulfate $\left(\mathrm{SO}_{4}\right) \ldots \ldots \ldots \ldots \ldots$ & 48 & 60 & 11 & 21 & 121 \\
\hline Chloride (Cl) ............. & 13 & 15 & 7.0 & 9.3 & 14 \\
\hline Fluoride $(F)$ & 1,0 & .8 & .4 & .9 & .8 \\
\hline Nitrate $\left(\mathrm{NO}_{3}\right) \ldots \ldots \ldots \ldots \ldots$ & 5.6 & 7.4 & 13 & 2.5 & 28 \\
\hline Dissolved solids .......... & $\mathrm{b}_{238}$ & $b_{80}$ & 234 & $\mathrm{~b}_{208}$ & $b_{401}$ \\
\hline Hardness as $\mathrm{CaCO}_{3}$ : & & & & & \\
\hline 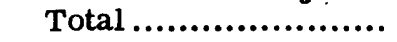 & 86 & 167 & 164 & 143 & 296 \\
\hline Noncarbonate ............ & 0 & 14 & 0 & 0 & 128 \\
\hline Color .... & -- & -- & -- & --1 & -- \\
\hline pH $\ldots . . . . . . .$. & 7.5 & 7.5 & 7.6 & 7.8 & 7.4 \\
\hline Specific conductance & & & 05 & & \\
\hline (micromhos at $25 \mathrm{C}$. ) & -- & -- & 355 & -- & -- \\
\hline Turbidity ..................... & -- & -- & 1.1 & -- & -- \\
\hline $\begin{array}{l}\text { Temperature }\left(F_{.}\right) \ldots . . . . . \\
\text { Date of collection ......... }\end{array}$ & Feb. 7 , & Feb. 7 & May 8 , & Feb. 7 & Feb. 7, \\
\hline & 1951 & 1951 & 1951 & 1951 & 1951 \\
\hline Depth (feet) & 462 & 466 & 550 & 714 & - \\
\hline Diameter (inches). & 22 & 16 & 26 & 20 & - \\
\hline Date drilled .. & 1921 & 1921 & 1927 & 1949 & -- \\
\hline Percent of supply ......... & -- & -- & -- & --1 & -- \\
\hline
\end{tabular}

Analyzed by California State Dept. of Public Health.

b Sum of determined constituents. 


\section{CALIFORNIA}

\section{BAKERSFIELD}

(Population, 34, 784)

Ownership: California Water Service Co. ; supplies East Bakersfield and other consumers outside the city limits. Total population supplied, about 75,000 .

Source: About 80 wells ranging in depth from 60 to $682 \mathrm{ft}$. The yield of the wells (data on 44 wells) is reported to range from 160 to $1,250 \mathrm{gpm}$, and to average 716 gal.

Treatment: None.

Storage: Reservoirs, 10, 300, 000 gal.

\section{ANALYSES}

(Analyses, in parts per million, by California Water Service Co., San Jose, Calif.)

\begin{tabular}{|c|c|c|c|c|c|}
\hline & & & & & $\begin{array}{c}\text { Range of } \\
\text { constituents }\end{array}$ \\
\hline 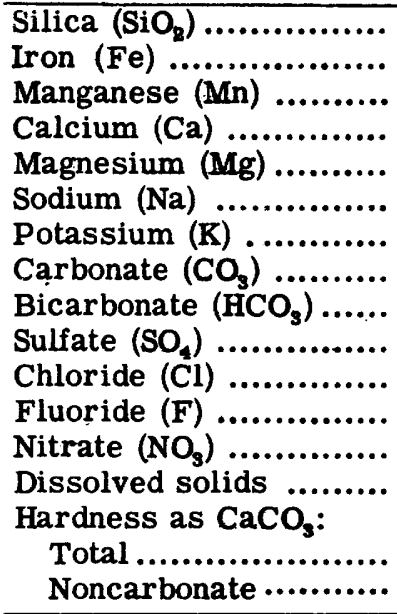 & 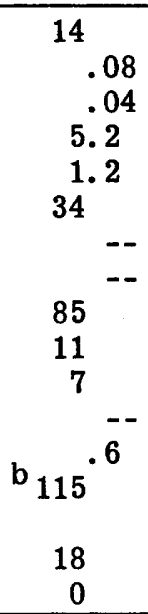 & \begin{tabular}{c}
20 \\
.01 \\
.06 \\
23 \\
3.9 \\
25 \\
\multicolumn{2}{c}{--} \\
115 \\
16 \\
10 \\
\multicolumn{2}{c}{--} \\
3.1 \\
157 \\
73 \\
0
\end{tabular} & 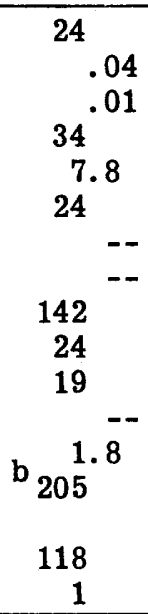 & $\begin{array}{r}205 \\
59 \\
38 \\
\\
\text { b } 16 \\
348 \\
214 \\
44\end{array}$ & 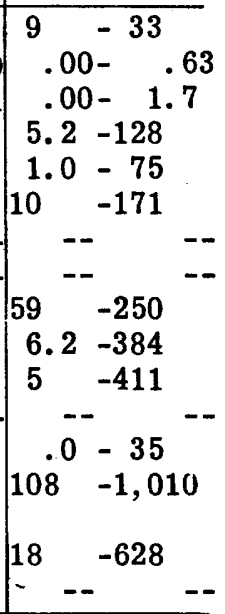 \\
\hline $\begin{array}{l}\text { Color } \\
\text { pH ... } \\
\text { Specif } \\
\text { (mi } \\
\text { Turbi } \\
\text { Temp } \\
\text { Date c }\end{array}$ & $\begin{array}{r}7.0 \\
180 \\
-- \\
-- \\
\text { Oct. } 27, \\
1950 \\
\end{array}$ & $\begin{array}{l}253 \\
\text { Sept. } \\
1950 \\
-\overline{1}\end{array}$ & $\begin{array}{r}339 \\
-- \\
-- \\
\text { Apr. } 23, \\
1951\end{array}$ & $\begin{array}{r}572 \\
- \\
\text { Apr. } 23, \\
1951\end{array}$ & $\begin{array}{rr}6.6 & 8.3 \\
180 & 1,620 \\
-- & -- \\
-\overline{1} & -\overline{1} \\
1948 & -1951\end{array}$ \\
\hline ..... & $\begin{array}{r}615 \\
16 \\
1949 \\
--\end{array}$ & $\begin{array}{r}300 \\
16 \\
1948 \\
--\end{array}$ & $\begin{array}{r}250 \\
16 \\
1946 \\
--\end{array}$ & $\begin{array}{r}240 \\
16 \\
1939 \\
--\end{array}$ & $\begin{array}{rr}60 & -682 \\
-- & -- \\
-- & -- \\
-- & --\end{array}$ \\
\hline
\end{tabular}

a Based on 80 analyses (1 analysis from each well) made between 1948-1951.

${ }^{b}$ Sum of determined constituents.

\section{BERKELEY}

(Population, 113, 805)

Ownership: East Bay Municipal Utility District. (See Oakland.) 


\title{
CALIFORNIA
}

\author{
BEVERLY HILLS \\ (Population, 29, 032)
}

Ownership: Municipal; supplies also West Hollywood. Total population supplied, about 41,000 .

Source: 17 wells ranging in depth from 90 to $702 \mathrm{ft}$ (83 percent of supply); Colorado River (17 percent of supply), furnished by the Metropolitan Water District of Southern California (see Los Angeles). The yield of the wells is reported to range from 100 to $900 \mathrm{gpm}$, and to average $540 \mathrm{gal}$.

Treatment: Aeration, softening with excess lime, recarbonation, rapid sand filtration, chlorination, and ammoniation.

Rated capacity of treatment plant: $7,500,000$ gpd.

Raw-water storage: 1, 171, 000 gal.

Finished-water storage: $18,780,000$ gal.

\section{ANALYSES}

(Analyses, in parts per million, by City of Beverly Hills)

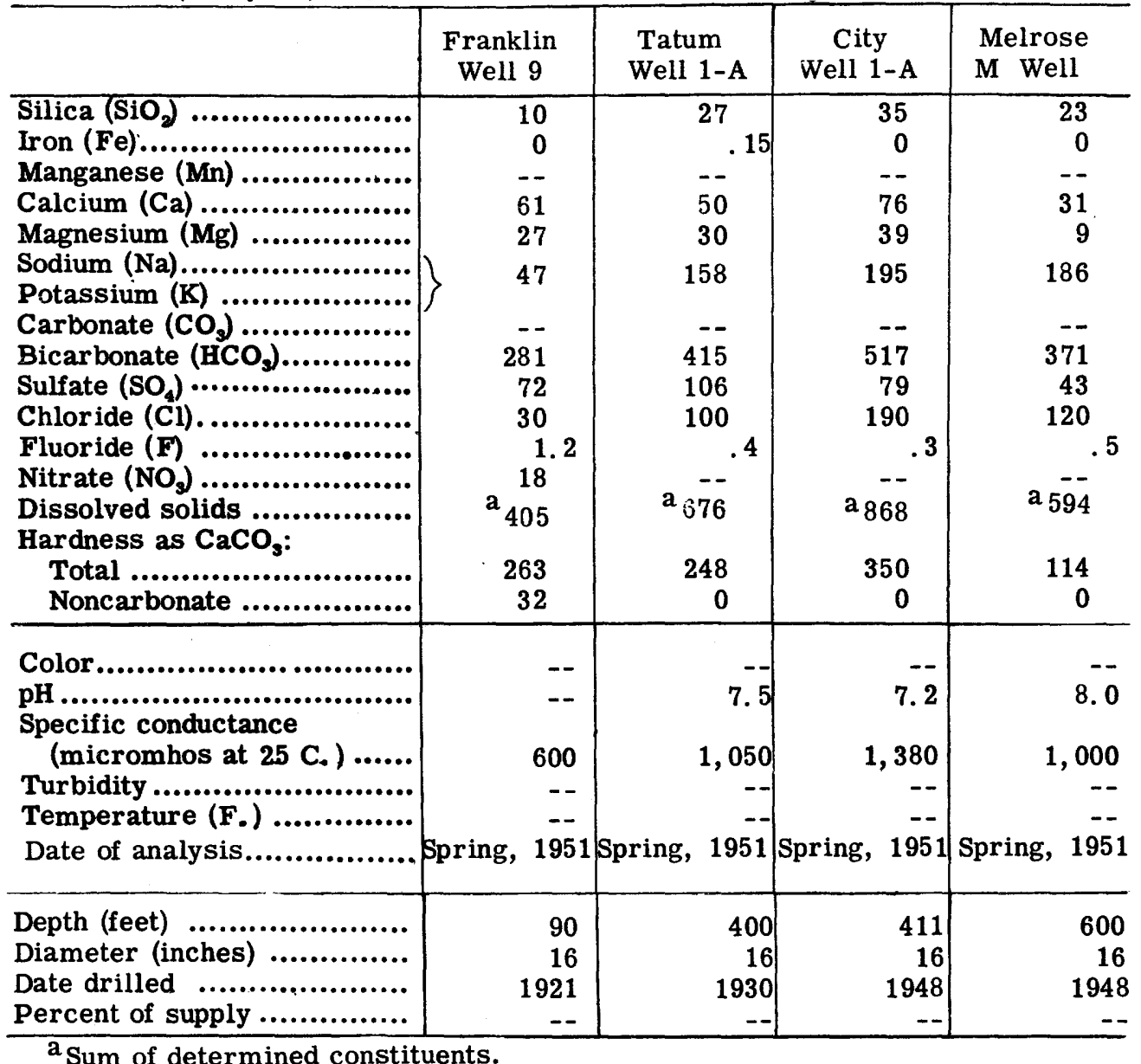




\section{CALIFORNIA}

BEVERLY HILLS--Continued

\section{ANALYSES}

(Analyses, in parts per million, by City of Beverly Hills)

\begin{tabular}{|c|c|c|c|c|}
\hline & $\begin{array}{c}\text { Plant } 1 \\
\text { (raw water) }\end{array}$ & $\begin{array}{c}\text { Plant } 1 \\
\text { (finished } \\
\text { water) }\end{array}$ & $\begin{array}{c}\text { Plant } 2 \\
\text { (raw water) }\end{array}$ & $\begin{array}{c}\text { Plant 2 } \\
\text { (finished } \\
\text { water) }\end{array}$ \\
\hline Silica (SiO, ...... & 20 & 20 & 12 & 12 \\
\hline Iron $(\mathrm{Fe}) \ldots \ldots \ldots \ldots \ldots \ldots \ldots$ & .20 & .10 & .20 & .20 \\
\hline Manganese (Mn) ................ & & & & -- \\
\hline Calcium (Ca) ........ & 75 & 25 & 42 & 22 \\
\hline Magnesium (Mg) & 30 & 26 & 16 & 12 \\
\hline 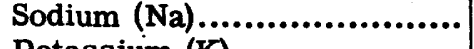 & 148 & 148. & 160 & 159 \\
\hline $\begin{array}{l}\text { Potassium }(\mathbf{K}) \ldots \ldots \ldots \ldots \ldots \ldots \\
\text { Carbonate }(\mathbf{C O}, \ldots \ldots \ldots \ldots \ldots \ldots \ldots\end{array}$ & 170 & 170. & 100 & 100 \\
\hline Bicarbonate $\left(\mathrm{HCO}_{3}\right) . . . \ldots \ldots \ldots . . .$. & 398 & 207 & 403 & 303 \\
\hline Sulfate $\left(\mathrm{SO}_{4}\right) \ldots \ldots \ldots \ldots \ldots \ldots$ & 108 & 110 & 68 & 70 \\
\hline Chloride $(\mathrm{Cl}), \ldots \ldots \ldots \ldots \ldots \ldots \ldots$ & 138 & 148 & 84 & 90 \\
\hline Fluoride (F) $\ldots \ldots \ldots \ldots \ldots \ldots$ & .4 & .4 & 6 & .6 \\
\hline Nitrate $\left(\mathrm{NO}_{3}\right), \ldots . . . . .$. & & & & -- \\
\hline Dissolved solids ..... & $a_{716}$ & ${ }^{a} 580$ & $a_{581}$ & a 515 \\
\hline Hardness as $\mathrm{CaCO}_{3}$ : & & & & \\
\hline Total . & 310 & 170 & 170 & 102 \\
\hline Noncarbonate .... & 0 & 0 & 0 & 0 \\
\hline Color.. & -- & -- & & -- \\
\hline pH ... & 7.5 & 8.2 & 8.0 & 8.1 \\
\hline Specific conductar & & & & 940 \\
\hline Turbidity .... & 1,100 & 920 & 20 & $\begin{array}{l}840 \\
--\end{array}$ \\
\hline Temperature (F.) .. & & & & \\
\hline Date of collect & Oct. 1951 & Oct. 1951 & Oct. 1951 & Oct. 1951 \\
\hline
\end{tabular}

Regular determinations at treatment plant, 1951

\begin{tabular}{|c|c|c|c|c|c|c|c|c|c|c|c|c|}
\hline & \multicolumn{3}{|c|}{$\begin{array}{c}\text { Alkalinity } \\
\text { as } \mathrm{CaCO} \\
\text { (ppm) }\end{array}$} & \multicolumn{3}{|c|}{ pH } & \multicolumn{3}{|c|}{$\begin{array}{c}\text { Hardness } \\
\text { as } \mathrm{CaCO} \\
(\mathrm{ppm})\end{array}$} & \multicolumn{3}{|c|}{ Turbidity } \\
\hline & $\mathbf{A v}$ & $\operatorname{Max}$ & Min & Av & $\operatorname{Max}$ & Min & Av & Max & Min & Av & $\operatorname{Max}$ & Min \\
\hline $\begin{array}{l}\text { Raw water.b....... } \\
\text { Finished water. }\end{array}$ & $=$ & $\begin{array}{l}330 \\
176\end{array}$ & $\begin{array}{l}300 \\
150\end{array}$ & $\begin{array}{l}-- \\
--\end{array}$ & $\begin{array}{l}-- \\
--\end{array}$ & $\begin{array}{l}7.6 \\
8.1\end{array}$ & $\begin{array}{l}-- \\
--\end{array}$ & $\begin{array}{l}320 \\
168\end{array}$ & $\begin{array}{l}300 \\
160\end{array}$ & -- & $\begin{array}{l}-- \\
--\end{array}$ & $\begin{array}{l}-- \\
--\end{array}$ \\
\hline $\begin{array}{l}\text { Raw water.c...... } \\
\text { Finished water... }\end{array}$ & -- & $\begin{array}{l}330 \\
250\end{array}$ & $\begin{array}{l}304 \\
230\end{array}$ & -- & -- & $\begin{array}{l}8.0 \\
8.1\end{array}$ & -- & $\begin{array}{l}172 \\
106\end{array}$ & $\begin{array}{r}164 \\
98\end{array}$ & -- & $\begin{array}{l}-- \\
--\end{array}$ & -- \\
\hline
\end{tabular}

${ }^{\text {a }}$ Sum of determined constituents.

b plant 1.

c plant 2. 


\section{CALIFORNIA}

\section{BURBANK}

(Population, 78, 577)

Ownership: Municipal.

Source: 11 wells $(3,4,6,7$, and 9 to 15$)$ ranging in depth from 180 to $790 \mathrm{ft}, 98$ percent of supply; Colorado River distributed by the Metropolitan Water District of Southern California, 2 percent of supply. (See Los Angeles.) The yield of the wells is reported to range from 500 to $2,860 \mathrm{gpm}$.

Treatment: The well water is not treated. Colorado River water is softened and filtered by the Metropolitan Water District of Southern California.

Raw-water storage: Approximately 35,000,000 gal.

Finished-water storage: --

\section{ANALYSES}

(Analyses, in parts per million, by Carl Wilson, Los Angeles)

\begin{tabular}{|c|c|c|c|c|c|}
\hline & Well 4 & Well 7 & Well 10 & Well 11 & Well 14 \\
\hline 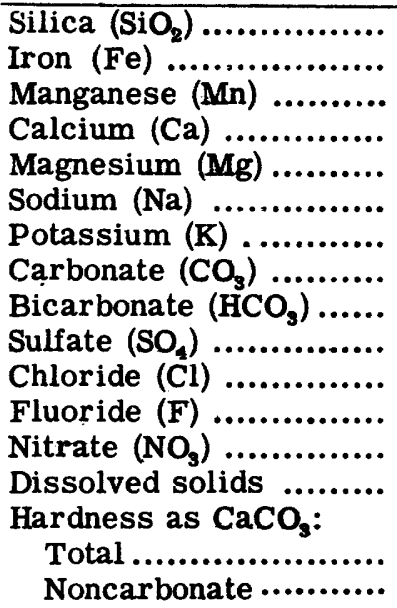 & $\begin{array}{r}20 \\
0 \\
0 \\
67 \\
18 \\
33 \\
-- \\
0 \\
275 \\
43 \\
24 \\
-- \\
13 \\
a_{354} \\
\\
241 \\
16\end{array}$ & $\begin{array}{r}19 \\
0 \\
0 \\
66 \\
17 \\
39 \\
-- \\
0 \\
275 \\
49 \\
25 \\
-- \\
12 \\
362 \\
\\
235 \\
10\end{array}$ & \begin{tabular}{r}
25 \\
0 \\
0 \\
52 \\
14 \\
35 \\
\multicolumn{2}{c}{--} \\
0 \\
242 \\
47 \\
12 \\
-- \\
a 305 \\
187 \\
0
\end{tabular} & $\begin{array}{r}24 \\
0 \\
0 \\
51 \\
15 \\
19 \\
-- \\
0 \\
189 \\
46 \\
16 \\
-- \\
5 \\
a_{269} \\
\\
189 \\
34\end{array}$ & $\begin{array}{r}19 \\
0 \\
0 \\
50 \\
14 \\
31 \\
-- \\
0 \\
222 \\
41 \\
16 \\
-- \\
4 \\
284 \\
182 \\
0\end{array}$ \\
\hline 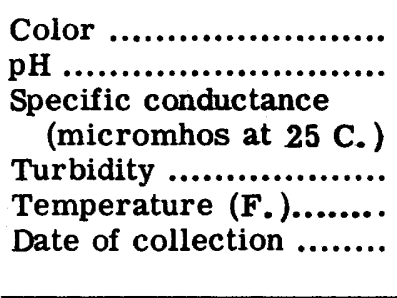 & $\begin{array}{r}-\overline{5} \\
7.5 \\
-- \\
-- \\
-- \\
\text { Sept. } \\
1951\end{array}$ & $\begin{array}{r}7.4 \\
-- \\
-- \\
-- \\
\text { Sept. } \\
1951\end{array}$ & $\begin{array}{r}7.9 \\
-- \\
-- \\
-- \\
\text { Sept. } \\
1951\end{array}$ & $\begin{array}{r}7.9 \\
-- \\
-- \\
-- \\
\text { Sept. } \\
1951\end{array}$ & $\begin{array}{r}7.8 \\
-- \\
-- \\
-- \\
\text { Sept. } \\
1951\end{array}$ \\
\hline $\begin{array}{l}\text { Depth (feet) } \ldots \ldots \ldots \ldots \ldots . . \\
\text { Diameter (inches)......... } \\
\text { Date drilled .............. } \\
\text { Percent of supply ........ }\end{array}$ & $\begin{array}{r}227 \\
16 \\
1924 \\
--\end{array}$ & $\begin{array}{r}630 \\
20 \\
1939 \\
-- \\
\end{array}$ & $\begin{array}{r}588 \\
20 \\
-- \\
--\end{array}$ & $\begin{array}{r}653 \\
20 \\
-- \\
--\end{array}$ & $\begin{array}{r}730 \\
20 \\
1950 \\
-- \\
\end{array}$ \\
\hline
\end{tabular}




\section{CALIFORNIA}

\section{COMPTON \\ (Population, 47, 991)}

Ownership: Municipal; supplies also about 1,000 people outside the city limits. Total population supplied, about 49,000 .

Source: 9 wells $(1,2,6$, and 8 to 13$), 256,282,280,717,642,466,640,410$, and $738 \mathrm{ft}$ deep. The yield of the wells is reported to be $630,790,360,740$, $1,200,1,080,2,000,450$, and $1,350 \mathrm{gpm}$. Emergency supply from the Colorado River distributed by the Metropolitan Water District of Southern California. (See Los Angeles.)

Treatment: None.

Storage: 3, 000, 000 gal.

\section{ANALYSES}

(Analyses, in parts per million, by California State Dept. of Public Health)

\begin{tabular}{|c|c|c|c|c|c|}
\hline & Well 1 & Well 6 & Well 9 & Well 10 & Well 11 \\
\hline 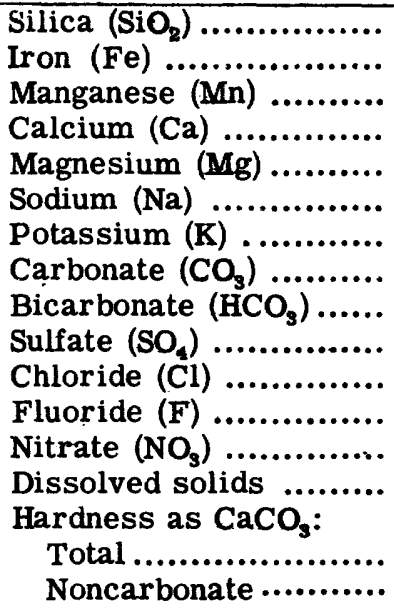 & $\begin{array}{c}.03 \\
.0 \\
57^{--} \\
12 \\
40 \\
\\
0 \\
212 \\
71 \\
22 \\
.4 \\
.0 \\
336 \\
191 \\
18\end{array}$ & 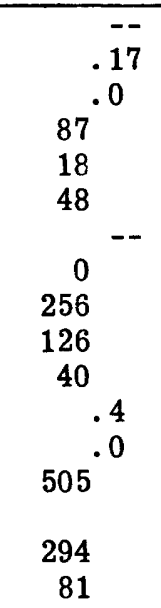 & 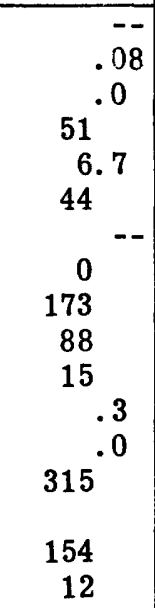 & \begin{tabular}{c}
.-- \\
.03 \\
68 \\
12 \\
27 \\
\multicolumn{2}{c}{-0} \\
0 \\
210 \\
65 \\
26 \\
.4 \\
.1 \\
387 \\
219 \\
47
\end{tabular} & $\begin{array}{l}.04 \\
.0 \\
58 \\
10 \\
43 \\
5^{-0} \\
5 \\
205 \\
71 \\
21 \\
.3 \\
.0 \\
400^{-0} \\
187 \\
10\end{array}$ \\
\hline 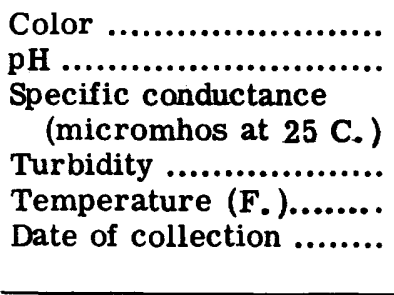 & $\begin{array}{r}7.9 \\
-- \\
0 \\
\text { Mar. } 21, \\
1951\end{array}$ & $\begin{array}{r}7.6 \\
-- \\
0 \\
-- \\
\text { Mar. } 21, \\
1951\end{array}$ & $\begin{array}{r}8.2 \\
-- \\
0 \\
\operatorname{Mar.} 21, \\
1951\end{array}$ & 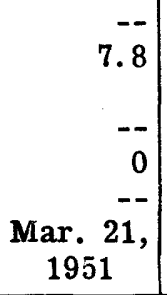 & $\begin{array}{c}8.3 \\
-- \\
-- \\
0 \\
-- \\
\text { Mar. } 21, \\
1951\end{array}$ \\
\hline $\begin{array}{l}\text { Depth (feet) } \ldots . \ldots \ldots \ldots . . . \\
\text { Diameter (inches)........ } \\
\text { Date drilled ............... } \\
\text { Percent of supply ........ }\end{array}$ & $\begin{array}{r}256 \\
12 \\
1920 \\
--\end{array}$ & $\begin{array}{r}280 \\
8 \\
1880 \\
--\end{array}$ & $\begin{array}{r}642 \\
28,16 \\
1947 \\
--\end{array}$ & $\begin{array}{r}466 \\
16 \\
1947 \\
--\end{array}$ & $\begin{array}{r}640 \\
16 \\
1948 \\
--\end{array}$ \\
\hline
\end{tabular}




\section{CALIFORNIA}

\section{CONTRA COSTA DISTRICT \\ (Population, 43, 000)}

Ownership: California Water Service Co. ; supplies Concord, Crockett, Mountain View, Port Chicago, and San Ramon Valley. Total population supplied, about 43,000 .

Source: San Joaquin River through the Contra Costa Canal (47 percent of supply), Sacramento River ( 45 percent of supply), Port Chicago wells (5 percent of supply), and other wells (3 percent of supply). There is an emergency connection with the East Bay Municipal Utility District. (See Oakland.)

Treatment: Surface waters: prechlorination, coagulation with alum, sedimentation, activated carbon, rapid sand filtration, postchlorination, and adjustment of $\mathrm{pH}$ with soda ash or lime. Water from the Galindo wells is softened with zeolite.

Rated capacity of treatment plant: 6, 000, 000 gpd.

Raw-water storage: 1, 000, 000, $000 \mathrm{gal}$.

Finished-water storage: $5,200,000 \mathrm{gal}$.

\section{ANALYSES}

(Analyses, in parts per million, by California Water Service Co., San Jose, Calif.)

\begin{tabular}{|c|c|c|c|c|}
\hline & $\begin{array}{c}\text { Galindo } \\
\text { wells } \\
\text { (raw water) } \\
\end{array}$ & $\begin{array}{c}\text { Government } \\
\text { Ranch } \\
\text { wells }\end{array}$ & \begin{tabular}{|c|} 
Mallard \\
Reservoir \\
(raw water)
\end{tabular} & $\begin{array}{r}\text { Finished } \\
\text { water b }\end{array}$ \\
\hline Silica (SiO) $\ldots . .$. & 25 & 21 & 8 & $\overline{8}$ \\
\hline Iron $(\mathrm{Fe}) . . . . . . . . . . . .$. & .06 & .15 & .93 & .10 \\
\hline Manganese (Mn) ...... & .10 & .18 & .13 & .11 \\
\hline Calcium (Ca) ......... & 46 & 41 & & \\
\hline Magnesium $(\mathrm{Mg})$................ & 36 & 31 & 22 & 19 \\
\hline Sodium $(\mathrm{Na}) \ldots \ldots \ldots \ldots \ldots \ldots \ldots \ldots$ & 41 & 119 & 65 & 56 \\
\hline 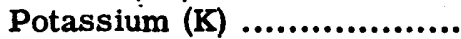 & -- & -- & -- & -- \\
\hline Carbonate $\left(\mathrm{CO}_{3}\right) \ldots . .$. & -- & & - & -- \\
\hline Bicarbonate $\left(\mathrm{HCO}_{3}\right) \ldots \ldots \ldots \ldots \ldots$ & 279 & 288 & 107 & 112 \\
\hline Sulfate $\left(\mathrm{SO}_{4}\right) \ldots \ldots \ldots \ldots \ldots \ldots$ & 56 & 79 & 60 & 77 \\
\hline Chloride $(\mathrm{Cl}), \ldots \ldots \ldots \ldots \ldots \ldots \ldots$ & 38 & 113 & 103 & 70 \\
\hline Fluoride $(F) \quad \ldots \ldots \ldots \ldots \ldots$ & 1 & .1 & 0 & .0 \\
\hline Nitrate $\left(\mathrm{NO}_{3}\right) \ldots \ldots \ldots \ldots \ldots \ldots \ldots$ & 11 & 11 & .6 & 3.7 \\
\hline Dissolved solids ........ & $c_{391}$ & $c_{557}$ & $c_{338}$ & \\
\hline Hardness as $\mathrm{CaCO}_{3}$ : & & & & \\
\hline Total ............... & 262 & 230 & 155 & 152 \\
\hline Nonc & 34 & 0 & 68 & 61 \\
\hline Color & -- & -- & 0 & -- \\
\hline pH........ & 7.9 & 7.5 & 7.2 & 8.0 \\
\hline Specific conductance & & & & \\
\hline (micromhos at 25 C. ) ....... & -- & -- & -- & -- \\
\hline Turbidity ................. & - & & -- & -- \\
\hline re (F.).. & -- & & & \\
\hline Date of collection.... & $\begin{array}{c}\text { Feb. } 8, \\
1950\end{array}$ & $\begin{array}{c}\text { Jan. 23, } \\
1950\end{array}$ & $\begin{array}{c}\text { Jan. 3, } \\
1950\end{array}$ & 1949 \\
\hline
\end{tabular}

a Surface waters.

${ }^{b}$ Composite sample, distribution system.

c Sum of determined constituents. 


\section{CA LIFORNIA}

CONTRA COSTA DISTRICT --Continued

\begin{tabular}{|c|c|c|c|c|}
\hline & $\begin{array}{c}\text { Galindo } \\
\text { wells } \\
\text { (raw water) }\end{array}$ & $\begin{array}{c}\text { Government } \\
\text { Ranch } \\
\text { wells }\end{array}$ & $\begin{array}{c}\text { Mallard } \\
\text { Reservoir } \\
\text { (raw water) }\end{array}$ & $\begin{array}{c}\text { Finished } \\
\text { water }\end{array}$ \\
\hline 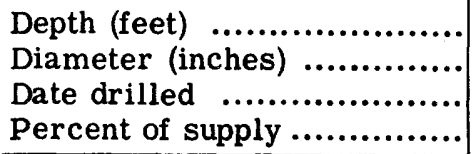 & $\begin{array}{r}193-498 \\
10-12 \\
1910-1929 \\
--\end{array}$ & $\begin{array}{r}114-610 \\
12 \\
1925-1928 \\
--\end{array}$ & & \\
\hline
\end{tabular}

Regular determinations at treatment plant, 1950

\begin{tabular}{l|r|r|r|r|r|r|r|r|r|r|r|r|}
\hline & \multicolumn{3}{|c|}{$\begin{array}{c}\text { Alkalinity } \\
\text { as CaCO } \\
\text { (ppm) }\end{array}$} & \multicolumn{3}{c|}{ pH } & \multicolumn{3}{c|}{$\begin{array}{c}\text { Hardness } \\
\text { as CaCO } \\
\text { (ppm) }\end{array}$} & \multicolumn{3}{c}{ Turbidity } \\
\cline { 2 - 13 } & Av & Max & Min & Av & Max & Min & Av & Max & Min & Av & Max & Min \\
\hline Raw water......... & -- & 238 & 88 & 7.5 & 7.9 & 7.2 & 155 & 262 & 152 & -- & -- & -- \\
Finished water... & 92 & -- & -- & 8.0 & -- & -- & 152 & -- & -- & -- & -- & -- \\
\hline
\end{tabular}




\title{
CALIFORNIA
}

\author{
CULVER CITY \\ (Population, 19, 720)
}

Ownership: Southern California Water Co. ; supplies also about 4, 100 people outside the city limits. Total population supplied, about 23,800 .

Source: 10 wells. Sentney Plant: wells (5 to 8, 10, and 12), 266, 810, 287, 320, 290, and $650 \mathrm{ft}$ deep; Pacific Plant: well (4), $335 \mathrm{ft}$ deep; Sepulveda Plant: well (3), $300 \mathrm{ft}$ deep; Manning Plant: wells (4 and 5), 304 and $855 \mathrm{ft}$ deep. The yield of the wells is reported to range from 135 to $1,150 \mathrm{gpm}$.

Treatment: Aeration, coagulation with ferrous chloride, activated carbon, sedimentation, rapid sand filtration, and chlorination.

Rated capacity of treatment plant: $2,500,000 \mathrm{gpd}$.

Raw-water storage: None.

Finished-water storage: 1,350, $000 \mathrm{gal}$.

\section{ANALYSES}

(Analyses, in parts per million, by Smith-Emery Co., Los Angeles, Calif.)

\begin{tabular}{|c|c|c|c|c|}
\hline & $\begin{array}{c}\text { Sentney } \\
\text { Wells }\end{array}$ & $\begin{array}{c}\text { Pacific } \\
\text { Well } \\
\end{array}$ & $\begin{array}{l}\text { Sepulveda } \\
\text { Well }\end{array}$ & $\begin{array}{c}\text { Manning } \\
\text { Wells }\end{array}$ \\
\hline 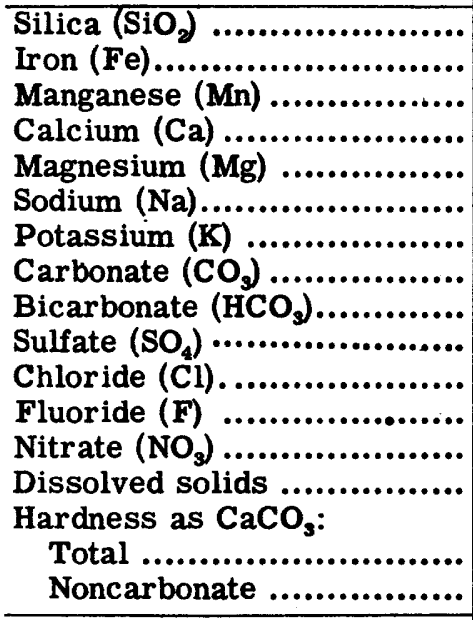 & 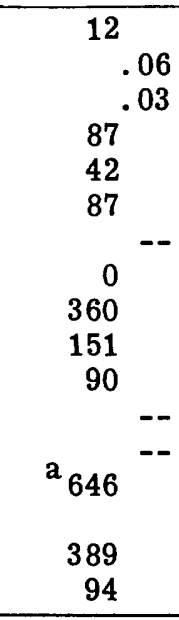 & 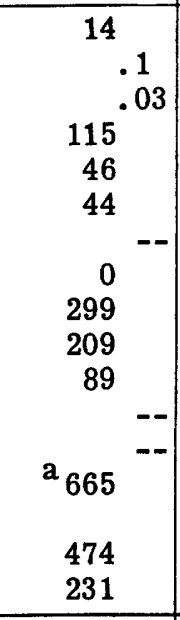 & 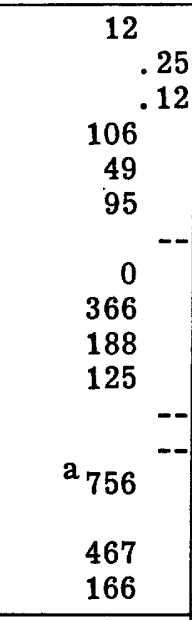 & \begin{tabular}{c}
12 \\
.04 \\
87 \\
30 \\
30 \\
46 \\
\multicolumn{2}{c}{-00} \\
0 \\
337 \\
97 \\
43 \\
\\
\\
$a_{481}^{--}$ \\
\\
340 \\
64
\end{tabular} \\
\hline 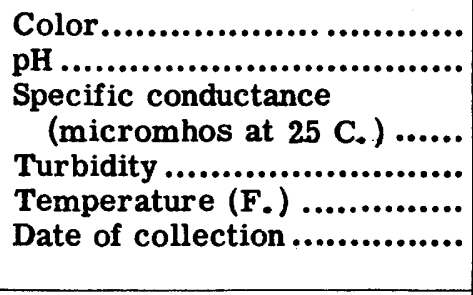 & $\begin{array}{r}8.0 \\
-- \\
-- \\
\text { Mar. } 27, \\
1950\end{array}$ & $\begin{array}{r}7.8 \\
-- \\
-- \\
-- \\
\text { Apr. } 7, \\
1950\end{array}$ & $\begin{array}{c}\text { Apr. } \\
1950 \\
\end{array}$ & $\begin{array}{r}7.6 \\
-- \\
-- \\
\text { June } 2 \text {,- } \\
1950 \\
\end{array}$ \\
\hline 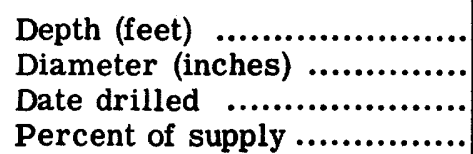 & $\begin{array}{r}266-810 \\
14-18 \\
1931-1946 \\
--\end{array}$ & $\begin{array}{r}335 \\
16 \\
1932 \\
--\end{array}$ & $\begin{array}{r}300 \\
16 \\
1938 \\
--\end{array}$ & $\begin{array}{r}304-855 \\
10-14 \\
1938-1944 \\
--\end{array}$ \\
\hline
\end{tabular}




\section{CALIFORNIA}

\section{EAST BAKERSFIELD}

(Population, 38, 177)

Ownership: California Water Service Co. (See Bakersfield.)

\section{EAST LOS ANGELES}

(Population, 85, 900)

Ownership: California Water Service Co.

Source: 43 wells ranging in depth from 276 to $815 \mathrm{ft}$. The depth of most of the wells is around $500 \mathrm{ft}$.

Treatment: Chlorination.

Raw-water storage: None.

Finished-water storage: $8,140,000$ gal.

\section{ANALYSIS}

(Analysis, in parts per million, by California Water Service Co., San Jose, Calif.)

\begin{tabular}{|c|c|c|c|}
\hline & $\begin{array}{l}\text { Typical } \\
\text { composite } \\
\text { of wells }\end{array}$ & & $\begin{array}{l}\text { Typical } \\
\text { composite } \\
\text { of wells }\end{array}$ \\
\hline \multirow{2}{*}{ 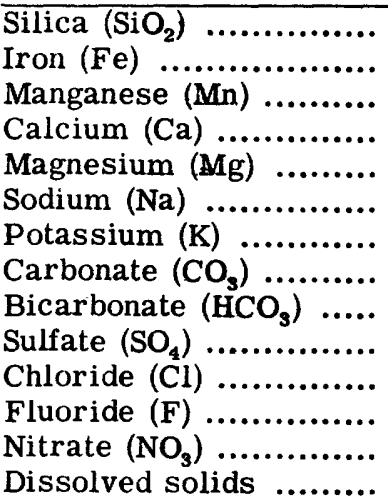 } & $\begin{array}{l}28 \\
.04 \\
57 \\
57 \\
17 \\
37\end{array}$ & $\begin{array}{l}\text { Hardness as } \mathrm{CaCO}_{\mathbf{3}}: \\
\quad \text { Total ...................... } \\
\text { Noncarbonate ........... }\end{array}$ & $\begin{array}{r}212 \\
43\end{array}$ \\
\hline & \begin{tabular}{r}
57 \\
17 \\
37 \\
\\
0 \\
206 \\
55 \\
46 \\
\multicolumn{2}{c}{.0} \\
$a_{342} .0$
\end{tabular} & 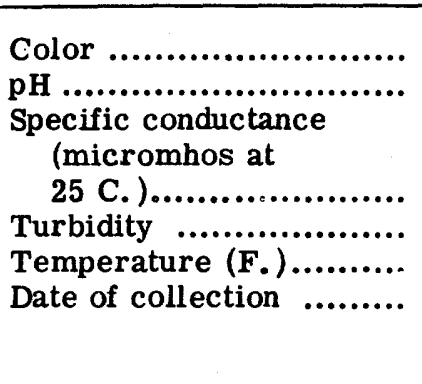 & $\begin{array}{r}-- \\
-- \\
1950\end{array}$ \\
\hline \multicolumn{3}{|c|}{ 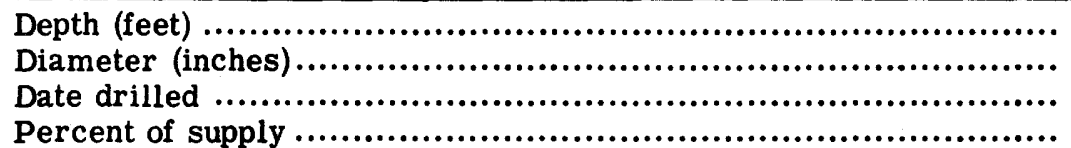 } & $\overline{--}$ \\
\hline
\end{tabular}

\footnotetext{
${ }^{\mathrm{a}}$ Sum of determined constituents.
} 


\section{CALIFORNIA}

\section{EUREKA}

(Population, 23, 058)

Ownership: Municipal; supplies also about 8,000 people outside the city limits.

Total population supplied, about 31,000.

Source: Mad River (impounded); emergency supply from 3 deep wells.

Treatment: Prechlorination; coagulation with alum, sodium aluminate, and lime; rapid sand filtration; activated carbon for odor and taste control; postchlorination and ammoniation in the transmission main.

Rated capacity of treatment plant: 2, 500, 000 gpd.

Raw-water storage: Approximately 750,000,000 gal impounded behind Mad River Dam.

Finished-water storage: 2, 125, 000 gal.

\section{ANALYSES}

(Analyses, in parts per million, by California State Dept. of Public Health)

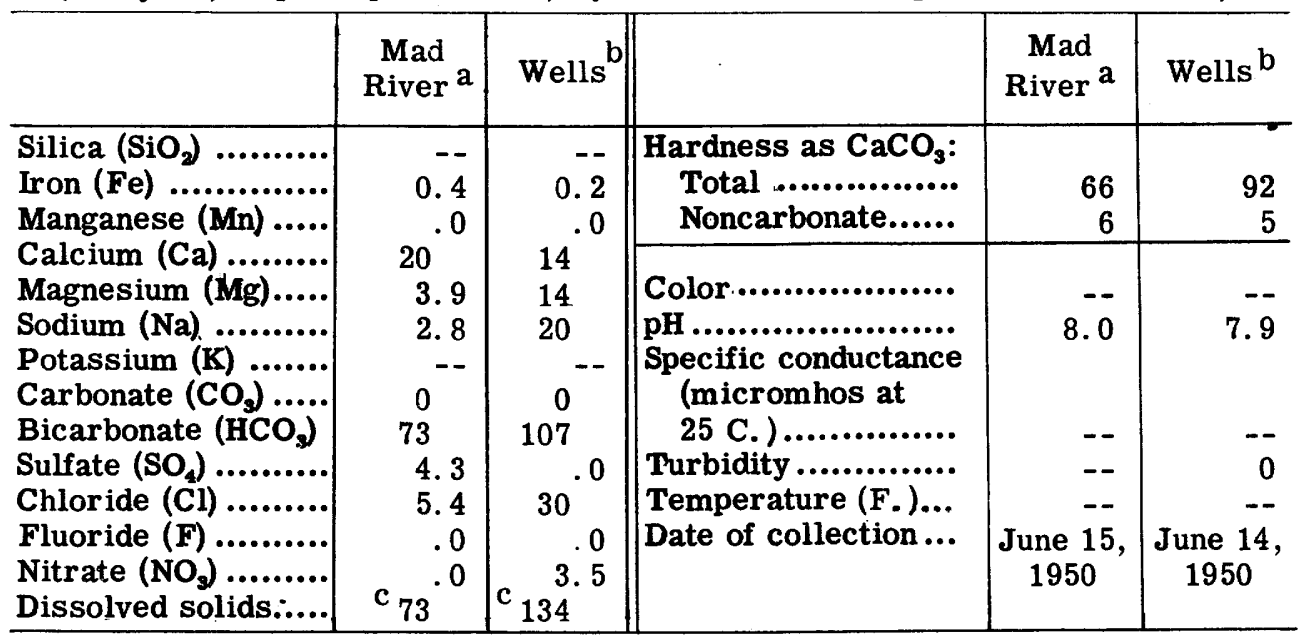

Regular determinations at treatment plant, 1950

\begin{tabular}{l|r|r|r|r|r|c|c|c|c|c|c|c}
\hline & \multicolumn{3}{|c|}{$\begin{array}{c}\text { Alkalinity } \\
\text { as CaCO } \\
\text { (ppm) }\end{array}$} & \multicolumn{3}{c|}{$\mathrm{pH}$} & \multicolumn{3}{c|}{$\begin{array}{c}\text { Hardness } \\
\text { as CaCO } \\
\text { (ppm) }\end{array}$} & \multicolumn{3}{c}{ Turbidity } \\
\cline { 2 - 13 } & Av & Max & Min & Av & Max & Min & Av & Max & Min & Av & Max & Min \\
\hline Raw water......... & 50 & 100 & 20 & 7.2 & 7.5 & 6.5 & 50 & 100 & 30 & & 71,500 & 5 \\
Finished water... & 40 & 90 & 8 & 7.0 & 7.3 & 6.8 & 60 & 100 & 35 & & 50 & 5 \\
\hline
\end{tabular}

a Finished water except for final chlorination.

${ }^{b}$ Hawthorne Street wells.

c Sum of determined constituents. 


\title{
CALIFORNIA
}

\author{
FRESNO \\ (Population, 91, 669)
}

Ownership: Municipal; supplies also about 16,000 people outside the city limits.

Total population supplied, about 108, 000 .

Source: 45 wells ranging in depth from 80 to $319 \mathrm{ft}$. The depths of most of the wells are between 100 and $200 \mathrm{ft}$. The yield of the wells is reported to range from 1,000 to $2,425 \mathrm{gpm}$, and to average 1,489 .

Treatment: None.

Storage: $1,880,000$ gal.

\section{ANALYSES}

(Analyses, in parts per million, by U. S. Geological Survey)

\begin{tabular}{|c|c|c|c|c|c|}
\hline & Well 2 & Well 3 & Well 24 & Well 27 & Well $32^{a}$ \\
\hline 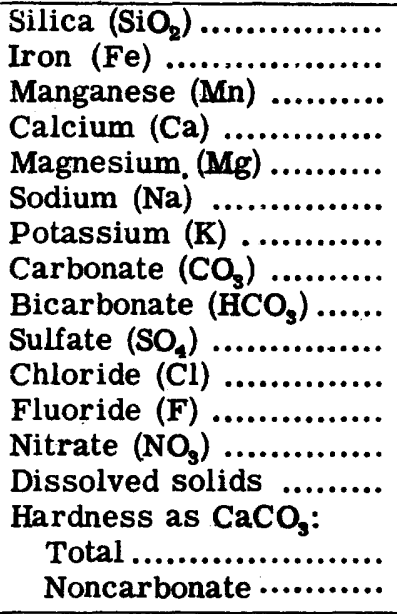 & $\begin{array}{c}3 \\
.02 \\
34 \\
19 \\
29 \\
5.9 \\
0 \\
202 \\
13 \\
20 \\
.0 \\
27 \\
330 \\
163 \\
0 \\
\end{array}$ & $\begin{array}{c}80 \\
.01 \\
28 \\
17 \\
23 \\
5.2 \\
0 \\
172 \\
9.3 \\
18 \\
.0 \\
22 \\
286 \\
140 \\
0 \\
\end{array}$ & $\begin{array}{c}71 \\
.0 \\
15 \\
8.3 \\
18 \\
3.4 \\
0 \\
101 \\
9.6 \\
6.8 \\
.0 \\
14 \\
202 \\
72 \\
0 \\
\end{array}$ & $\begin{array}{c}73 \\
.0 \\
18 \\
13 \\
16 \\
4.4 \\
0 \\
130 \\
8.4 \\
8.0 \\
.0 \\
14 \\
217 \\
98 \\
0 \\
\end{array}$ & $\begin{array}{c}58 \\
.01 \\
.00 \\
25 \\
14 \\
22 \\
4.3 \\
0 \\
174 \\
3.3 \\
18 \\
.1 \\
5.4 \\
236 \\
120 \\
0 \\
\end{array}$ \\
\hline 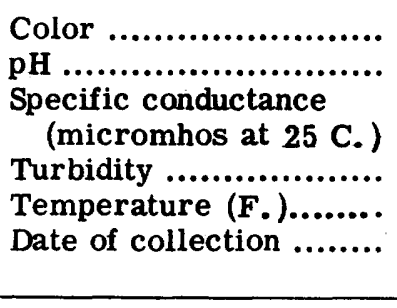 & $\begin{array}{r}7.8 \\
451 \\
0 \\
-- \\
\text { Oct. } 17, \\
1951 \\
\end{array}$ & $\begin{array}{r}5 \\
7.6 \\
378 \\
1 \\
-\overline{17} \\
\text { Oct. } 17 \\
1951 \\
\end{array}$ & $\begin{array}{r}10 \\
7.7 \\
231 \\
3 \\
-- \\
\text { Oct. } 17 \\
1951 \\
\end{array}$ & $\begin{array}{r}3.6 \\
269 \\
0 \\
-- \\
\text { Oct. } 17, \\
1951 \\
\end{array}$ & $\begin{array}{c}-- \\
\text { May 2, } \\
1947\end{array}$ \\
\hline $\begin{array}{l}\text { Depth (feet) } \ldots . . . \ldots \ldots . . . . \\
\text { Diameter (inches)........ } \\
\text { Date drilled ............... } \\
\text { Percent of supply ........ }\end{array}$ & $\begin{array}{r}142 \\
20 \\
1922 \\
--\end{array}$ & $\begin{array}{r}123 \\
18 \\
1923 \\
--\end{array}$ & $\begin{array}{r}162 \\
18 \\
1925 \\
--\end{array}$ & $\begin{array}{r}130 \\
18 \\
-- \\
--\end{array}$ & $\begin{array}{r}182 \\
20 \\
1941 \\
--\end{array}$ \\
\hline
\end{tabular}

${ }^{\mathrm{a}}$ Analyzed by Twining Laboratories, Fresno, Calif. 


\section{CALIFORNIA}

\section{GLENDALE}

(Population, 95, 702)

Ownership: Municipal; supplies also about 400 people outside the city limits. Total population supplied, about 96,100 .

Source: 11 wells (Grandview 1 to $4,6,9$ to 12, and Glorietta 3 and 4) ranging in depth from 180 to $640 \mathrm{ft}$ and Verdugo Stream, about 99 percent of supply. The yield of the wells is reported to range from 835 to $3,190 \mathrm{gpm}$, and averages $2,117 \mathrm{gpm}$. Most of the supply is from the wells. Auxiliary supply (about 1 percent of the total), Colorado River distributed by the Metropolitan Water District of Southern California. (See Los Angeles.)

Treatment: None (Colorado River water is softened and filtered by Metropolitan Water District of Southern California). (See Los Angeles.)

Storage: $170,000,000$ gal.

\section{ANALYSES}

(Analyses, in parts per million, by California State Dept. of Public Health)

\begin{tabular}{|c|c|c|c|c|c|}
\hline & $\begin{array}{c}\text { Grandview } \\
\text { Well } 6\end{array}$ & $\begin{array}{c}\text { Grandview } \\
\text { Well } 11\end{array}$ & $\mid \begin{array}{c}\text { Grandview } \\
\text { Wells }^{\mathrm{a}}\end{array}$ & $\begin{array}{c}\text { Glorietta } \\
\text { Well } 3\end{array}$ & $\begin{array}{l}\text { Verdugo } \\
\text { Stream }\end{array}$ \\
\hline 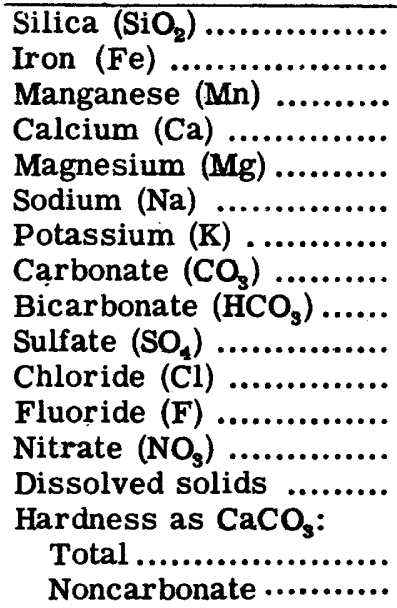 & 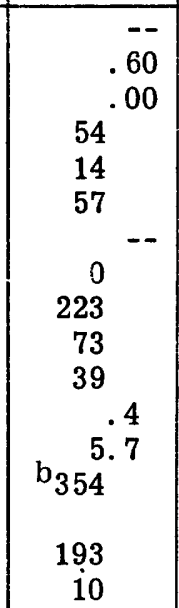 & 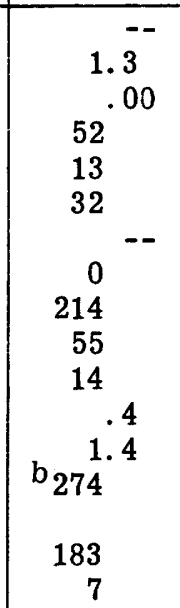 & \begin{tabular}{c|}
19 \\
0 \\
56 \\
12 \\
71 \\
$0^{--}$ \\
243 \\
67 \\
51 \\
-- \\
3.5 \\
$\mathrm{~b}_{399}$ \\
189 \\
0
\end{tabular} & $\begin{array}{c}3.8 \\
.00 \\
36 \\
14 \\
20 \\
0^{--} \\
149 \\
23 \\
14 \\
.4 \\
26 \\
\mathrm{~b}_{211} \\
150 \\
28\end{array}$ & 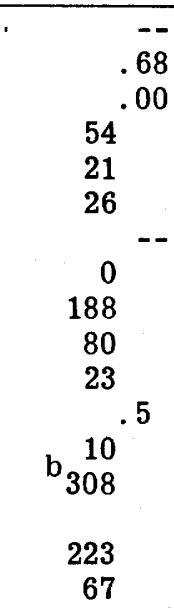 \\
\hline 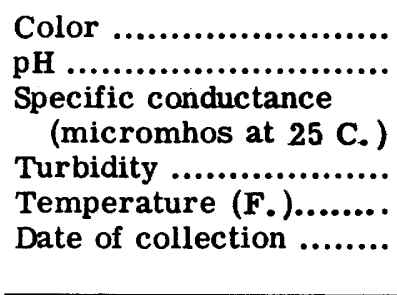 & $\begin{array}{r}7.7 \\
628 \\
-- \\
-- \\
\text { Oct. } 19 \\
1951\end{array}$ & $\begin{array}{r}491 \\
-- \\
\overline{-} \\
\text { Oct. } 19 \\
1951\end{array}$ & $\begin{array}{r}7.7 \\
-- \\
-- \\
\text { Nov. } 29, \\
1949\end{array}$ & $\begin{array}{r}389 \\
-- \\
-- \\
\text { Oct. } 19, \\
1951\end{array}$ & $\begin{array}{r}6.8 \\
545 \\
-- \\
\text { Oct. } 19, \\
1951\end{array}$ \\
\hline $\begin{array}{l}\text { Depth (feet) } \ldots \ldots \ldots \ldots \ldots . . \\
\text { Diameter (inches)......... } \\
\text { Date drilled ............... } \\
\text { Percent of supply ........ }\end{array}$ & $\begin{array}{r}476 \\
18 \\
1923 \\
--\end{array}$ & $\begin{array}{r}640 \\
18 \\
1929 \\
--\end{array}$ & $\begin{array}{l}-- \\
-- \\
-- \\
--\end{array}$ & $\begin{array}{r}180 \\
16 \\
1928 \\
--\end{array}$ & \\
\hline
\end{tabular}

${ }^{a}$ Analyzed by Carl Wilson, Los Angeles, Calif.

$b_{\text {Sum of determined constituents. }}$ 


\section{CALIFORNIA}

\section{HUNTINGTON PARK}

(Population, 29, 450)

Ownership: Municipal.

Source: 11 wells ranging in depth from 510 to $1,550 \mathrm{ft}$. The yield of the wells is reported to range from 200 to $1,440 \mathrm{gpm}$. Emergency supply from wells owned by Southern California Water Co. in Bell and Maywood.

Treatment: None.

Storage: $7,442,000$ gal.

The weighted average hardness and dissolved solids of the water served are 228 ppm and $365 \mathrm{ppm}$, respectively.

\section{ANALYSES}

(Analyses, in parts per million, by California State Dept. of Public Health)

\begin{tabular}{|c|c|c|c|c|c|}
\hline & Well 2 & Well 6 & Well 7 & Well 10 & Well 12 \\
\hline 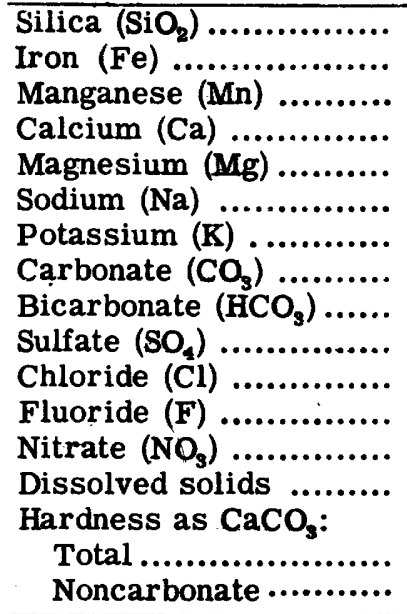 & \begin{tabular}{c}
15 \\
.03 \\
.00 \\
72 \\
26 \\
54 \\
1.9 \\
\multicolumn{2}{c|}{} \\
216 \\
121 \\
75 \\
.4 \\
7.1 \\
$a_{479}$
\end{tabular} & $\begin{array}{c}20 \\
.00 \\
.00 \\
85 \\
47 \\
60 \\
1.4 \\
\quad-- \\
248 \\
205 \\
89 \\
.4 \\
747^{.4}\end{array}$ & \begin{tabular}{c}
23 \\
.00 \\
.05 \\
48 \\
15 \\
42 \\
3.0 \\
\multicolumn{2}{c}{-0} \\
193 \\
76 \\
22 \\
.3 \\
349 \\
.8
\end{tabular} & \begin{tabular}{|c|}
14 \\
.00 \\
.00 \\
47 \\
20 \\
44 \\
2.4 \\
201 \\
201 \\
82 \\
24 \\
.4 \\
3.5 \\
373 \\
201 \\
35
\end{tabular} & $\begin{array}{c}17 \\
.04 \\
.00 \\
46^{\circ} \\
13 \\
45 \\
2.3 \\
204^{--} \\
69 \\
23 \\
.4 \\
.0 \\
345^{.0} \\
170 \\
3\end{array}$ \\
\hline 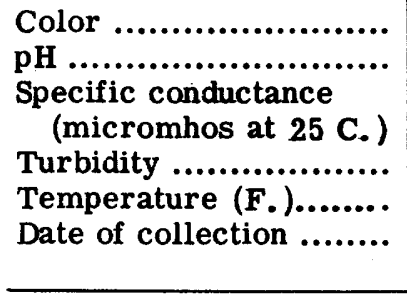 & $\begin{array}{r}7.4 \\
824 \\
<\quad 1 \\
-- \\
\text { Sept. } 25 \\
1951 \\
\end{array}$ & $\begin{array}{l}7.5 \\
1,000 \\
<\quad 1 \\
-- \\
\text { Sept. } 25 \\
1951\end{array}$ & $\begin{array}{r}7.7 \\
518 \\
<1 \\
-- \\
\text { Sept. } 26 \\
1951\end{array}$ & $\begin{array}{r}7.7 \\
548 \\
<1 \\
--- \\
\text { Sept. } 25 \\
1951\end{array}$ & $\begin{array}{r}7.8 \\
521 \\
<1 \\
\text { Sept. } 25, \\
1951\end{array}$ \\
\hline $\begin{array}{l}\text { Depth (feet) .............. } \\
\text { Diameter (inches)......... } \\
\text { Date drilled ............... } \\
\text { Percent of supply ........ }\end{array}$ & $\begin{array}{r}533 \\
12 \\
1920 \\
-- \\
\end{array}$ & $\begin{array}{r}756 \\
12 \\
1908 \\
-- \\
\end{array}$ & $\begin{array}{r}984 \\
12 \\
1909 \\
-- \\
\end{array}$ & $\begin{array}{r}1,200 \\
18 \\
1937 \\
-- \\
\end{array}$ & $\begin{array}{r}1,504 \\
14 \\
1945 \\
-- \\
\end{array}$ \\
\hline
\end{tabular}

${ }^{a}$ Sum of determined constituents. 


\section{CALIFORNIA}

\section{INGLEWOOD}

(Population, 46, 185)

Ownership: Municipal; supplies also about 100 people outside the city limits. Total population supplied, about 46,300 .

Source: About 25 wells ranging in depth from 282 to $798 \mathrm{ft}$. Most of the wells are under $500 \mathrm{ft}$ in depth.

Treatment: Chlorination.

Storage: $4,800,000$ gal.

\section{ANALYSES}

(Analyses, in parts per million, by Montgomery and Pomeroy, Los Angeles, Calif.)

\begin{tabular}{|c|c|c|c|c|}
\hline & $\begin{array}{c}\text { Wells } 9, \\
11,19,24\end{array}$ & $\begin{array}{l}\text { Well } \\
22\end{array}$ & $\begin{array}{c}\text { Well } \\
29\end{array}$ & $\begin{array}{c}\text { Well } \\
33\end{array}$ \\
\hline 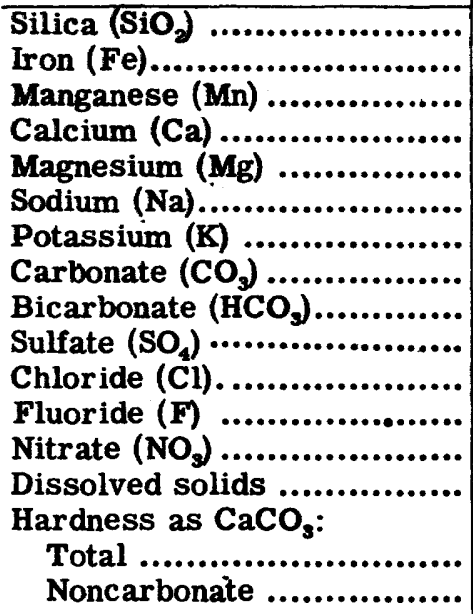 & \begin{tabular}{l}
19 \\
.0 \\
71 \\
14 \\
52 \\
\multicolumn{2}{c}{-0} \\
0 \\
249 \\
94 \\
32 \\
.0 \\
1.8 \\
406 \\
235 \\
31
\end{tabular} & $\begin{array}{c}19 \\
.0 \\
.0 \\
84 \\
24 \\
67 \\
0^{--} \\
261 \\
70 \\
115 \\
7^{--} \\
7_{515}^{-}\end{array}$ & 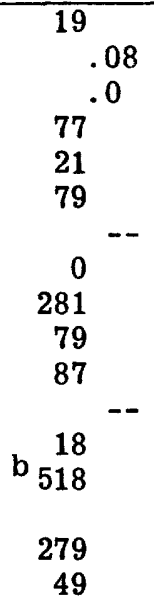 & \begin{tabular}{l}
44 \\
.15 \\
.10 \\
73 \\
25 \\
104 \\
\multicolumn{2}{c}{0} \\
386 \\
74 \\
108 \\
b 0 \\
618 \\
.0 \\
285 \\
0
\end{tabular} \\
\hline 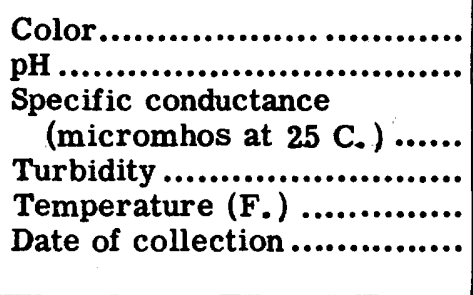 & $\begin{array}{r}0 \\
7.7 \\
\\
-- \\
0 \\
-- \\
\text { Oct. } 26, \\
1949\end{array}$ & $\begin{array}{r}0 \\
7.3 \\
\\
-- \\
0 \\
-- \\
\text { Oct. } 26, \\
1949\end{array}$ & $\begin{array}{r}0 \\
7.5 \\
\\
-- \\
0 \\
-- \\
\text { Oct. } 2 \hat{3}, \\
1949\end{array}$ & $\begin{array}{r}0 \\
7.5 \\
-- \\
0 \\
-- \\
\text { July } 14, \\
1949\end{array}$ \\
\hline 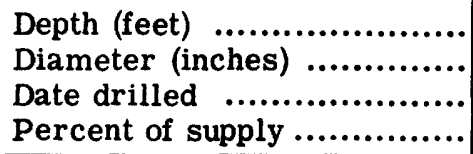 & $\begin{array}{l}-- \\
-- \\
-- \\
--\end{array}$ & $\begin{array}{r}403 \\
18 \\
1938 \\
--\end{array}$ & $\begin{array}{r}532 \\
18 \\
1945 \\
--\end{array}$ & $\begin{array}{r}495 \\
18 \\
1949 \\
--\end{array}$ \\
\hline
\end{tabular}

a Composite sample.

${ }^{b}$ Sum of determined constituents. 


\section{CALIFORNIA}

\section{LONG BEACH}

(Pcpulation, 250, 767)

Ownership: Municipal; supplies also about 20,000 people outside the city limits. Total population supplied, about 271,000 .

Source: 27 wells (Alamitos wells 8, 9, and 13; Citizens wells 5 to 7; Development wells 3 to 8 ; Wilson well; Wise wells 1 and 2; Commission wells 1 to 6 ; and North Long Beach wells 1, and 3 to 7), 58 percent of supply; Colorado River distributed by the Metropolitan Water District of Southern California, 42 percent of supply. (See Los Angeles.) The depth of the wells is reported to range from 193 to $1,700 \mathrm{ft}$, and the yield, from 190 to $1,925 \mathrm{gpm}$.

Treatment: Well water: prechlorination, coagulation with ferric chloride, diatomaceous earth, activated carbon, caustic soda, Calgon, sedimentation, rapid sand filtration, and postchlorination. Water from the Colorado River is softened and filtered by the Metropolitan Water District of Southern California. Rated capacity of treatment plant: 25, 000, 000 gipd.

Raw-water storage: $12,000,000$ gal.

Finished-water storage: $86,500,000$ gal.

\section{ANALYSES}

(Analyses, in parts per million, by City of Long Beach)

\begin{tabular}{|c|c|c|c|}
\hline & $\begin{array}{c}\text { Wells } \\
\text { (composite, } \\
\text { raw water) }\end{array}$ & $\begin{array}{c}\text { Wells } \\
\text { (composite, } \\
\text { finished water) }\end{array}$ & $\begin{array}{l}\text { Finished } \\
\text { water }^{\mathrm{a}}\end{array}$ \\
\hline Silica $\left(\mathrm{SiO}_{2}\right) \ldots . .$. & 11 & 22 & 18 \\
\hline Iron $(\mathrm{Fe})$ & .06 & .08 & .28 \\
\hline Manganese (Mn) .................... & .0 & .01 & .06 \\
\hline Calcium (Ca) ......................... & 13 & 12 & 18 \\
\hline Magnesium $(\mathrm{Mg}), \ldots \ldots \ldots \ldots \ldots \ldots \ldots$ & .9 & .9 & 4.4 \\
\hline Sodium (Na) ......................... & 71 & 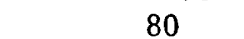 & 109 \\
\hline Potassium (K) .... & -1 & - & - \\
\hline 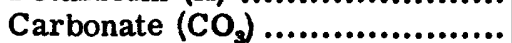 & 3 & 2 & 0 \\
\hline Bicarbonate $\left(\mathrm{HCO}_{3}\right) \ldots \ldots \ldots \ldots \ldots \ldots$ & 175 & 172 & 150 \\
\hline Sulfate $\left(\mathrm{SO}_{4}\right) \ldots \ldots \ldots \ldots \ldots \ldots \ldots \ldots \ldots$ & 8.4 & 8.7 & 88 \\
\hline Chloride (Cl) ......................... & 22 & 39 & 33 \\
\hline 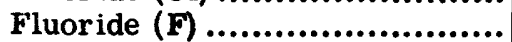 & .0 & .0 & .0 \\
\hline 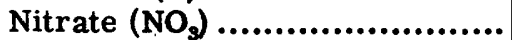 & & & \\
\hline Dissolved solids..........$\ldots \ldots \ldots$ & $\mathrm{D}_{216}$ & b 249 & ${ }^{\mathrm{D}} 375$ \\
\hline Hardness as $\mathrm{CaCO}_{3}$ : & & & \\
\hline Total ................... & 36 & 36 & 63 \\
\hline Noncarbonate ....................... & 0 & 0 & 0 \\
\hline Color & 80 & 8 & 17 \\
\hline pH... & 8.4 & 8.4 & 8.1 \\
\hline Specific conductance & & & \\
\hline (micromhos at 25 C. ).... & $\overline{--}$ & -- & -- \\
\hline Turbidity.................... & 77 & 7.9 & -- \\
\hline Temperature (F.) & & -- & $=-$ \\
\hline Date of collection ................... & Oct. 2, 1951 & Oct. 2, 1951 & Sept. 4, 1951 \\
\hline
\end{tabular}

b Composite, wells and Colorado River, from distribution system.

Sum of determined constituents. 


\section{CALIFORNIA}

\section{LOS ANGELES}

(Population, 1, 970, 358)

Ownership: Municipal; supplies also about 25,000 people outside the city limits. Total population supplied, about 2,000,000.

Source: Owens Valley Aqueduct (76 percent of supply); Los Angeles River sources (10 percent of supply); Vanowen wells (8 percent of supply); miscellaneous local wells ( 5 percent of supply); Colorado River distributed by Metropolitan Water District of Southern California (1 percent of supply); emergency supply from about 20 wells.

Treatment: Chlorination (except for a part of the supply from the emergency wells, which is not treated). All major reservoirs are chlorinated at the outlets; occasional chlorine residuals are carried from one reservoir to another.

Raw-water storage: Crowley Lake, 60, 000,000,000 gal; Haiwee Reservoir, 19, 500, 000, 000 gal; Bouquet Canyon Reservoir, 11, 750, 000, 000 gal; Lower San Fernando Reservoir, 6, 540,000,000 gal; Chatsworth Reservoir, $3,260,000,000$ gal; other smaller reservoirs.

Finished-water storage: Many minor reservoirs and tanks for pressure regulation. Total storage of both raw and treated water, 131, 000, 000, 000 gal.

The Owens Valley Aqueduct sources include 5 streams in Mono Basin, Owens River, and at times about 100 deep wells in Owens Valley. The Mono Basin streams are diverted through an 11 mile tunnel into Owens River, which is impounded in Crowley Lake, with a capacity of $60,000,000,000$ gal. These sources are combined in the aqueduct to supply the San Fernando Valley, the southwest part of Los Angeles proper, and is mixed with other sources in the remaining parts of the city. The total length of the Owens Valley aqueduct (including the tunnel) is 244 miles.

The Los Angeles River sources include spreading grounds, infiltration gallery, and 3 groups of wells: Verdugo, Headworiss, and Crystal Springs wells. 


\section{CALIFORNIA}

\section{ANALYSES}

(Analyses, in parts per million, by City of Los Angeles)

\begin{tabular}{|c|c|c|c|}
\hline & $\begin{array}{l}\text { Owens Valley } \\
\text { Aqueduct } \mathrm{a}\end{array}$ & $\begin{array}{c}\text { Los Angeles } \\
\text { River Conduit }\end{array}$ & $\begin{array}{c}\text { Vanowen } \\
\text { Wells }\end{array}$ \\
\hline 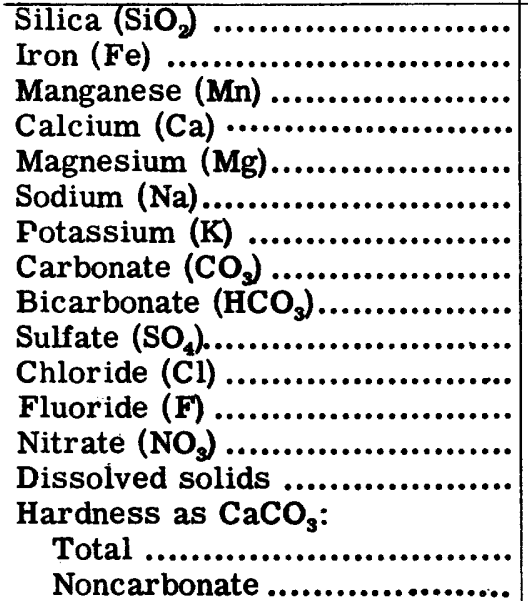 & 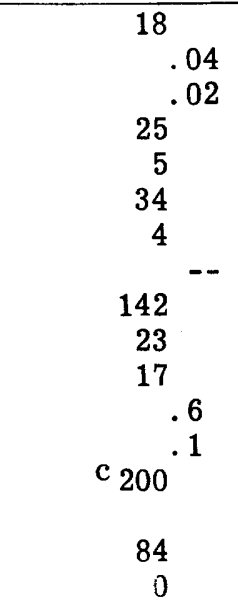 & \begin{tabular}{l}
23 \\
\multicolumn{2}{c}{.02} \\
$77^{.00}$ \\
20 \\
52 \\
4 \\
${ }^{2}$ \\
231 \\
139 \\
36 \\
${ }^{--}$ \\
12 \\
478 \\
\\
274 \\
84
\end{tabular} & $\begin{array}{c}24 \\
.00 \\
58 \\
-- \\
16 \\
33 \\
3 \\
\\
192 \\
96 \\
18 \\
.- \\
4.9 \\
350 \\
\\
210 \\
53\end{array}$ \\
\hline 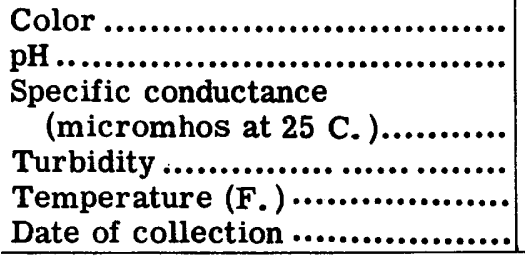 & $\begin{array}{r}-- \\
8.3 \\
320 \\
-- \\
58 \\
--\end{array}$ & $\begin{array}{r}-- \\
7.6 \\
720 \\
-- \\
65 \\
-- \\
\end{array}$ & $\begin{array}{r}-- \\
7.7 \\
540 \\
-- \\
63 \\
-- \\
\end{array}$ \\
\hline 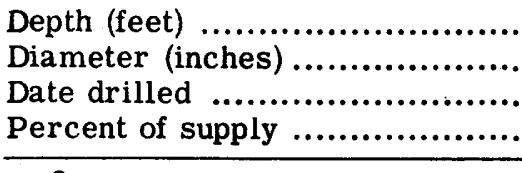 & &.. & $\begin{array}{r}257-395 \\
-- \\
-- \\
8\end{array}$ \\
\hline
\end{tabular}

average for 1950-51 fiscal year.

$b_{\text {This analysis was calculated according to the percentage derived from }}$ each of the sources. During the year the conduit may be supplied from Los Angeles River sources, Owens Valley Aqueduct, and Vanowen wells.

${ }^{\mathrm{c}}$ Sum of determined constituents. 


\title{
CALIFORNIA
}

\author{
LOS ANGELES--Continued
}

\section{ME TROPOLITAN WATER DISTRICT OF SOUTHERN CALIFORNIA}

Ownership: Metropolitan Water District of Southern California; supplies 16 constituent areas in 5 counties (Los Angeles, Orange, Riverside, San Bernardino, and San Diego) in amounts from 0 to 98 percent of supply; 22 percent of the total water production for these areas. Total population supplied, about 3, 563, 000 (estimated as of July 1949).

Source: Colorado River impounded in Lake Havasu. Emergency supply from San Gabriel River impounded in Morris Reservoir.

Treatment: La Verne plant (all Colorado River water except that through San Diego Aqueduct): treatment varies somewhat, but in general is as follows: prechlorination, activated carbon, coagulation with chlorine-activated silica sol (sodium silicate), intermittent partial lime softening, rapid sand filtration, zeolite or polystyrene resin softening of part of the water so that the total effluent has a hardness of about $125 \mathrm{ppm}$, postchlorination, final adjustment of $\mathrm{pH}$ with lime. San Diego Aqueduct: chlorination.

Rated capacity of treatment plant: $200,000,000 \mathrm{gpd}$ (designed to be increased to $400,000,000 \mathrm{gpd})$.

Raw-water storage: Lake Havasu, 233, 600, 000, 000 gal; Copper Basin Reservoir, 7, 886, 000, 000 gal; Lake Mathews, 34, 870,000, 000 gal; Morris Reservoir, 12, 810, 000, 000 gal; Gene Reservoir, 2, 050, 000, 000 gal; San Jacinto Reservoir, 554, 000, 000 gal.

Finished-water storage: Palos Verdes Reservoir, 326, 000, 000 gal; Orange County Reservoir, 65, 000, 000 gal; Corona Del Mar Reservoir, 4, 900, 000 gal.

Constituent areas of Metropolitan Water District of Southern California: Anaheim, Beverly Hills, Burbank, Coastal Municipal Water District, Compton, Fullerton, Glendale, Long Beach, Los Angeles, Pasadena, San Diego County Water Authority, San Marino, Santa Ana, Santa Monica, Torrance, and West Basin Municipal Water District (new area in M. W. D. June 1949). The West Basin Municipal Water District comprises the cities of El Segundo, Gardena, Hermosa Beach, Manhattan Beach, Redondo Beach, Palos Verdes Estates, and industrially important unincorporated areas.

Water is delivered from Lake Havasu by main aqueduct to San Diego Aqueduct and to Lake Mathews. San Diego Aqueduct connects with the main aqueduct and extends to San Vicente Reservoir of the San Diego system, a distance of 71.1 miles. The main aqueduct is 242 miles long; the total distribution system is 215 miles long, making a total aqueduct length of 457 miles. 


\section{CALIFORNIA}

\section{ANALYSES}

(Analyses, in parts per millions, by Metropolitan Water District of Southern Calif.)

\begin{tabular}{|c|c|c|c|c|c|}
\hline & $\begin{array}{l}\text { Raw } \\
\text { water a }\end{array}$ & $\begin{array}{r}\text { Finished } \\
\text { water a }\end{array}$ & & $\begin{array}{c}\text { Raw } \\
\text { water a }\end{array}$ & $\begin{array}{r}\text { Finished } \\
\text { water a }\end{array}$ \\
\hline $\begin{array}{l}\text { Silica }\left(\mathrm{SiO}_{2}\right) \ldots \ldots \ldots \ldots \\
\text { Iron }(\mathrm{Fe}) \quad \ldots \ldots \ldots \ldots . . . \\
\text { Manganese }(\mathrm{Mn}) \ldots \ldots\end{array}$ & $\begin{array}{r}8.0 \\
--\end{array}$ & 12 & $\begin{array}{r}\text { Hardness as } \mathrm{CaCO}_{3} \text { : } \\
\text { Total .................. } \\
\text { Noncarbonate...... }\end{array}$ & $\begin{array}{l}315 \\
197\end{array}$ & $\begin{array}{r}125 \\
6 \\
\end{array}$ \\
\hline $\begin{array}{l}\text { Calcium (Ca) ......... } \\
\text { Magnesium }(\mathrm{Mg}) . . . .\end{array}$ & $\begin{array}{l}79 \\
28\end{array}$ & $\begin{array}{l}31 \\
12\end{array}$ & Color.................... & -- & - \\
\hline $\begin{array}{l}\text { Sodium (Na) .......... } \\
\text { Potassium (K) } \ldots \ldots \ldots\end{array}$ & $\begin{array}{r}99 \\
4\end{array}$ & $\begin{array}{r}189 \\
3\end{array}$ & 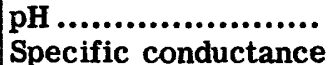 & 8.4 & 8.8 \\
\hline Carbonate $\left(\mathrm{CO}_{3}\right) \ldots$ & 4 & 12 & (micromhos at & & \\
\hline Bicarbonate $\left(\mathrm{HCO}_{3}\right)$ & 137 & 121 & 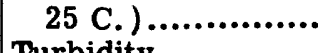 & 1,040 & 1,100 \\
\hline $\begin{array}{l}\text { Sulfate }\left(\mathrm{SO}_{4}\right) \ldots \ldots \ldots \ldots \\
\text { Chloride }(\mathrm{Cl}) \ldots \ldots \ldots\end{array}$ & 290 & 290 & Turbidity ................ & -- & \\
\hline $\begin{array}{l}\text { Chloride }(C 1) \ldots \ldots \ldots \\
\text { Fluoride }(F) \ldots \ldots . . .\end{array}$ & 79 & 83 & $\begin{array}{l}\text { Temperature (F.)... } \\
\text { Date of collection... }\end{array}$ & -- & -- \\
\hline $\begin{array}{l}\text { Nitrate }\left(\mathrm{NO}_{3}\right) \text {.......... } \\
\text { Dissolved solids..... }\end{array}$ & $\mathrm{b}_{661} \cdot 2$ & $b_{692}^{.2}$ & & & \\
\hline
\end{tabular}

Regular determinations at treatment plant,1948-49

\begin{tabular}{|c|c|c|c|c|c|c|c|c|c|c|c|c|}
\hline & \multicolumn{3}{|c|}{$\begin{array}{l}\text { Alkalinity } \\
\text { as } \mathrm{CaCO}_{3} \\
\text { (ppm) }\end{array}$} & \multicolumn{3}{|c|}{ pH } & \multicolumn{3}{|c|}{$\begin{array}{c}\text { Hardness } \\
\text { as CaCO } \\
\text { (ppm) }\end{array}$} & \multicolumn{3}{|c|}{ Turbidity } \\
\hline & $A \nabla$ & $\operatorname{Max}$ & Min & Av & $\operatorname{Max}$ & Min & Av & Max & Min & Av & $\operatorname{Max}$ & Min \\
\hline $\begin{array}{l}\text { Raw water.......... } \\
\text { Finished water... }\end{array}$ & $\begin{array}{l}119 \\
111\end{array}$ & $\begin{array}{l}127 \\
151\end{array}$ & $\begin{array}{r}108 \\
63\end{array}$ & $\begin{array}{l}8.3 \\
8.8\end{array}$ & $\begin{array}{l}8.6 \\
9.3\end{array}$ & $\begin{array}{l}8.0 \\
8.4\end{array}$ & $\begin{array}{l}334 \\
133\end{array}$ & $\begin{array}{l}343 \\
196\end{array}$ & $\begin{array}{l}319 \\
116\end{array}$ & $\begin{array}{l}-- \\
--\end{array}$ & $\begin{array}{l}-- \\
--\end{array}$ & -- \\
\hline
\end{tabular}

${ }^{a}$ Colorado River. Average for 1950-51 fiscal year.

${ }^{b}$ Sum of determined constituents. 


\section{CALIFORNIA}

\section{LYNWOOD}

(Population, 25, 823)

Ownership: Municipal; supplies also about 500 people outside the city limits.

Total population supplied, about 26,300 .

Source: 11 wells ( 2 to 12 ) ranging in depth from 352 to $956 \mathrm{ft}$. The yield of the wells is reported to range from 550 to $1,700 \mathrm{gpm}$, and to average 1,158 .

Treatment: None.

Storage: Elevated tanks, 300, 000 gal.

\section{ANALYSES}

(Analyses, in parts per million, by California State Dept. of Public Health)

\begin{tabular}{|c|c|c|c|c|c|}
\hline & Well 2 & Well 3 & Well 4 & Well 7 & Well 8 \\
\hline 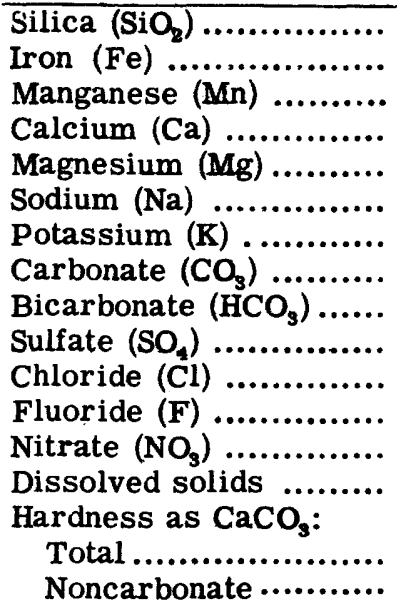 & 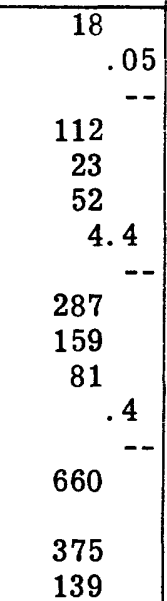 & $\begin{array}{c}22 \\
-- \\
77 \\
16 \\
49 \\
4.1 \\
-- \\
268 \\
99 \\
36 \\
.3 \\
448^{--}\end{array}$ & 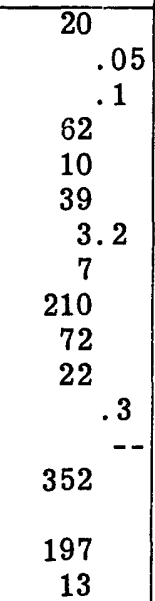 & $\begin{array}{c}23 \\
-- \\
58 \\
12 \\
12 \\
31 \\
2.4 \\
2 \\
248 \\
37 \\
17 \\
.3 \\
1.1 \\
310\end{array}$ & $\begin{array}{c}20 \\
-- \\
54 \\
8.6 \\
37 \\
4.0 \\
2 \\
237 \\
42 \\
15 \\
.3 \\
.3 \\
306 \\
171 \\
0\end{array}$ \\
\hline 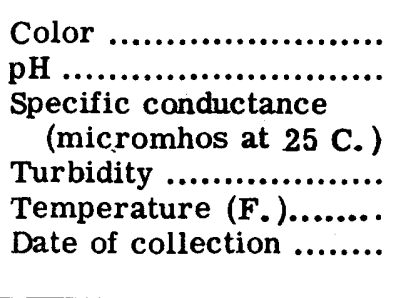 & $\begin{array}{r}0 \\
8.2 \\
910 \\
.6 \\
-- \\
\text { Apr. } 27, \\
1951\end{array}$ & $\begin{array}{r}0 \\
8.3 \\
\\
656 \\
.5 \\
-- \\
\text { Apr. } 27, \\
1951\end{array}$ & $\begin{array}{r}0 \\
8.9 \\
517 \\
.5 \\
-- \\
\text { Apr. } 27, \\
1951\end{array}$ & $\begin{array}{r}0 \\
8.5 \\
471 \\
.05 \\
-- \\
\text { Apr. } 27, \\
1951\end{array}$ & $\begin{array}{r}0 \\
8.6 \\
454 \\
\\
.3 \\
-- \\
\text { Apr. } 27, \\
1951\end{array}$ \\
\hline $\begin{array}{l}\text { Depth (feet) } \\
\text { Diameter (inches)........... } \\
\text { Date drilled ............... } \\
\text { Percent of supply ........ }\end{array}$ & $\begin{array}{r}352 \\
16 \\
1930 \\
--\end{array}$ & $\begin{array}{r}436 \\
16 \\
1924 \\
--\end{array}$ & $\begin{array}{r}790 \\
16 \\
1946 \\
--\end{array}$ & $\begin{array}{r}610 \\
16 \\
1907 \\
--\end{array}$ & $\begin{array}{r}864 \\
16 \\
1948 \\
--\end{array}$ \\
\hline
\end{tabular}




\section{CALIFORNIA}

\section{MANHATTAN BEACH}

(Population, 17, 330)

Ownership: Municipal; supplies also about 7,200 people outside the city limits. Total population supplied, about 24,500 .

Source: 6 wells ( 9 to 14 ), 70 percent of supply; Colorado River distributed by the Metropolitan Water District of Southern California, 30 percent of supply. (See Los Angeles.) Well 9 is reported to be $390 \mathrm{ft}$ deep, and wells 10 to 14 are reported to be $550 \mathrm{ft}$ deep. The yield of each well is reported to be $800 \mathrm{gpm}$.

Treatment: The well water is not treated. The Colorado River water is softened and filtered by the Metropolitan Water District of Southern California.

Raw-water storage: None.

Finished-water storage: 2,850,000 gal.

\section{ANALYSES}

(Analyses, in parts per million, by Carl Wilson, Los Angeles)

\begin{tabular}{|c|c|c|c|c|c|}
\hline & Well 9 & Well 10 & Well 11 & Well 12 & Well 13 \\
\hline 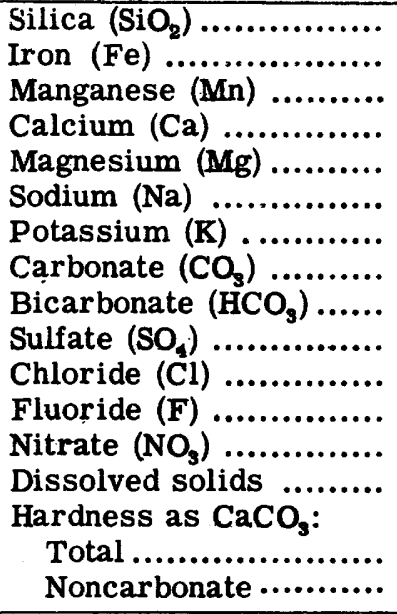 & $\begin{array}{r}19 \\
-- \\
0 \\
65 \\
18 \\
76 \\
-- \\
0 \\
287 \\
28 \\
98 \\
-- \\
0 \\
445 \\
236 \\
0\end{array}$ & \begin{tabular}{rr|}
25 & .02 \\
52 & -- \\
17 & \\
94 & \\
& -- \\
0 & \\
417 & \\
3 & \\
43 & \\
& -- \\
0 & \\
439 & \\
200 & \\
0 & \\
\end{tabular} & $\begin{array}{r}25 \\
-- \\
0 \\
42 \\
17 \\
86 \\
-- \\
0 \\
378 \\
1 \\
37 \\
-- \\
-- \\
\text { a } 394\end{array}$ & 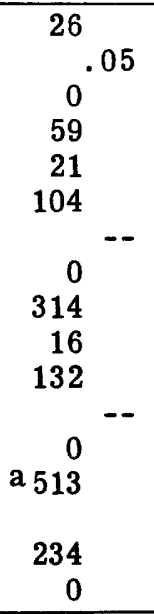 & \begin{tabular}{c}
22 \\
\multicolumn{2}{c}{.02} \\
0 \\
67 \\
17 \\
80 \\
\multicolumn{2}{c}{--} \\
0 \\
298 \\
35 \\
93 \\
\\
0 \\
0 \\
461 \\
\\
237 \\
0 \\
\end{tabular} \\
\hline 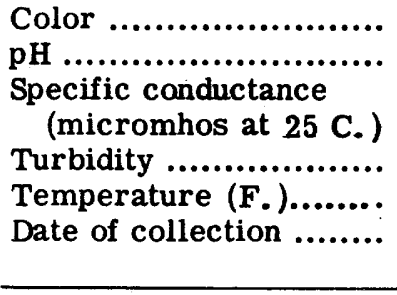 & $\begin{array}{r}7.8 \\
-- \\
-- \\
-- \\
\text { June } 4, \\
1951 \\
\end{array}$ & $\begin{array}{r}7.8 \\
-- \\
-- \\
-- \\
\text { June } 4, \\
1951 \\
\end{array}$ & $\begin{array}{r}7.7 \\
-- \\
-- \\
-- \\
\text { June } 4, \\
1951\end{array}$ & $\begin{array}{r}7.5 \\
-- \\
-- \\
-- \\
\text { June } 4, \\
1951\end{array}$ & $\begin{array}{r}7.7 \\
-- \\
-- \\
-- \\
\text { June } 4, \\
1951\end{array}$ \\
\hline $\begin{array}{l}\text { Depth (feet) } \ldots . . . \ldots \ldots \ldots . . . \\
\text { Diameter (inches)........ } \\
\text { Date drilled .............. } \\
\text { Percent of supply ........ }\end{array}$ & $\begin{array}{r}390 \\
16 \\
1940 \\
-- \\
\end{array}$ & $\begin{array}{r}550 \\
16 \\
1943 \\
-- \\
\end{array}$ & $\begin{array}{r}550 \\
16 \\
1943 \\
--\end{array}$ & $\begin{array}{r}550 \\
16 \\
1948 \\
-- \\
\end{array}$ & $\begin{array}{r}550 \\
16 \\
1949 \\
-- \\
\end{array}$ \\
\hline
\end{tabular}

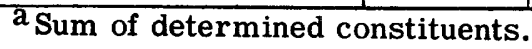




\section{CALIFORNIA}

MONROVIA

(Population, 20, 186)

Ownership: Municipal.

Source: 6 wells (San Gabriel 1 to 4 , Chapman 5 and 6), 476, 420, 500, 530, 387, and $424 \mathrm{ft}$ deep, 94 percent of supply; 2 springs, 6 percent of supply. Auxiliary supply, surface water runoff from mountains when flow is adequate.

Treatment: Mountain and spring supplies are chlorinated. Well water is not treated.

Storage: $8,000,000$ gal.

\section{ANALYSES}

(Analyses, in parts per million, by California State Dept. of Public Health)

\begin{tabular}{|c|c|c|c|}
\hline & $\begin{array}{c}\text { San Gabriel } \\
\text { Well } 2\end{array}$ & $\begin{array}{c}\text { Chapman } \\
\text { Well } 6\end{array}$ & $\begin{array}{c}\text { Surface water } \\
\text { run-off }\end{array}$ \\
\hline 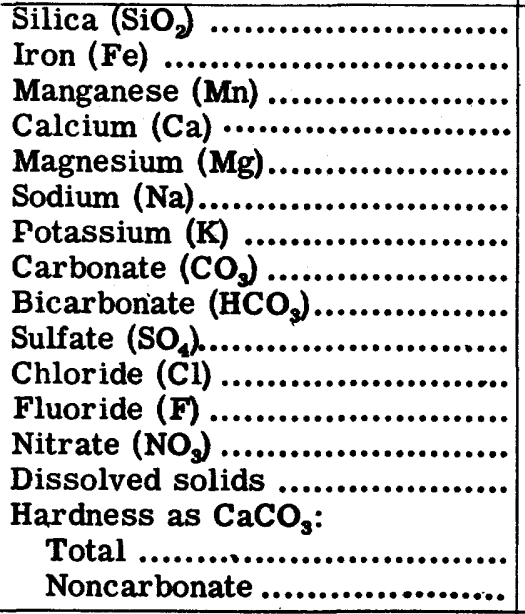 & $\begin{array}{c}18 \\
49 \\
15 \\
14 \\
1.0 \\
-- \\
193 \\
14 \\
12 \\
16 \\
a^{--} \\
235 \\
185 \\
26 \\
\end{array}$ & $\begin{array}{c}20 \\
.01 \\
42 \\
11 \\
20 \\
3.5 \\
-- \\
204 \\
10 \\
9.5 \\
1.0 \\
8.0 \\
228 \\
151 \\
0 \\
\end{array}$ & $\begin{array}{c}18 \\
52 \\
-- \\
15 \\
17 \\
3.0 \\
-- \\
244^{--} \\
20 \\
8.5 \\
.4 \\
.4 \\
270^{.5} \\
190 \\
0\end{array}$ \\
\hline 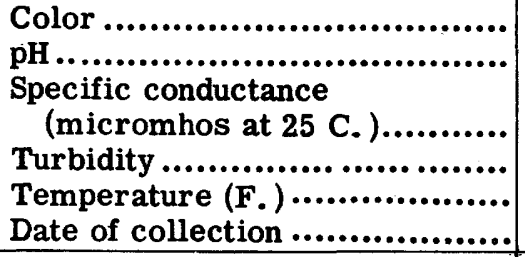 & $\begin{array}{r}7.5 \\
395 \\
1.3 \\
-- \\
\text { May 4, } 1951 \\
\end{array}$ & $\begin{array}{r}7.7 \\
362 \\
1.2 \\
--- \\
\text { May } 4,1951 \\
\end{array}$ & $\begin{array}{r}8 . \overline{2} \\
408 \\
.9 \\
-- \\
\text { May } 4,1951 \\
\end{array}$ \\
\hline 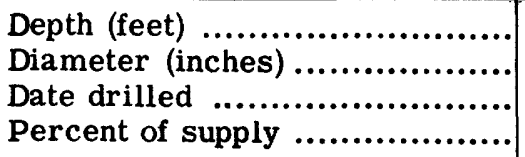 & $\begin{array}{r}420 \\
20 \\
1924 \\
--\end{array}$ & $\begin{array}{r}424 \\
20 \\
1928 \\
--\end{array}$ & $\begin{array}{l}-- \\
-- \\
--\end{array}$ \\
\hline
\end{tabular}




\section{CALIFORNIA}

\section{MONTEREY}

(Population, 16, 205)

Ownership: California Water and Telephone Co.; supplies also Pacific Grove, Carmel, and about 30,000 people in other communities. Total population supplied, about 60,000 .

Source: Carmel River (99 percent of supply) and 2 wells, 80 and $60 \mathrm{ft}$ deep (1 percent of supply).

Treatment: Alum, lime, and activated carbon as needed, pressure filtration, and chlorination.

Rated capacity of treatment plant: 8,000,000 gpd.

Raw-water storage: $1,532,000,000$ gal.

Finished-water storage: 170,000,000 gal.

\section{ANALYSES}

(Analyses, in parts per million, by California State Dept. of Public Health)

\begin{tabular}{|c|c|c|c|c|c|}
\hline & $\begin{array}{c}\text { Raw } \\
\text { water a }\end{array}$ & $\begin{array}{c}\text { Finished } \\
\text { water a }\end{array}$ & & $\begin{array}{c}\text { Raw } \\
\text { water a }\end{array}$ & $\begin{array}{l}\text { Finished } \\
\text { water a }\end{array}$ \\
\hline 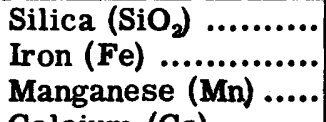 & \multirow{7}{*}{\begin{tabular}{c}
$0 . \overline{10}$ \\
.0 \\
16 \\
8.9 \\
13 \\
\multicolumn{2}{c}{${ }^{--}$} \\
73 \\
18 \\
9.5 \\
.3 \\
$154^{.0}$
\end{tabular}} & \multirow{7}{*}{$\begin{array}{c}0.04 \\
.0 \\
13 \\
.0 \\
10 \\
12 \\
\\
{ }^{2} \\
0 \\
73 \\
15 \\
9.0 \\
.2 \\
.0\end{array}$} & $\begin{array}{r}\text { Hardness as } \mathrm{CaCO}_{3}: \\
\text { Total .................. } \\
\text { Noncarbonate...... }\end{array}$ & $\begin{array}{l}77 \\
17 \\
\end{array}$ & $\begin{array}{l}76 \\
14 \\
\end{array}$ \\
\hline $\begin{array}{l}\text { Calcium }(\mathrm{Ca}) \text {.......... } \\
\text { Magnesium }(\mathrm{Mg}) . . . .\end{array}$ & & & Color....................... & -- & -- \\
\hline $\begin{array}{l}\text { Sodium }(\mathrm{Na}) \\
\text { Potassium }(\mathrm{K}) \\
\text { Carbonate }(\mathrm{CO}, \ldots \ldots\end{array}$ & & & $\begin{array}{l}\text { pHecific conductance } \\
\text { (micromhos at }\end{array}$ & 7.8 & 7.8 \\
\hline Bicarbonate $\left(\mathrm{HCO}_{3}\right)$ & & & 25 C. $) \ldots \ldots \ldots \ldots$ & -- & -- \\
\hline $\begin{array}{l}\text { Sulfate }\left(\mathrm{SO}_{4}\right) \ldots \ldots \ldots \\
\text { Chloride }(\mathrm{Cl}) \ldots \ldots . . . .\end{array}$ & & & $\begin{array}{l}\text { Turbidity ............... } \\
\text { Temperature (F.)... }\end{array}$ & -- & -- \\
\hline $\begin{array}{l}\text { Fluoride }(\mathrm{F}) \\
\text { Nitrate }\left(\mathrm{NO}_{3}\right) \ldots \ldots \ldots \ldots\end{array}$ & & & Date of collection... & $\begin{array}{c}\text { May } 8 \\
1951\end{array}$ & $\begin{array}{l}\text { May 8, } \\
1951\end{array}$ \\
\hline Dissolved so & & & & & \\
\hline
\end{tabular}

${ }^{\text {a Carmel River. }}$ 


\section{CALIFORNIA}

MONTEREY PARK

(Population, 20, 395)

Ownership: Municipal.

Source: 4 wells (1 to 4 ), 410, 450,1,110, and $480 \mathrm{ft}$ deep. The yield of the wells is reported to be $900,1,300,2,000$, and $900 \mathrm{gpm}$. Emergency supply from Southern California Water Co.

Treatment: None.

Storage: Reservoirs, 4, 000, 000 gal.

\section{ANALYSES}

(Analyses, in parts per million, by California State Dept. of Public Health)

\begin{tabular}{|c|c|c|c|c|}
\hline & Well 1 & Well 2 & Well 3 & Well 4 \\
\hline 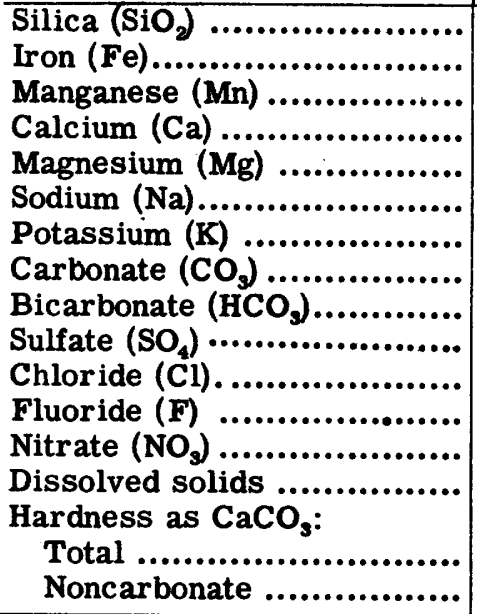 & $\begin{array}{c}22 \\
--- \\
35 \\
11 \\
28 \\
2.0 \\
-- \\
a_{173} \\
22 \\
21 \\
.6 \\
7.5 \\
272 \\
133 \\
0\end{array}$ & $\begin{array}{c}19 \\
.02 \\
39 \\
-- \\
10 \\
28 \\
2.0 \\
a^{-} \\
176 \\
14 \\
20 \\
.6 \\
12 \\
262 \\
140 \\
0\end{array}$ & $\begin{array}{c}20 \\
30 \\
5.3 \\
45 \\
2.0 \\
-- \\
a_{160}^{--} \\
22 \\
23 \\
.5 \\
4.9 \\
280 \\
96 \\
0\end{array}$ & $\begin{array}{c}12 \\
.03 \\
38 \\
12 \\
30 \\
2.6 \\
a_{185}^{--} \\
14 \\
24 \\
14 \\
284 \\
143 \\
0\end{array}$ \\
\hline 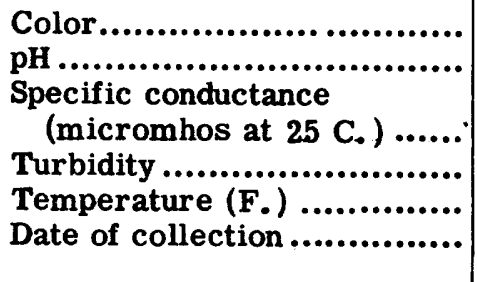 & $\begin{array}{r}0 \\
8.2 \\
361 \\
1.0 \\
\text { Apr. } 1 \overline{8} \\
1951\end{array}$ & $\begin{array}{r}0 \\
8.4 \\
367 \\
.8 \\
\text { Apr. } 1 \overline{8} \\
1951\end{array}$ & $\begin{array}{r}0 \\
8.6 \\
364 \\
.8 \\
\text { Apr. } 1 \overline{8} \\
1951\end{array}$ & $\begin{array}{r}0 \\
8.4 \\
374 \\
.9 \\
\text { Apr. } 1 \overline{8}^{-} \\
1951\end{array}$ \\
\hline 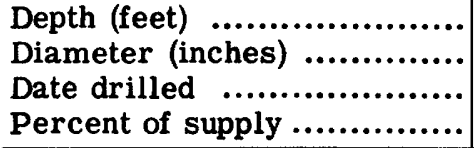 & $\begin{array}{r}410 \\
12 \\
1875 \\
--\end{array}$ & $\begin{array}{r}450 \\
16 \\
1924 \\
--\end{array}$ & $\begin{array}{r}1,110 \\
30-16 \\
1946 \\
--\end{array}$ & $\begin{array}{r}480 \\
12 \\
1945 \\
--\end{array}$ \\
\hline
\end{tabular}

\footnotetext{
${ }^{\mathrm{a}}$ Includes the equivalent of any carbonate $\left(\mathrm{CO}_{3}\right)$ present.
} 


\section{CALIFORNIA}

\section{NATIONAL CITY}

(Population, 21, 199)

Ownership: California Water and Telephone Co. ; supplies Chula Vista and also about 11,000 people outside the city limits. Total population supplied, about 48,000 .

Source: Colorado River distributed by the Metropolitan Water District of Southern California (92 percent of supply). (See Los Angeles.) National City well 1 (8 percent of supply). The well is $74 \mathrm{ft}$ deep and is reported to yield $450 \mathrm{gpm}$. Emergency supply from shallow wells in bed of Sweetwater River.

Treatment: Chlorination.

Raw-water storage: Sweetwater Reservoir, 9, 500, 000, 000 gal.

Finished-water storage: 790,000 gal.

\section{ANALYSIS}

(Analysis, in parts per million, by California State Dept. of Public Health)

\begin{tabular}{|c|c|c|c|}
\hline & Well 1 & & Well 1 \\
\hline \multirow{2}{*}{ 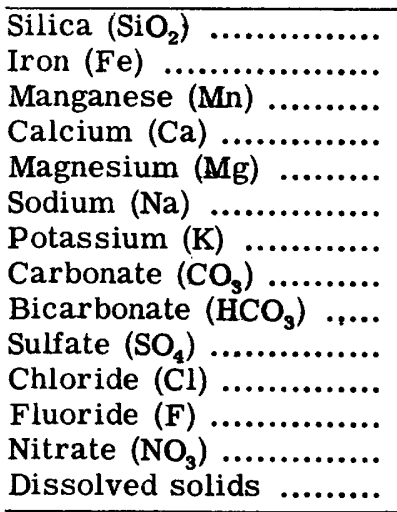 } & $\begin{array}{c}7.2 \\
.04 \\
\end{array}$ & $\begin{array}{r}\text { Hardness as } \mathrm{CaCO}_{3}: \\
\text { Total ...................... } \\
\text { Noncarbonate ........... } \\
\end{array}$ & $\begin{array}{r}180 \\
6 \\
\end{array}$ \\
\hline & $\begin{array}{r}40 \\
20 \\
132 \\
13 \\
0 \\
214 \\
61 \\
172 \\
.3 \\
a_{551} \\
\quad-- \\
\end{array}$ & 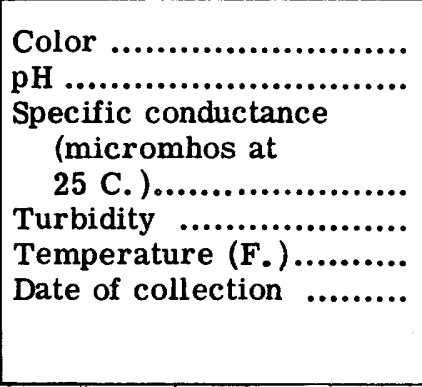 & $\begin{array}{c}\text { Feb. } 8 \text {, } \\
1951\end{array}$ \\
\hline 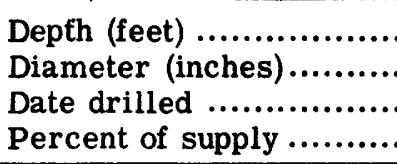 & 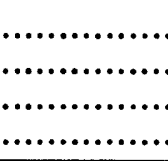 & 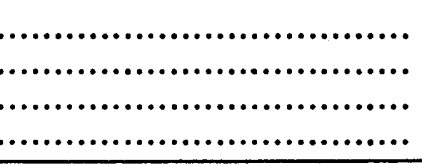 & $\begin{array}{r}74 \\
12 \\
1931 \\
8 \\
\end{array}$ \\
\hline
\end{tabular}

${ }^{\mathrm{a}}$ Sum of determined constituents. 


\title{
CALIFORNIA
}

\author{
OAKLAND \\ (Population, 384, 575)
}

Ownership: East Bay Municipal Utility District; supplies also Alameda, Albany, Berkeley, El Cerrito, Richmond, San Leandro, Vallejo (part of supply), other smaller cities, and numerous unincorporated areas in Alameda and Contra Costa Counties. Total population supplied, about 900,000 .

Source: Mokelumne River impounded in Pardee Reservoir (93 percent of supply); local runoff into San Pablo, Upper San Leandro, Chabot, and Lafayette Reservoirs (7 percent of supply).

Treatment: Orinda Plant (Mokelumne River): coagulation with alum, rapid sand filtration, chlorination, and lime for pH adjustment. San Pablo and Upper San Leandro Plants: aeration, coagulation with alum, sedimentation, rapid sand filtration, chlorination, and lime for $\mathrm{pH}$ adjustment. Chabot and Grant Miller (Lafayette Reservoir) Plants: coagulation with alum, sedimentation, pressure filtration, chlorination, lime for $\mathrm{pH}$ adjustment.

Rated capacity of treatment plants: $196,000,000 \mathrm{gpd}$.

Raw-water storage: Reservoirs: Pardee, San Pablo, Upper San Leandro, Chabot, and Lafayette, 102, 759, 000, 000 gal.

Finished-water storage: About 100 distribution reservoirs, 387, 900, 000 gal.

Pardee Reservoir, with a storage capacity of $68,400,000,000 \mathrm{gal}$, is about 94 miles northeast of the East Bay area. Water is released through an outlet tower into the twin Mokelumne Aqueducts, together capable of delivering almost $100,000,000$ gpd by gravity flow. By operating pumping plants the daily flow can be increased to more than 210,000,000 gpd. Most of this water is treated at the Orinda Filter Plant and transmitted into distribution mains; the remaining amounts are stored in the four terminal reservoirs.

The storage capacity of the terminal reservoirs is as follows: San Pablo, 14, 000, 000, $000 \mathrm{gal}$; Upper San Leandro, 13, 500, 000, 000 gal; Chabot, 4, 100, 000, 000 gal; Lafayette, $1,700,000,000$ gal.

Although much of the water is served by gravity, the district requires 67 pumping plants and over 100 distribution reservoirs to serve those at the higher elevations. 


\section{CALIFORNIA}

\section{ANALYSES}

(Analyses, in parts per million, by East Bay Municipal Utility District)

\begin{tabular}{|c|c|c|c|c|}
\hline & $\begin{array}{r}\text { Finished } \\
\text { water }\end{array}$ & $\begin{array}{c}\text { Finished } \\
\text { water }\end{array}$ & $\begin{array}{r}\text { Finished } \\
\text { water }\end{array}$ & $\begin{array}{c}\text { Finished } \\
\text { water } d\end{array}$ \\
\hline 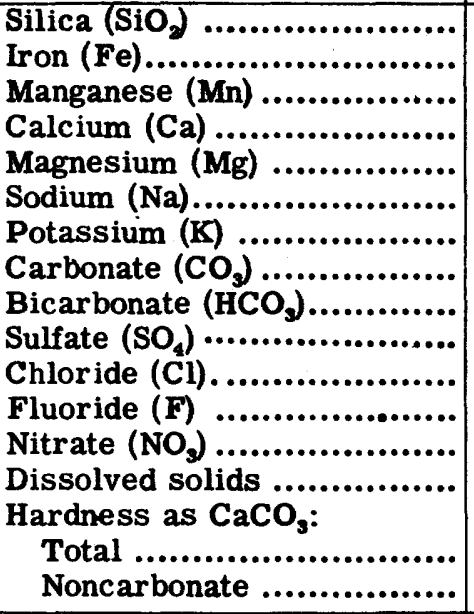 & $\begin{array}{r}7.2 \\
\mathrm{e} .4 \\
.0 \\
6.0 \\
.7 \\
4.7 \\
-- \\
\mathrm{f}^{2} \\
24 \\
1.1 \\
5.0 \\
.1 \\
\mathrm{~g}_{37}--\end{array}$ & $\begin{array}{l}e_{2.3}^{1.3} \\
.0 \\
23 \\
5.6 \\
13 \\
-- \\
81 \\
24 \\
13 \\
.1 \\
g_{122}^{--} \\
81 \\
15\end{array}$ & $\begin{array}{c}5.6 \\
e^{.6} \\
.0 \\
36 \\
13 \\
20 \\
-- \\
142 \\
45 \\
18 \\
.2 \\
-- \\
220^{--} \\
144 \\
.28\end{array}$ & $\begin{array}{l}e_{.9}^{.4} \\
.0 \\
48 \\
22 \\
34 \\
199 \\
71 \\
32 \\
.- \\
310^{--} \\
208 \\
45\end{array}$ \\
\hline 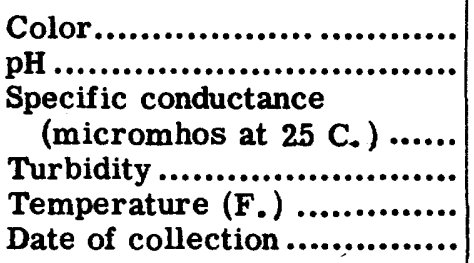 & $\begin{array}{r}9 . \overline{1} \\
-- \\
-- \\
-- \\
\text { June } 1950\end{array}$ & $\begin{array}{r}8.0 \\
-- \\
-- \\
\text { June } 1950\end{array}$ & $\begin{array}{r}7.9 \\
-- \\
-- \\
-- \\
\text { June } 1950\end{array}$ & June 1950 \\
\hline
\end{tabular}

${ }^{\text {a }}$ Mokelumne River.

${ }^{b}$ San Pablo Reservoir.

c Upper San Leandro Reservoir.

d Chabot Reservoir.

$\mathrm{e}$ Iron and aluminum oxides.

f Includes the equivalent of any carbonate $\left(\mathrm{CO}_{3}\right)$ as bicarbonate $\left(\mathrm{HCO}_{3}\right)$.

$\mathrm{g}_{\text {Sum of determined constituents. }}$ 


\title{
CALIFORNIA
}

\author{
ONTARIO \\ (Population, 22, 872)
}

Ownership: Municipal; supplies about 300 people outside the city limits. Total population supplied, about $23,200$.

Source: 8 wells ( 1 to 8 ), 600,600,604, 507, 551, 496, and $536 \mathrm{ft}$ deep. The yield of the wells is reported to be $945,1,650,1,500,1,600,2,400,2,400$, 1,420 and $450 \mathrm{gpm}$. Emergency supply from well and surface water of San Antonio Water Co.

Treatment: None.

Storage: $14,750,000$ gal.

\section{ANALYSES}

(Analyses, in parts per million, by California State Dept. of Public Health)

\begin{tabular}{|c|c|c|c|}
\hline & Well 1 & Well 6 & Well 7 \\
\hline 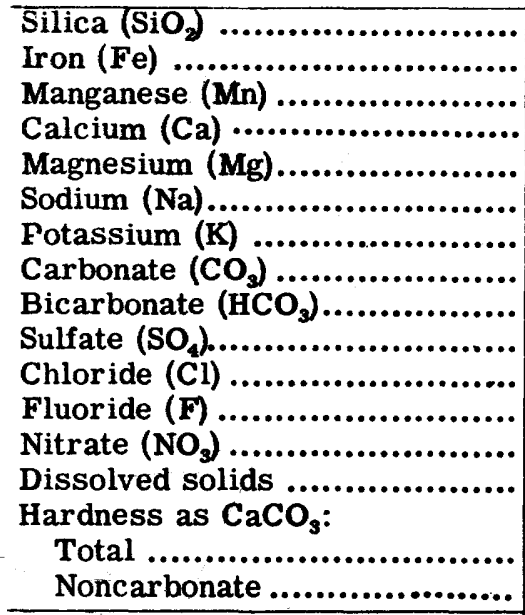 & $\begin{array}{c}27 \\
53 \\
53 \\
6.3 \\
15 \\
3.0 \\
0 \\
200 \\
9.5 \\
7.0 \\
.4 \\
1.9 \\
236 \\
158 \\
0 \\
\end{array}$ & $\begin{array}{c}29 \\
.02 \\
-- \\
47 \\
6.4 \\
16 \\
2.0 \\
0 \\
187 \\
8.0 \\
6.0 \\
.2 \\
2.5 \\
208 \\
144 \\
0 \\
\end{array}$ & $\begin{array}{c}34 \\
-- \\
46 \\
7.6 \\
18 \\
0^{--} \\
172 \\
14 \\
9 \\
.4 \\
2.6 \\
238 \\
145 \\
4 \\
\end{array}$ \\
\hline 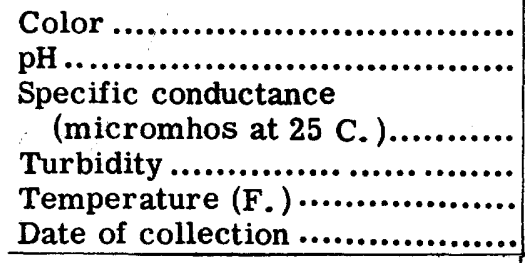 & $\begin{array}{r}0 \\
8.5 \\
\\
352 \\
1.6 \\
-- \\
\text { Apr. } 12,1951 \\
\end{array}$ & \begin{tabular}{r|}
0 \\
-- \\
327 \\
2.2 \\
-- \\
Apr. 13,1951 \\
\end{tabular} & $\begin{array}{r}0 \\
8.5 \\
\\
343 \\
1.7 \\
-- \\
\text { Apr. } 13,1951 \\
\end{array}$ \\
\hline 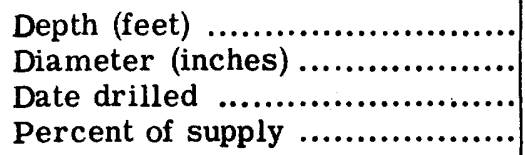 & $\begin{array}{r}600 \\
16 \\
1910 \\
--\end{array}$ & $\begin{array}{r}551 \\
26 \\
1930 \\
--\end{array}$ & $\begin{array}{r}496 \\
16 \\
-- \\
--\end{array}$ \\
\hline
\end{tabular}




\title{
CALIFORNIA
}

\author{
OXNARD \\ (Population, 21, 567)
}

Ownership: Municipal; supplies also about 2,000 people outside the city limits.

Total population supplied, about 23,600 .

Source: 5 wells ( 1 to 5), 234, 234, 232, $250 \mathrm{ft}$ deep (depth not reported for well 5).

The yield of the wells is reported to be $800,800,800,800$, and $1,000 \mathrm{gpm}$.

Treatment: None.

Storage: $1,000,000$ gal.

\section{ANALYSES}

(Analyses, in parts per million, by Smith-Emery Co., Los Angeles, Calif.)

\begin{tabular}{|c|c|c|c|}
\hline & $\begin{array}{c}\text { Wells } \\
\text { (compósite } \\
\text { sample) }\end{array}$ & Well 1 & Well 5 \\
\hline 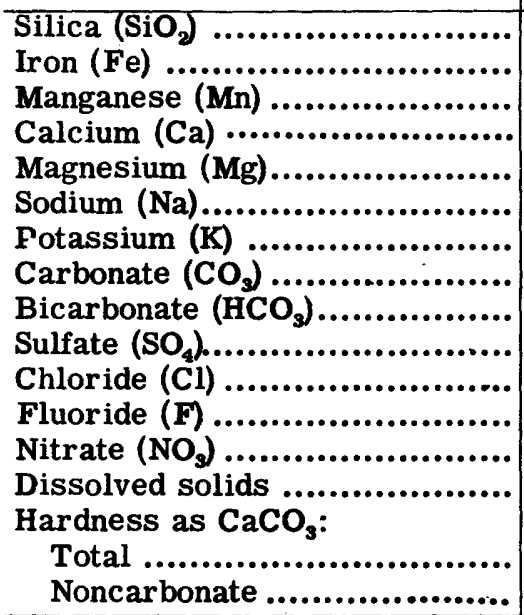 & $\begin{array}{c}7.5 \\
-- \\
155^{--} \\
42 \\
105 \\
3^{--} \\
262 \\
471 \\
55 \\
-- \\
a_{968}^{--} \\
561 \\
340\end{array}$ & $\begin{array}{l}.25 \\
.-- \\
153 \\
51 \\
86 \\
0^{--} \\
230 \\
501 \\
58 \\
.6 \\
6.0 \\
969 \\
594 \\
402\end{array}$ & $\begin{array}{c}0^{--} \\
107^{.8} \\
61 \\
93 \\
0^{--} \\
248 \\
625 \\
61 \\
.7 \\
2.4 \\
1,160 \\
745 \\
540\end{array}$ \\
\hline 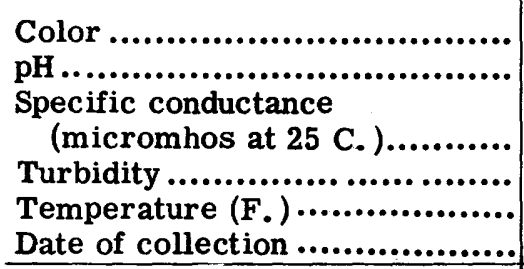 & $\begin{array}{r}8.2 \\
1,500 \\
-- \\
-- \\
\text { Nov. } 24,1950 \\
\end{array}$ & $\begin{array}{r}7.4 \\
-- \\
-- \\
-- \\
\text { Dec. } 8,1950 \\
\end{array}$ & $\begin{array}{r}-- \\
-- \\
-- \\
\text { Dec. } 8,1950 \\
\end{array}$ \\
\hline 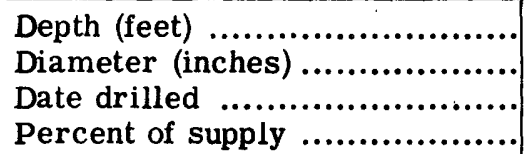 & $\begin{array}{l}-- \\
-- \\
--\end{array}$ & $\begin{array}{r}234 \\
14 \\
1912 \\
---\end{array}$ & $\begin{array}{l}-- \\
14 \\
-- \\
--\end{array}$ \\
\hline
\end{tabular}

${ }^{\mathrm{a}}$ Sum of determined constituents. 


\section{CALIFORNIA}

PALO ALTO

(Population, 25, 475)

Ownership: Municipal; supplies also about 9,750 people outside the city limits.

Total population supplied, about $35,200$.

Source: San Francisco city supply (see San Francisco) 60 percent of supply; 9 wells, 250 to $600 \mathrm{ft}$ deep, 40 percent of supply.

Treatment: Chlorination.

Raw-water storage: --

Finished-water storage: 4, 980,000 gal.

\section{ANALYSES}

(Analyses, in parts per million, by California State Dept. of Public Health)

\begin{tabular}{|c|c|c|c|c|}
\hline & $\begin{array}{c}\text { Main } \\
\text { Station } \\
\text { Well }\end{array}$ & $\begin{array}{c}\text { Mayfield } \\
\text { Well }^{\mathrm{a}}\end{array}$ & $\begin{array}{c}\text { Middlefield } \\
\text { Well }\end{array}$ & $\begin{array}{c}\text { Oregon } \\
\text { Well }\end{array}$ \\
\hline Silica $\left(\mathrm{SiO}_{2}\right) \ldots \ldots \ldots \ldots \ldots \ldots \ldots$ & -- & -- & -- & -- \\
\hline Iron $(\mathrm{Fe}) \ldots \ldots \ldots \ldots \ldots \ldots \ldots \ldots$ & .04 & .6 & 0 & .26 \\
\hline Manganese (Mn) ................. & .0 & 0 & 0 & .1 \\
\hline Calcium (Ca) $\ldots \ldots \ldots \ldots \ldots \ldots \ldots$ & 61 & 41 & 39 & 82 \\
\hline Magnesium (Mg) ............... & 14 & 12 & 11 & 22 \\
\hline Sodium (Na)....................... & 86 & 65 & 110 & 102 \\
\hline Potassium (K) ................. & -- & -- & -- & 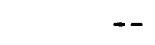 \\
\hline Carbonate $\left(\mathrm{CO}_{3}\right) \ldots \ldots \ldots \ldots \ldots$ & & 0 & 0 & 0 \\
\hline Bicarbonate $\left(\mathrm{HCO}_{3}\right) \ldots \ldots \ldots \ldots$ & 283 & 244 & 271 & 254 \\
\hline Sulfate $\left(\mathrm{SO}_{4}\right) \ldots \ldots \ldots \ldots \ldots \ldots$ & 44 & 40 & 33 & 33 \\
\hline Chloride (Cl)................. & & 35 & 90 & 170 \\
\hline Fluoride $(F)$ & .0 & 0 & 0 & .1 \\
\hline Nitrate $\left(\mathrm{NO}_{3}\right)$..................... & & 1.1 & 0 & 1.8 \\
\hline Dissolved solids ................. & 575 & 438 & 552 & 668 \\
\hline Hardness as $\mathrm{CaCO}_{\mathbf{3}}$ : & & & & \\
\hline Total $\ldots . . . . . . . . . . . . . . . . . . . . . .$. & 211 & 151 & 142 & 291 \\
\hline Noncarbonate . & 0 & 0 & 0 & 87 \\
\hline Color. & -- & -- & -- & -- \\
\hline pH ........ & 7.7 & 7.9 & 7.9 & 8.1 \\
\hline Specific conductance & & & & \\
\hline (micromhos at $25 \mathrm{C}$. ) & -- & - & -- & - \\
\hline Turbidity.................$\ldots \ldots$ & -- & -- & -- & -- \\
\hline Temperature (F.) ............... & & & & \\
\hline Date of collection................ & $\begin{array}{c}\text { Feb. } 6 \\
1951\end{array}$ & $\begin{array}{c}\text { Feb. 6, } \\
1951\end{array}$ & $\begin{array}{c}\text { Feb. 6, } \\
1951\end{array}$ & $\begin{array}{c}\text { Feb. } 6 \text {, } \\
1951\end{array}$ \\
\hline Depth (feet) & 446 & 526 & 600 & 512 \\
\hline Diameter (inches). & 14 & 14 & 14 & 14 \\
\hline Date drilled. & 1926 & 1931 & 1932 & 1930 \\
\hline Percent of supply ........ & & -- & -- & -- \\
\hline
\end{tabular}

${ }^{\text {a }}$ Also known as Park Boulevard Well. 


\section{CALIFORNIA}

PASADENA

(Population, 104, 577)

Ownership: Municipal.

Source: Colorado River distributed by Metropolitan Water District of Southern California, 62 percent of supply (see Los Angeles); 14 wells ranging in depth from 642 to 1,220 ft, 35 percent of supply; Arroyo Seco, Eaton Canyon, and Millard Streams, 3 percent of supply.

Treatment: Colorado River: softenedand filtered by Metropolitan District of Southern California (see Los Angeles). Local supplies: chlorination and ammoniation.

Rated capacity of treatment plant: --

Raw-water storage: --

Finished-water storage: 103, 000, 000 gal.

\section{ANALYSES}

(Analyses, in parts per million, by City Of Pasadena)

\begin{tabular}{|c|c|c|c|c|c|}
\hline & $\begin{array}{c}\text { Jourdan } \\
\text { Well }^{\mathbf{a}}\end{array}$ & $\begin{array}{c}\text { Garfield } \\
\text { Well }^{a}\end{array}$ & $\begin{array}{l}\text { Woodbury } \\
\text { Well }^{\mathrm{a}}\end{array}$ & $\begin{array}{c}\text { Sunset } \\
\text { Well }^{a}\end{array}$ & $\underset{\text { Well }^{\mathrm{a}}}{\text { Copelin }}$ \\
\hline 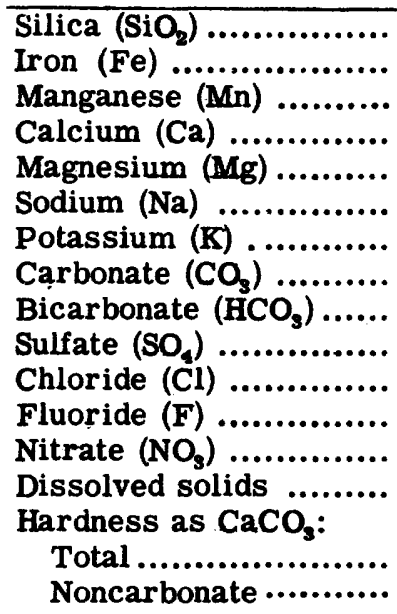 & $\begin{array}{c}30 \\
.03 \\
42 \\
11 \\
17 \\
0 \\
178 \\
13 \\
10 \\
8.7 \\
b_{219}^{--} \\
151 \\
5\end{array}$ & $\begin{array}{c}30 \\
.03 \\
20 \\
4.0 \\
25 \\
0 \\
99 \\
13 \\
10 \\
9.9 \\
9.9 \\
161 \\
65 \\
0\end{array}$ & $\begin{array}{c}26 \\
.06 \\
24 \\
3.7 \\
33 \\
0 \\
123 \\
21 \\
12 \\
b^{--}- \\
6.7 \\
74 \\
0\end{array}$ & $\begin{array}{r}30 \\
.03 \\
37 \\
11 \\
23 \\
0 \\
159 \\
18 \\
14 \\
\\
14 \\
b_{225}^{--} \\
139 \\
9\end{array}$ & $\begin{array}{c}30 \\
.03 \\
47 \\
-- \\
15 \\
26 \\
0 \\
216 \\
28 \\
13 \\
\\
6.4 \\
272 \\
179 \\
2\end{array}$ \\
\hline 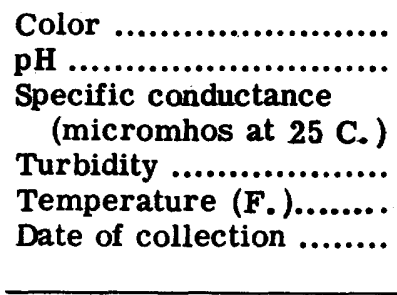 & 7.4 & $\begin{array}{l}7.7 \\
-- \\
-- \\
--\end{array}$ & $\begin{array}{l}-- \\
-- \\
-- \\
--\end{array}$ & $\begin{array}{l}-- \\
-- \\
--\end{array}$ & $\begin{array}{l}-- \\
-- \\
--\end{array}$ \\
\hline $\begin{array}{l}\text { Depth (feet) } \ldots . . . . . . . . . . \\
\text { Diameter (inches)......... } \\
\text { Date drilled .............. } \\
\text { Percent of supply ....... }\end{array}$ & $\begin{array}{r}729 \\
26 \\
1926 \\
11\end{array}$ & $\begin{array}{r}720 \\
26 \\
1921 \\
7\end{array}$ & $\begin{array}{r}1220 \\
26 \\
1930 \\
6\end{array}$ & $\begin{array}{r}751 \\
26 \\
1924 \\
6\end{array}$ & $\begin{array}{r}700 \\
26 \\
1921 \\
3\end{array}$ \\
\hline
\end{tabular}

a Average for year 1950-51.

b Sum of determined constituents. 


\section{CALIFORNIA}

PASADENA--Continued

\section{ANALYSES}

(Analyses, in parts per million, by City of Pasadena)

\begin{tabular}{|c|c|c|c|c|c|}
\hline & $\begin{array}{l}\text { Eaton } \\
\text { Canyon } \\
\text { Stream }\end{array}$ & \begin{tabular}{|l|} 
Arroyo \\
Seco \\
Stream
\end{tabular} & & $\begin{array}{l}\text { Eaton } \\
\text { Canyon } \\
\text { Stream } \\
\end{array}$ & \begin{tabular}{|l} 
Arroyo \\
Seco \\
Stream $^{\text {a }}$
\end{tabular} \\
\hline Silica $\left(\mathrm{SiO}_{2}\right)$. & & 24 & \multirow{3}{*}{$\begin{array}{c}\text { Hardness as } \mathrm{CaCO}_{3} \text {. } \\
\text { Total .................. } \\
\text { Noncarbonate...... }\end{array}$} & \multirow{3}{*}{$\begin{array}{r}175 \\
4\end{array}$} & \multirow{3}{*}{$\begin{array}{r}213 \\
5\end{array}$} \\
\hline Iron (Fe) ......... & & .07 & & & \\
\hline Manganese (Mn) & & & & & \\
\hline $\begin{array}{l}\text { Calcium (Ca) ......... } \\
\text { Magnesium (Mg)..... }\end{array}$ & & $\begin{array}{l}56 \\
18\end{array}$ & \multirow{10}{*}{ 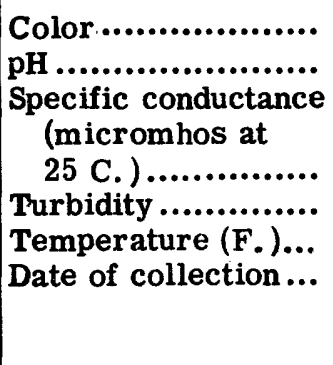 } & \multirow{4}{*}{$8 . \overline{2}$} & \multirow{3}{*}{$\overline{7.9}$} \\
\hline Sodium (Na) & \multirow{2}{*}{ - 14} & \multirow{2}{*}{26} & & & \\
\hline Potassium (K) & & & & & \\
\hline Carbonate $\left(\mathrm{CO}_{3}\right) \ldots$ & 0 & 0 & & & \\
\hline Bicarbonate $\left(\mathrm{HCO}_{3}\right)$ & 208 & 254 & & -- & \multirow{3}{*}{$\begin{array}{l}-- \\
--\end{array}$} \\
\hline Sulfate $\left(\mathrm{SO}_{4}\right) \ldots \ldots \ldots \ldots$ & & 36 & & -- & \\
\hline Chloride (Cl). & 7.2 & 13 & & -- & \\
\hline Fluoride $(F) \ldots$ & - & & & -- & -- \\
\hline Nitrate $\left(\mathrm{NO}_{3}\right) \ldots \ldots \ldots$ & $b .4$ & $b_{0} .4$ & & & \\
\hline & ${ }^{D} 224$ & ${ }^{0} 299$ & & & \\
\hline
\end{tabular}

${ }_{b}^{a}$ Average for year $1950-51$

sum of determined constituents 


\section{CALIFORNIA}

\section{POMONA}

(Population, 35, 405)

Ownership: Municipal.

Source: 12 wells (1 to 12 ) ranging in depth from 495 to $1,104 \mathrm{ft}$, and 2 tunnels

( 1 and 3). The yield of the wells is reported to range from 400 to $1,500 \mathrm{gpm}$, and to average $843 \mathrm{gai}$.

Treatment: None.

Storage: Reservoirs, 15, 000, 000 gal.

\section{ANALYSES}

(Analyses, in parts per million, by California State Dept. of Public Health)

\begin{tabular}{|c|c|c|c|c|c|}
\hline & Well 4 & Well 5 & Well 6 & Well 11 & Tunnel 3 \\
\hline 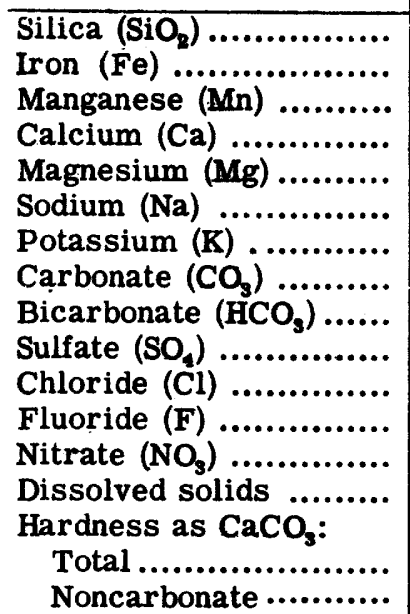 & $\begin{array}{c}20 \\
72^{--} \\
12 \\
12 \\
11 \\
214^{--} \\
29 \\
11 \\
48 \\
321 \\
231 \\
56\end{array}$ & $\begin{array}{c}19 \\
52 \\
5 .- \\
8.5 \\
20 \\
4.6 \\
-- \\
184^{--} \\
27 \\
9 \\
32 \\
270 \\
166 \\
15\end{array}$ & $\begin{array}{c}18 \\
68 \\
68 \\
7.6 \\
32 \\
4.4 \\
-- \\
170^{--} \\
64 \\
13 \\
.2 \\
80^{-2} \\
202 \\
62\end{array}$ & \begin{tabular}{|c|}
23 \\
52 \\
$5 .-$ \\
9.3 \\
$12^{--}$ \\
3.8 \\
-- \\
189 \\
20 \\
7.0 \\
.3 \\
$17^{-3}$ \\
256 \\
169 \\
14
\end{tabular} & $\begin{array}{c}16 \\
37 \\
37 \\
4.0 \\
31 \\
3.0 \\
172^{--} \\
21 \\
10 \\
13 \\
218 \\
108 \\
0\end{array}$ \\
\hline 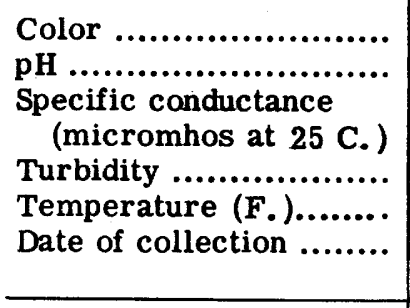 & $\begin{array}{r}0 \\
7.0 \\
495 \\
1.2 \\
-- \\
\text { May } 9, \\
1951 \\
\end{array}$ & $\begin{array}{r}0 \\
7.3 \\
403 \\
.8 \\
-- \\
\text { May } 9 \\
1951 \\
\end{array}$ & $\begin{array}{r}0 \\
7.1 \\
540 \\
.7 \\
-- \\
\text { May } 9 \\
1951 \\
\end{array}$ & $\begin{array}{r}0 \\
7.3 \\
382 \\
.6 \\
-- \\
\text { May } 9 \\
1951 \\
\end{array}$ & $\begin{array}{r}0 \\
7.3 \\
330 \\
.9 \\
-- \\
\text { May } 9, \\
1951 \\
\end{array}$ \\
\hline $\begin{array}{l}\text { Depth (feet) } \ldots . . . \ldots \ldots . . . \\
\text { Diameter (inches)......... } \\
\text { Date drilled .............. } \\
\text { Percent of supply ........ }\end{array}$ & $\begin{array}{r}537 \\
20 \\
1940 \\
--\end{array}$ & $\begin{array}{r}575 \\
20 \\
1930 \\
--\end{array}$ & $\begin{array}{r}560 \\
20 \\
1933 \\
--\end{array}$ & $\begin{array}{r}566 \\
20 \\
1947 \\
--\end{array}$ & \\
\hline
\end{tabular}




\section{CALIFORNIA}

\section{REDONDO BEACH}

(Population, 25, 226)

Ownership: California Water Service Co. ; suppliesalso Hermosa Beach and about 3,000 people outside the city limits. Total population supplied, about 40,000.

Source: 13 wells ranging in depth from 286 to $600 \mathrm{ft}$ (95 percent of supply); auxiliary supply from Colorado River distributed by the Metropolitan Water District of Southern California (5 percent of supply). (See Los Angeles.) The yield of the wells is reported to range from 180 to $1,052 \mathrm{gpm}$, and to average 557 gal.

Treatment: Chlorination of well water. Colorado River water is softened and filtered by the Metropolitan Water District of Southern California.

Storage: $3,450,000$ gal.

\section{ANALYSES}

(Analyses, in parts per million, by California Water Service Co., San Jose, Calif.)

\begin{tabular}{|c|c|c|c|c|c|}
\hline & & & & & $\begin{array}{l}\text { Well } \\
5-02\end{array}$ \\
\hline 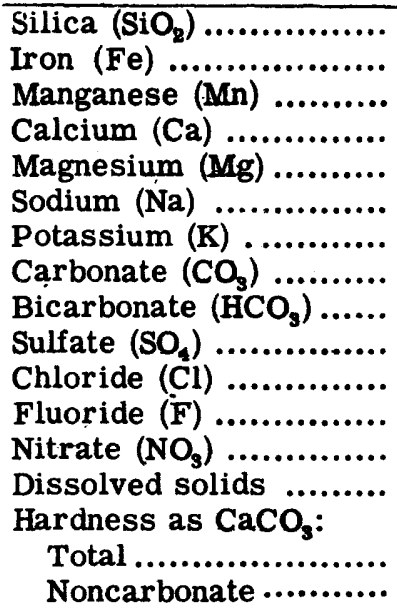 & 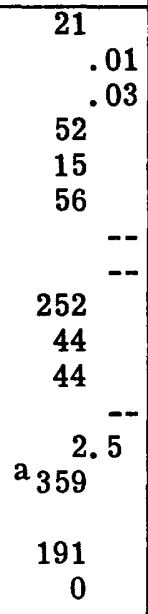 & \begin{tabular}{|c|c|}
28 & .22 \\
38 & .10 \\
24 & \\
79 & \\
& -- \\
305 & -- \\
5.3 & \\
77 & -- \\
& .6 \\
403 & \\
194 & \\
0
\end{tabular} & \begin{tabular}{ccc}
\multicolumn{1}{c}{18} & \\
& .01 \\
& .07 \\
48 & \\
15 & \\
54 & \\
& -- \\
& & -- \\
263 & \\
23 & \\
44 & \\
& & -- \\
$\mathrm{a}_{332}$ & .0
\end{tabular} & \begin{tabular}{ccc}
33 & \\
& .08 \\
& .0 \\
27 & \\
12 & \\
99 & \\
& -- \\
& -- \\
305 & \\
12 & \\
50 & \\
& & -- \\
& \multicolumn{2}{c}{.0} \\
$a_{384}$ & &
\end{tabular} & 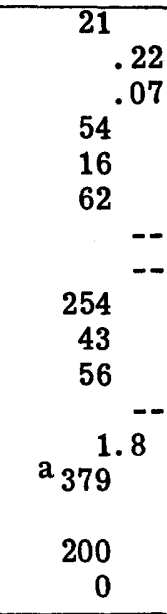 \\
\hline 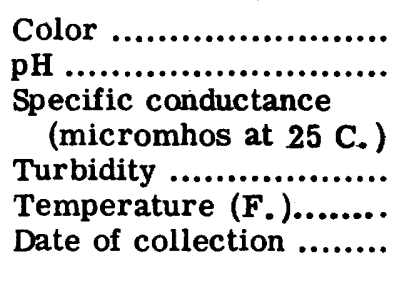 & $\begin{array}{r}612 \\
0 \\
74 \\
\text { Apr. } 13 \\
1950\end{array}$ & $\begin{array}{r}715 \\
0 \\
74 \\
\text { Feb. } 19 \\
1951\end{array}$ & $\begin{array}{r}590 \\
0 \\
72 \\
\text { Apr. } 25 \\
1950\end{array}$ & $\begin{array}{r}653 \\
0 \\
75 \\
\text { May } 10 \\
1950\end{array}$ & $\begin{array}{r}644 \\
0 \\
72 \\
\text { Apr. } 11, \\
1950\end{array}$ \\
\hline $\begin{array}{l}\text { Depth (feet) } \ldots \ldots \ldots \ldots \ldots . . \\
\text { Diameter (inches)......... } \\
\text { Date drilled ............... } \\
\text { Percent of supply ........ }\end{array}$ & $\begin{array}{r}520 \\
16 \\
1949 \\
--\end{array}$ & $\begin{array}{r}450 \\
16 \\
1944\end{array}$ & $\begin{array}{r}600 \\
16 \\
1948 \\
--\end{array}$ & $\begin{array}{r}504 \\
16 \\
1950 \\
--\end{array}$ & $\begin{array}{r}340 \\
16 \\
1908 \\
--\end{array}$ \\
\hline
\end{tabular}

${ }^{\mathrm{a}}$ Sum of determined constituents. 


\title{
CALIFORNIA
}

\author{
REDWOOD CITY \\ (Population, 25, 544)
}

Ownership: Municipal; supplies also about 4,900 people outside the city limits.

Total population supplied, about 30,400 .

Source: Hetch Hetchy aqueduct of the San Francisco supply. (See San Francisco.)

Emergency supply from local wells.

Treatment: Chlorination by City of San Francisco.

Storage: $6,420,000$ gal.

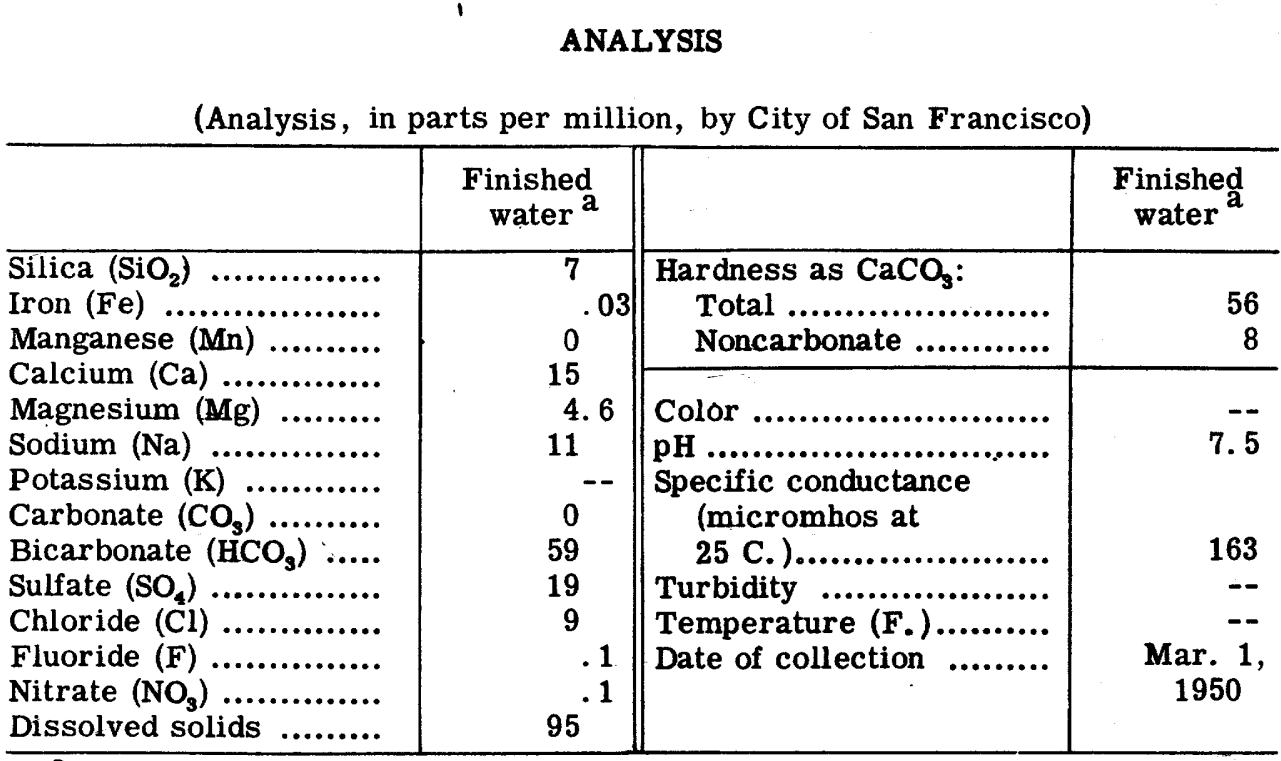

${ }^{a}$ Composite sample.

RICHMOND

(Population, 99, 545)

Ownership: East Bay Municipal Utility District. (See Oakland.) 


\section{CALIFORNIA}

\section{RIVERSIDE}

(Population, 46, 764)

Ownership: Municipal; supplies also about 1,600 people outside the city limits. Total population supplied, about 48,400 .

Source: 26 wells in the San Bernardino artesian basin. Well depths range from 300 to $1,192 \mathrm{ft}$. Most of these wells are flowing. Auxiliary supply from 5 wells in Riverside.

Treatment: None.

Storage: Reservoirs, 22, 000, 000 gal.

\section{ANALYSES}

(Analyses, in parts per million, by California State Dept. of Public Health)

\begin{tabular}{|c|c|c|c|c|c|}
\hline & $\begin{array}{l}\text { Com- } \\
\text { posite } \\
\text { sample }\end{array}$ & $\begin{array}{l}\text { Warren } \\
\text { Well } 2\end{array}$ & $\begin{array}{c}\text { Raub } \\
\text { Well } 2\end{array}$ & $\begin{array}{c}\text { Hunt } \\
\text { Wells } \\
\text { (5 wells) }\end{array}$ & $\begin{array}{l}\text { Cooley } \\
\text { Wells } \\
\text { (9 wells) }\end{array}$ \\
\hline Silica $\left(\mathrm{SiO}_{2}\right) \ldots \ldots \ldots \ldots \ldots$ & 16 & 17 & 15 & 18 & 18 \\
\hline Iron $(\mathrm{Fe}) \ldots \ldots \ldots \ldots \ldots \ldots$ & -- & -- & .01 & -- & -- \\
\hline Manganese (Mn) ........... & -- & -- & -- & -- & -- \\
\hline Calcium (Ca) .............. & 27 & 33 & 28 & 26 & 35 \\
\hline Magnesium (Mg) .......... & 4 & 4.4 & 2.7 & 4.9 & 7.0 \\
\hline Sodium (Na) ................ & 50 & 16 & 57 & 51 & 31 \\
\hline Potassium (K) . ........... & -- & 3.2 & 5.6 & 3.6 & 4.4 \\
\hline Carbonate $\left(\mathrm{CO}_{3}\right) \ldots \ldots \ldots \ldots$ & 0 & - & & -- & -- \\
\hline Bicarbonate $\left(\mathrm{HCO}_{3}\right) \ldots \ldots$ & 162 & 144 & 151 & 162 & \\
\hline Sulfate $\left(\mathrm{SO}_{4}\right) \ldots \ldots \ldots \ldots \ldots$ & 34 & 16 & 44 & 31 & 28 \\
\hline Chloride (Cl) ............. & 18 & 5.5 & 27 & 24 & 8.0 \\
\hline 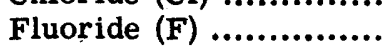 & .4 & .5 & .8 & .4 & .2 \\
\hline Nitrate $\left(\mathrm{NO}_{3}\right)$. ................. & & 1.1 & 1.4 & 1.9 & 1.3 \\
\hline Dissolved solids ......... & $b_{229}$ & 170 & 246 & 262 & 216 \\
\hline Hardness as $\mathrm{CaCO}_{3}$ : & & & & & \\
\hline Total ....................... & 84 & 102 & 80 & 85 & 116 \\
\hline Noncarbonate ............ & $\mathbf{0}$ & 0 & $\mathbf{0}$ & 0 & 0 \\
\hline Color ... & -- & $\mathbf{0}$ & 0 & 0 & 0 \\
\hline pH ........ & -- & 7.2 & 7.4 & 7.7 & 7.4 \\
\hline Specific conductance & & & & & \\
\hline (micromhos at $25 \mathrm{C}$. ) & 350 & 266 & 208 & 374 & 355 \\
\hline Turbidity & -- & 1.2 & .9 & .9 & .5 \\
\hline Temperature (F. )........ & -- & & -- & & -- \\
\hline Date of collection ......... & $\begin{array}{c}\text { Nov. } 17, \\
1950\end{array}$ & $\begin{array}{c}\text { May 8, } \\
1951\end{array}$ & $\begin{array}{c}\text { May } 8 \\
1951\end{array}$ & $\begin{array}{l}\text { May 8, } \\
1951\end{array}$ & $\begin{array}{c}\text { May 8, } \\
1951\end{array}$ \\
\hline \multirow{3}{*}{\multicolumn{2}{|c|}{ 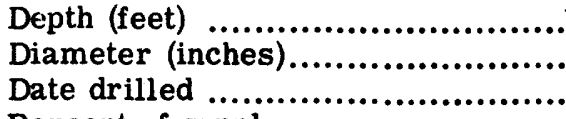 }} & 1,137 & 1,192 & $409-868$ & $520-1,138$ \\
\hline & & 20 & 20 & $10-20$ & $10-20$ \\
\hline & & 1930 & 1931 & $1912-46$ & $1899-1947$ \\
\hline \multicolumn{2}{|c|}{ Percent of supply ........................ } & -- & -- & -- & -- \\
\hline
\end{tabular}




\section{CALIFORNIA}

\section{SACRAMENTO}

(Population, 137, 572)

Ownership: Municipal; supplies also about 13,500 people outside the city limits. Total population supplied, about 151,100. Southern California Water Co. supplies about 4 percent of the population.

Source: Sacramento River ( 96 percent of supply); 20 wells (owned by Southern California Water Co.), 4 percent of the supply.

Treatment: Sacramento River: coagulation with alum, sedimentation, rapid sand filtration, and chlorination. Wells: none.

Rated capacity of treatment plant: $64,000,000 \mathrm{gpd}$.

Raw-water storage: None.

Finished-water storage: 14,000,000 gal.

The intake in the Sacramento River is just below the mouth of the American River. Analytical data indicate that at times the water withdrawn represents chiefly American River water.

\section{ANALYSES}

(Analyses, in parts per million, by U. S. Geological Survey)

\begin{tabular}{|c|c|c|c|c|c|}
\hline & $\begin{array}{c}\text { Raw } \\
\text { water a }\end{array}$ & $\begin{array}{c}\text { Finished } \\
\text { water a }\end{array}$ & & $\underset{\text { water a }}{\text { Raw }}$ & $\begin{array}{r}\text { Finished } \\
\text { water a }\end{array}$ \\
\hline $\begin{array}{l}\text { Silica }\left(\mathrm{SiO}_{2}\right) \ldots \ldots \ldots \ldots \\
\text { Iron }(\mathrm{Fe}) \ldots \ldots \ldots \ldots . \\
\text { Manganese }(\mathrm{Mn}) \ldots . . .\end{array}$ & $\begin{array}{r}9.2 \\
.0 \\
--\end{array}$ & $\begin{array}{l}9.1 \\
.0 \\
--\end{array}$ & $\begin{array}{c}\text { Hardness as } \mathrm{CaCO}_{\mathbf{3}} \text { : } \\
\text { Total ................... } \\
\text { Noncarbonate..... }\end{array}$ & $\begin{array}{r}20 \\
0\end{array}$ & $\begin{array}{r}21 \\
6\end{array}$ \\
\hline $\begin{array}{l}\text { Calcium (Ca) .......... } \\
\text { Magnesium (Mg)..... }\end{array}$ & $\begin{array}{l}4.6 \\
2.0\end{array}$ & $\begin{array}{l}5.0 \\
2.0\end{array}$ & Color & 15 & $\mathbf{0}$ \\
\hline Sodium $(\mathrm{Na})$ & 1.5 & 1.5 & pH $\ldots \ldots \ldots \ldots \ldots \ldots \ldots \ldots$ & 7.2 & 6.7 \\
\hline $\begin{array}{l}\text { Potassium }(\mathbf{K}) \\
\text { Carbonate }\left(\mathrm{CO}_{2}\right)\end{array}$ & $0^{.6}$ & .9 & $\begin{array}{l}\text { Specific conductance } \\
\text { (micromhos at }\end{array}$ & & \\
\hline Bicarbonate ( $\left.\mathrm{HCO}_{3}\right)$ & 23 & 18 & 25 C. $) \ldots \ldots \ldots \ldots$ & 46.8 & 54.6 \\
\hline Sulfate $\left(\mathrm{SO}_{4}\right) \ldots \ldots \ldots . .$. & 1.7 & 7.6 & Turbidity.. & -- & -- \\
\hline Chloride (Cl) .. & .9 & 1.4 & Temperature (F.).. & 54 & 58 \\
\hline Fluoride $(F)$ & .0 & .0 & Date of collection... & June 6, & June 6, \\
\hline Nitrate $\left(\mathrm{NO}_{3}\right) \ldots . . . .$. & .6 & .4 & & 1952 & 1952 \\
\hline Dissolved solids..... & 36 & 37 & & & \\
\hline
\end{tabular}

\footnotetext{
${ }^{\mathrm{a}}$ Sacramento River.
} 


\section{CALIFORNIA}

SAN BERNARDINO

(Population, 63, 058)

Ownership: Municipal.

Source: 28 wells ranging in depth from 40 to $1,408 \mathrm{ft}$ (82 percent of supply), and Lytle Creek (18 percent of supply). The yield of the wells is reported to range from 125 to $2,900 \mathrm{gpm}$, and to average 1,298 gal (data on 21 wells).

Treatment: Chlorination of creek water; well water not treated.

Storage: Reservoirs, 37, 690, 000 gal.

\section{ANALYSES}

(Analyses, in parts per million, by California State Dept. of Public Health)

\begin{tabular}{|c|c|c|c|c|c|}
\hline & $\begin{array}{c}\text { Antil } \\
\text { Well 5 }\end{array}$ & $\begin{array}{l}\text { 17th St. \& } \\
\text { Sierra Way } \\
\text { Well }\end{array}$ & $\begin{array}{l}\text { Hanford } \\
\text { Well } 1 \\
\end{array}$ & $\begin{array}{c}\text { Perris } \\
\text { Hill } \\
\text { Well 3 } \\
\end{array}$ & $\begin{array}{l}\text { Lytle } \\
\text { Creek a }^{\text {a }}\end{array}$ \\
\hline Silica $\left(\mathrm{SiO}_{2}\right) \ldots \ldots \ldots \ldots \ldots$ & - & 14 & 9 & 15 & -- \\
\hline Iron $(\mathrm{Fe})$ & -- & .03 & -- & -- & 0 \\
\hline Manganese (Mn) ........... & -- & -- & -- & -- & -- \\
\hline Calcium (Ca) .............. & 24 & 52 & 47 & 31 & 52 \\
\hline Magnesium (Mg) ........... & 1.8 & 9.5 & 7.7 & 6.1 & 6 \\
\hline Sodium (Na) ............... & 44 & 12 & 17 & 32 & 41 \\
\hline Potassium (K) ............ & 2.2 & 4.0 & 4.0 & .3 & -- \\
\hline Carbonate $\left(\mathrm{CO}_{3}\right) \ldots \ldots \ldots . .$. & -- & -- & - & -- & 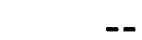 \\
\hline Bicarbonate $\left(\mathrm{HCO}_{3}\right) \ldots . .$. & 127 & 210 & 188 & 129 & 244 \\
\hline Sulfate $\left(\mathrm{SO}_{4}\right) \ldots \ldots \ldots \ldots \ldots$ & 42 & 13 & 30 & 27 & 27 \\
\hline Chloride (Cl) .............. & 7.4 & 4.8 & 10 & 18 & 12 \\
\hline Fluoride (F) ............... & .4 & .4 & .4 & & -- \\
\hline Nitrate $\left(\mathrm{NO}_{3}\right) \ldots \ldots \ldots \ldots \ldots$ & 1.3 & 5.3 & 3.7 & 3.5 & \\
\hline Dissolved solids .......... & 232 & 244 & 240 & ${ }^{b_{197}}$ & $\mathrm{~b}_{258}$ \\
\hline Hardness as $\mathrm{CaCO}_{3}$ : & & & & & \\
\hline 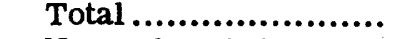 & 67 & 169 & 149 & 102 & 154 \\
\hline Noncarbonate :........... & 0 & 0 & 0 & 0 & 0 \\
\hline Color .... & 0 & 0 & 0 & 0 & -- \\
\hline pH .................... & 8.8 & 8.5 & 8.4 & 8.0 & 8.0 \\
\hline (micromhos at $25 \mathrm{C}$.) & 319 & 339 & 325 & 352 & - \\
\hline Turbidity .................... & 1.1 & 1.1 & 1.0 & 3.5 & - \\
\hline Temperature (F.)........ & - & - & - & - & -- \\
\hline Date of collection ......... & $\underset{1951}{\text { Apr. } 18,}$ & $\begin{array}{c}\text { Apr. } 18, \\
1951\end{array}$ & $\underset{1951}{\text { Apr. } 18,}$ & $\underset{1951}{\text { Apr. } 18,}$ & 1940 \\
\hline Depth (feet) ........ & 1,408 & & & & \\
\hline Diameter (inches)......... & - & 20 & 16 & 20 & \\
\hline Date drilled .. & 1929 & 1948 & 1920 & 1930 & \\
\hline f supply ......... & -- & -- & -- & -- & \\
\hline
\end{tabular}

${ }^{a}$ Special Bulletin No. 63, California Water Supply Statistics, California State Dept. of Health.

${ }^{b}$ Sum of determined constituents. 


\section{CALIFORNIA}

SAN DIEGO

(Population, 334, 387)

Ownership: Municipal; supplies San Dieguito Irrigation District, Santa Fe Irrigation District, La Jolla, Del Mar, and part of Coronado. Total population supplied, about 342,000 .

Source: San Diego River system (62 percent of supply), Cottonwood-Otay system (32 percent of supply), and San Dieguito system (6 percent of supply). Emergency supplies from Lake Henshaw, Sweetwater River water furnished by California Water and Telephone Co., and Colorado River through the San Diego aqueduct of the Metropolitan Water District of Southern California. (See Los Angeles.)

Treatment: Alvarado plant: prechlorination, coagulation with ferric sulfate, partial softening with lime, settling, recarbonation, rapid sand filtration, postchlorination, and adjustment of $\mathbf{p H}$ with lime. Lower Otay plant: pressure filtration and chlorination. Torrey Pines plant: pressure filtration and chlorination. There are several stations for chlorination only. Fluoridation is scheduled to begin at the Alvarado plant within the first few months of 1952.

Rated capacity of treatment plant: Alvarado plant: 66,000,000 gpd. Lower Otay plant: 16,000,000 gpd. Torrey Pines plant: 3, 000,000 gpd.

Raw-water storage: $130,076,000,000$ gal.

Finished-water storage: $136,000,000$ gal.

The percentages of supply shown above are the "normal" percentages. Considerable quantities of Colorado River water have been used for the past few dry years, due to extremely low production of local supplies. For the year ending June 30, 1951, almost 90 percent of the supply came from the Colorado River, distributed through the San Dieguito system and San Vicente Reservoir.

The San Diego River system includes water from the San Diego River and tributaries, and is stored in El Capitan and San Vicente Reservoirs. However, most of the water received at present in San Vicente Reservoir is Colorado River water through the San Diego Aqueduct. Water from the San Diego River system is treated at the Alvarado Plant.

The Cottonwood-Otay system includes water from Buchman and La Posta Creeks (tributaries of Cottonwood Creek) stored in Morena Reservoir, Cottonwood and Pine Valley Creeks stored in Barrett Reservoir, and Dulzura Creek stored in Lower Otay Reservoir. All Cottonwood-Otay sources eventually reach Lower Otay Reservoir.

The San Dieguito River system stores water from the San Dieguito River in Lake Hodges and San Dieguito Reservoir. Water in this system is treated at Torrey Pines plant. 


\section{CALIFORNIA}

\section{ANALYSES}

(Analyses, in parts per million, by California State Dept. of Public Health)

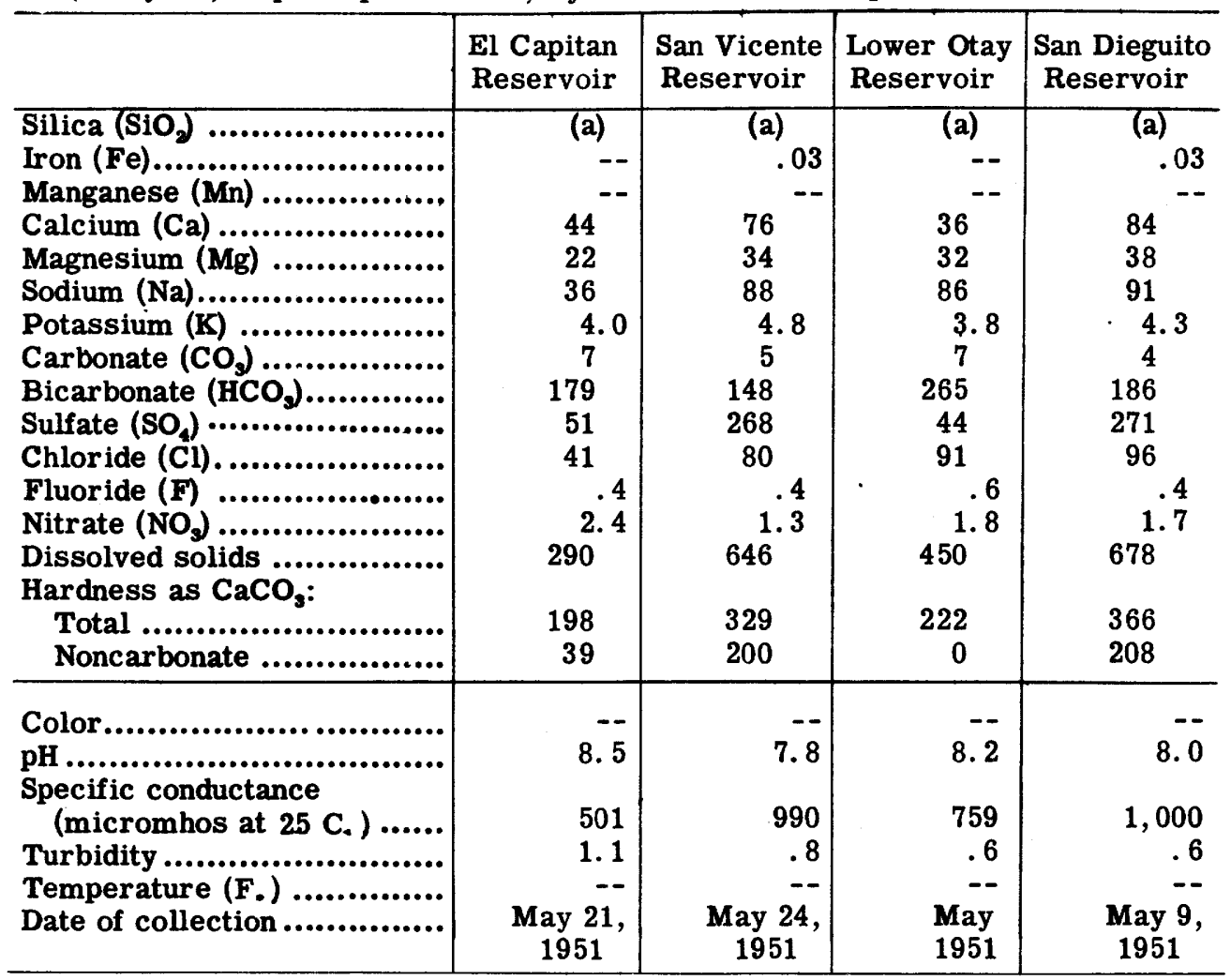

${ }^{a}$ Mean silica values obtained from quarterly analyses for the fiscal year 1950 51 by Carl Wilson, Los Angeles, are as follows: El Capitan Reservoir, 21 ppm; San Vicente Reservoir, 9 ppm; Lower Otay Reservoir, 10 ppm; San Dieguito Reservoir, 17 ppm. 


\title{
CALIFORNIA
}

\author{
SAN FRANCISCO \\ (Population, 775, 357)
}

Ownership: Municipal; furnishes the entire supply for Belmont, Belmont Water District, Burlingame, Moffett Field, Redwood City, San Carlos, San Mateo, and Sunol; furnishes part of the supply for Alameda County Water District, Atherton, Daly City, Menlo Park, Millbrae, Palo Alto, and South San Francisco. Total population supplied, about 975,000 .

Source: Hetch Hetchy system (60 percent of supply), Alameda system (approximately 30 percent of supply), Peninsula system (approximately 10 percent of supply). Emergency supplies from Sunset wells system and from Lake Merced, within San Francisco.

Treatment: Chlorination, copper sulfate for algae control in open reservoirs. Aeration of water from Calaveras Reservoir. Fluoridation authorized in November 1951.

Raw-water storage: Storage reservoirs, total capacity $187,630,000,000$ gal.

Finished-water storage: Distribution reservoirs, total capacity 315,600, 000 gal; elevated tanks, total capacity $2,780,000$ gal.

The Hetch Hetchy system includes the Tuolumne River impounded in Hetch Hetchy Reservoir, Eleanor Creek impounded in Lake Eleanor, and Cherry River. These waters are combined at Early Intake, 155 miles east of San Francisco, where they enter the aqueduct leading to San Francisco.

Hetch Hetchy Reservoir has a storage capacity of 117,300, 000, 000 gal, Lake Eleanor a storage capacity of $9,000,000,000$ gal, while the present capacity of the aqueduct is about $92,000,000 \mathrm{gpd}$. Under construction (1951) is a dam on Cherry River which will create a new reservoir with a capacity of $86,000,000,000 \mathrm{gal}$. Also under construction is a second section of aqueduct across San Joaquin Valley which will increase the capacity of the aqueduct to $140,000,000 \mathrm{gpd}$.

The Alameda sources lie on the east side of San Francisco Bay within the drainage area of Alameda Creek. The chief source is Calaveras Reservoir, which impounds Calaveras Creek and Arroyo Hondo, and water diverted from upper Alameda Creek through a tunnel. Calaveras Reservoir has a storage capacity of $31,550,000,000$ gal, and its water flows by gravity to enter the Hetch Hetchy aqueduct. During dry years water is also obtained from two underground sources: Sunol filter galleries on Alameda Creek and wells in the vicinity of Pleasanton. When these sources are used, the water is pumped into the Hetch Hetchy aqueduct near the Bay Crossing Division.

The Peninsula system includes chiefly three reservoirs: Crystal Springs, Pilarcitos, and San Andres. These reservoirs catch and store the local runoff; also Crystal Springs is the terminal reservoir for the Hetch Hetchy aqueduct, which includes all of the Hetch Hetchy and Alameda sources. The storage capacity of each reservoir is as follows: Crystal Springs, 22, 580,000, 000 gal; San Andres, 6, 190, 000, 000 gal; Pilarcitos, 1, 010, 000, 000 gal. Water from Pilarcitos Reservoir is released to San Andres Reservoir. Water from Crystal Springs and San Andres Reservoirs is supplied to a number of distribution reservoirs throughout the city. Crystal Spring lines supply downtown, commercial, waterfront areas of the city, and peninsula communities as far south as San Carlos. San Andres lines furnish water to residential areas of San Francisco. Bay Crossing lines (Hetch Hetchy aqueduct) supply peninsula communities south of San Carlos and some communities in Alameda County. 


\section{CALIFORNIA}

\section{ANALYSES}

(Analyses, in parts per million, by City of San Francisco)

\begin{tabular}{|c|c|c|c|c|c|}
\hline & $\begin{array}{c}\text { Hetch } \\
\text { Hetchy } \\
\text { Reservoir }\end{array}$ & $\begin{array}{l}\text { Calaveras } \\
\text { Reservoir }\end{array}$ & $\begin{array}{l}\text { Pilarcitos } \\
\text { Reservoir }\end{array}$ & $\begin{array}{l}\text { Sunol } \\
\text { Galleries }\end{array}$ & $\begin{array}{c}\text { Pleasanton } \\
\text { Wells }\end{array}$ \\
\hline 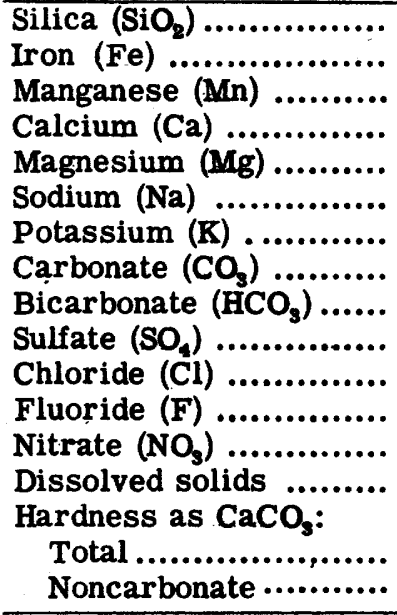 & 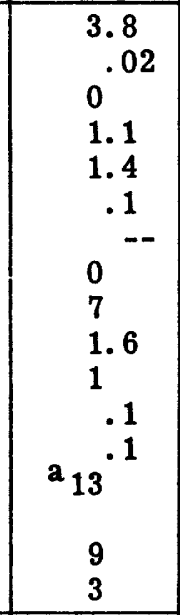 & $\begin{array}{c}5.0 \\
.02 \\
0 \\
25 \\
8.8 \\
8.8 \\
0^{--} \\
105 \\
17 \\
7 \\
.1 \\
.2 \\
a_{124} \\
99 \\
13 \\
\end{array}$ & \begin{tabular}{c}
3.7 \\
.02 \\
0 \\
15 \\
5.1 \\
14 \\
\multicolumn{1}{c}{--} \\
0 \\
70 \\
6.5 \\
20 \\
.0 \\
.1 \\
99 \\
59 \\
1 \\
\end{tabular} & 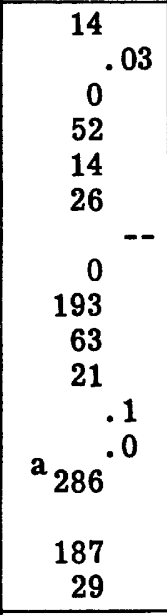 & $\begin{array}{l}11.02 \\
0 \\
63 \\
29 \\
28 \\
2^{0} \\
283 \\
47 \\
30 \\
.1 \\
1.0 \\
348 \\
275 \\
44 \\
\end{array}$ \\
\hline 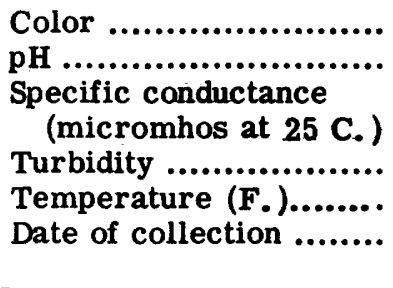 & $\begin{array}{r}-- \\
6.4 \\
19.7 \\
-- \\
-- \\
\text { June } 1949\end{array}$ & $\begin{array}{r}7 . \overline{7} \\
225 \\
-- \\
-- \\
\text { June } 1949\end{array}$ & $\begin{array}{r}7.5 \\
175 \\
-- \\
-\bar{J} \\
\text { June } 1949\end{array}$ & $\begin{array}{r}7.9 \\
466 \\
-- \\
-- \\
1948\end{array}$ & $\begin{array}{r}8.0 \\
606 \\
-- \\
-- \\
\text { January } \\
1949\end{array}$ \\
\hline
\end{tabular}

${ }^{\text {a }}$ Sum of determined constituents. 


\section{CALIFORNIA}

SAN FRANCISCO--Continued

\begin{tabular}{|c|c|c|c|}
\hline (Analyses, in parts $p$ & $\begin{array}{l}\text { ANALYSES } \\
\text { million, by C }\end{array}$ & ity of San Franci & sco) \\
\hline & $\begin{array}{c}\text { Crystal Springs } \\
\text { lines }\end{array}$ & $\begin{array}{c}\text { San Andres } \\
\text { lines }\end{array}$ & $\begin{array}{l}\text { Bay Crossing } \\
\text { lines }\end{array}$ \\
\hline 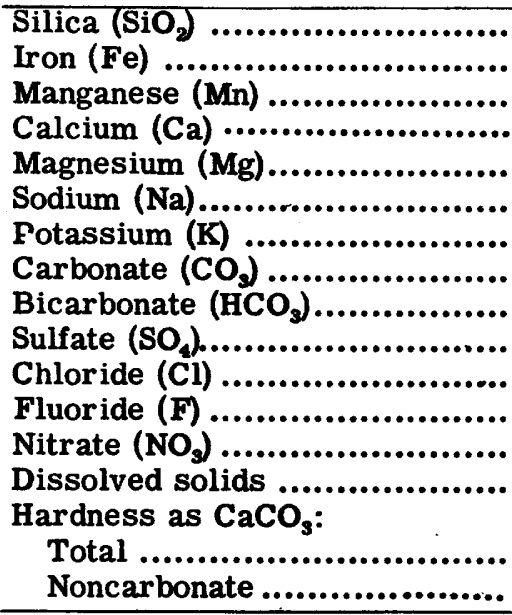 & 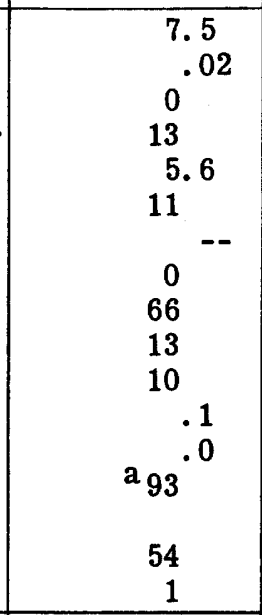 & $\begin{array}{c}7.0 \\
.01 \\
0 \\
14 \\
6.2 \\
12 \\
0^{--} \\
71 \\
12 \\
15 \\
.0 \\
a_{102} \\
58 \\
2 \\
\end{array}$ & \begin{tabular}{|l}
3.0 \\
.05 \\
0 \\
7.5 \\
3.4 \\
5.3 \\
0 \\
0 \\
34 \\
8.7 \\
5 \\
.0 \\
.0 \\
$50^{-0}$ \\
33 \\
5
\end{tabular} \\
\hline 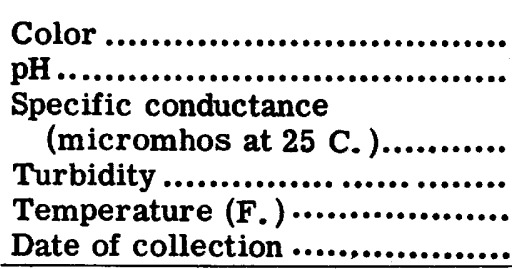 & $\begin{array}{r}1 \overline{ } \\
7.9 \\
152 \\
-- \\
-- \\
\text { Apr. 17, } 1951 \\
\end{array}$ & $\begin{array}{r}7.8 \\
170 \\
-- \\
-- \\
\text { Apr. } 17,1951\end{array}$ & $\begin{array}{c}7.3 \\
85 \\
-- \\
\text { Aug. } 18, \overline{1950} \\
\end{array}$ \\
\hline
\end{tabular}

${ }^{\mathrm{a}}$ Sum of determined constituents. 


\title{
CALIFORNIA
}

\author{
SAN JOSE \\ (Population, 95, 280)
}

Ownership: San Jose Water Works (private); supplies also Los Gatos and about 65,300 people outside the city limits. Total population supplied, about 165,500 . Source: 41 wells ranging in depth from 185 to $1,535 \mathrm{ft}$ (60 percent of supply); Los Gatos and Saratoga Creeks (40 percent of supply).

Treatment: Wells: chlorination for some, others untreated. Creeks: chlorination, occasional use of chlorine dioxide and ammonia.

Rated capacity of treatment plant:

Raw-water storage: Impounding reservoirs for streams, 2, 485, 000, 000 gal.

Finished-water storage: Distribution reservoirs, 42, 800, 000 gal.

\section{ANALYSIS}

(Analysis, in parts per million, by California Water Service Co.)

\begin{tabular}{|c|c|c|c|}
\hline & $\begin{array}{c}\text { Composite } \\
\text { water }^{\mathrm{a}}\end{array}$ & & $\begin{array}{c}\text { Composite } \\
\text { water }^{\mathrm{a}}\end{array}$ \\
\hline \multirow{2}{*}{ 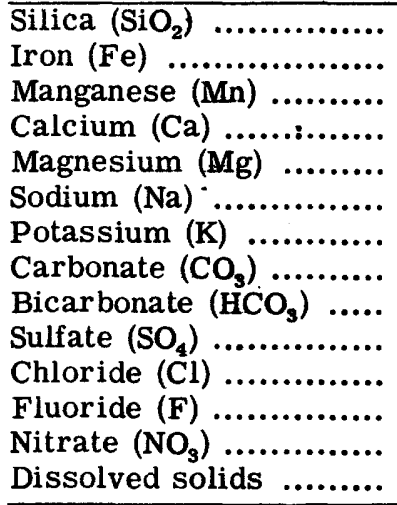 } & \multirow{2}{*}{$\begin{array}{l}21 \\
.04 \\
.00 \\
42 \\
21 \\
29 \\
1 \\
0 \\
222 \\
41 \\
16 \\
.1 \\
6.8 \\
88\end{array}$} & $\begin{array}{l}\text { Hardness as } \mathrm{CaCO}_{\mathbf{3}}: \\
\text { Total ....................... } \\
\text { Noncarbonate ........... }\end{array}$ & $\begin{array}{r}194 \\
8 \\
\end{array}$ \\
\hline & & 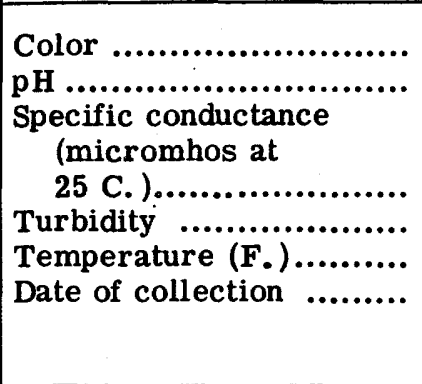 & $\begin{array}{r}475 \\
-- \\
--\end{array}$ \\
\hline \multicolumn{3}{|c|}{ 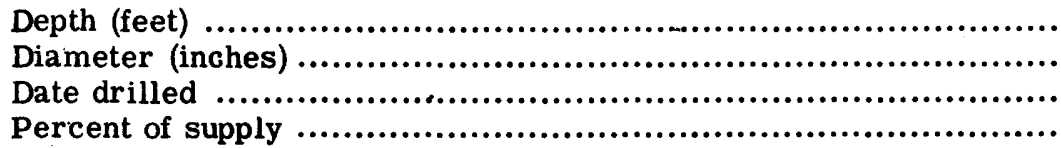 } & $\overline{100}$ \\
\hline
\end{tabular}

a Mean for 1950.

b Sum of determined constituents.

SAN LEANDRO

(Population, 27, 542)

Ownership: East Bay Municipal Utility District. (See Oakland.)

SAN MATEO

(Population, 41, 782)

Ownership: California Water Service Co.

Source: Crystal Springs Reservoir (see San Francisco). Emergency supply from 10 wells, ranging in depth from 237 to. $445 \mathrm{ft}$.

Treatment: Chlorination of water from Crystal Springs Reservoir by City of San Francisco; softening by lime-soda process, and chlorination of well water, when used.

Finished-water storage: $5,540,000$ gal. 


\title{
CALIFORNIA
}

\author{
SANTA ANA \\ (Population, 45, 533)
}

Ownership: Municipal.

Source: Colorado River distributed by the Metropolitan Water District of Southern

California , 83 percent of supply. (See Los Angeles.) Six wells (7, 12 to 16), $960,466,960,978,1,140$, and $1,050 \mathrm{ft}$ deep, 17 percent of supply.

Treatment: Chlorination of well water; Colorado River water treated by Metropolitan Water District of Southern California. (See Los Angeles.)

Raw-water storage: --

Finished-water storage: 2, 060, 000 gal.

\begin{tabular}{|c|c|c|c|c|}
\hline & Well 13 & Well 14 & Well 15 & Well 16 \\
\hline 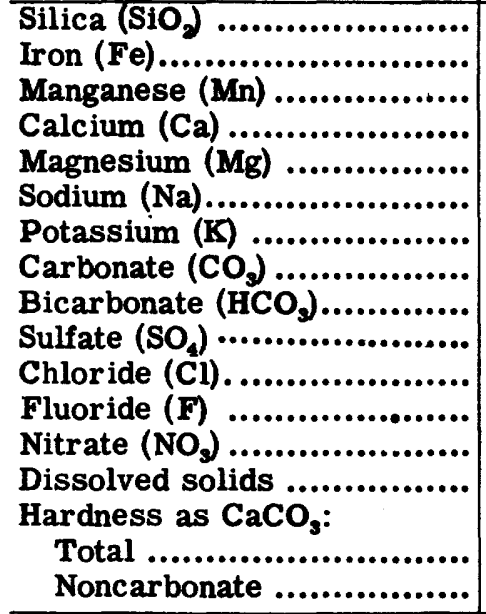 & \begin{tabular}{|l}
18 \\
.05 \\
$51^{.00}$ \\
18 \\
45 \\
1.9 \\
$204^{--}$ \\
93 \\
39 \\
. .3 \\
16 \\
$a_{383}$ \\
203 \\
36
\end{tabular} & $\begin{array}{l}16 \\
.07 \\
63 \\
.00 \\
16 \\
43 \\
2.3 \\
{ }^{-0-} \\
230 \\
74 \\
54 \\
.5 \\
12 \\
394 \\
225 \\
36\end{array}$ & \begin{tabular}{|c|}
18 \\
.00 \\
.00 \\
50 \\
19 \\
43 \\
1.7 \\
207 \\
90 \\
35 \\
.3 \\
4.4 \\
$a_{364}$ \\
205 \\
35
\end{tabular} & $\begin{array}{c}15 \\
.00 \\
.00 \\
67 \\
20 \\
43 \\
1.3 \\
208 \\
83 \\
53 \\
2 . \\
21 \\
406 \\
250 \\
79\end{array}$ \\
\hline 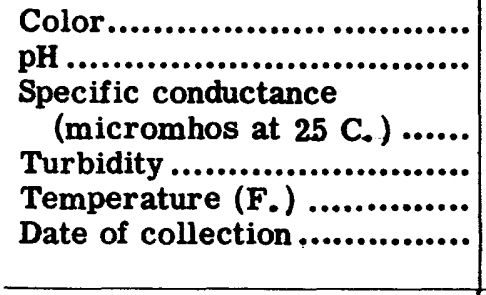 & $\begin{array}{c}7.5 \\
645 \\
<1 \\
-- \\
\text { Sept. } 25, \\
1951\end{array}$ & $\begin{array}{r}7.4 \\
708 \\
<1 \\
-- \\
\text { Sept. } 25, \\
1951 \\
\end{array}$ & $\begin{array}{c}7.5 \\
625 \\
<1 \\
-- \\
\text { Sept. } 26 \\
1951\end{array}$ & $\begin{array}{l}7.5 \\
685 \\
<1 \\
-- \\
\text { Sept. } 25 \\
1951\end{array}$ \\
\hline 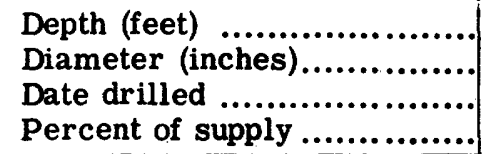 & $\begin{array}{r}960 \\
18-16 \\
1925 \\
--\end{array}$ & $\begin{array}{r}978 \\
20-16 \\
1932 \\
--\end{array}$ & $\begin{array}{r}1,140 \\
26-16 \\
1929\end{array}$ & $\begin{array}{r}1,050 \\
20-12 \\
1927\end{array}$ \\
\hline
\end{tabular}

${ }^{a_{\text {Sum }}}$ of determined constituents. 


\title{
CALIFORNIA
}

\author{
SANTA BARBARA \\ (Fopulation, 44, 913)
}

Ownership: Municipal; supplies also about 400 people. outside the city limits.

Total population supplied, about 45,300 .

Source: Santa Inez River impounded in Gibraltar Reservoir, 60 percent of supply; 9 wells ranging in depth from 473 to $946 \mathrm{ft}, 40$ percent of supply. The yield of the wells is reported to range from 250 to $800 \mathrm{gpm}$. Auxiliary supply, 5 wells. There is an emergency connection with a private water company.

Treatment: Santa Inez River water: prechlorination, lime-soda softening, recarbonation, coagulation with alum, rapid sand filtration, and postchlorination.

Well water: chlorination.

Rated capacity of treatment plant: 6, 000,000 gpd.

Raw-water storage: Gibraltar Reservoir, approximately 4, 700, 000, 000 gal.

Finished-water storage: Reservoirs, 64,000, 000 gal.

Santa Inez River is supplemented when available by water from Tecolote Tunnel.

When water from this source is used no softening is done. Sheffield Reservoir serves to distribute both Santa Inez River and Tecolote Tunnel water.

\section{ANALYYSS}

(Analyses, in parts per million, by Pomeroy \& Associates, Pasadena, Calif.)

\begin{tabular}{|c|c|c|c|c|}
\hline & $\begin{array}{c}\text { Yanonali } \\
\text { Well } 1 \\
\end{array}$ & $\begin{array}{r}\text { Soledad } \\
\text { Well } 1 \\
\end{array}$ & $\begin{array}{c}\text { De La } \\
\text { Guerra } \\
\text { Well } 1\end{array}$ & $\begin{array}{l}\text { De La } \\
\text { Guerra } \\
\text { Well } 3 \\
\end{array}$ \\
\hline Silica $\left(\mathrm{SiO}_{2}\right) \ldots \ldots \ldots \ldots \ldots \ldots \ldots$ & 32 & 32 & 38 & 28 \\
\hline Iron $(\mathrm{Fe}) \ldots . . . . . . \ldots \ldots \ldots \ldots . . . . .$. & .1 & .0 & .0 & .0 \\
\hline Manganese $(\mathrm{Mn}) . . .$. & -- & -- & - & \\
\hline Calcium (Ca) ..................... & 70 & 88 & 103 & 81 \\
\hline Magnesium (Mg) .... & 23 & 24 & 36 & 23 \\
\hline Sodium $(\mathrm{Na}) . . . . . . . . .$. & 53 & 75 & 54 & 42 \\
\hline Potassium (K) .................. & 1.7 & 2 & 2.0 & 1.4 \\
\hline Carbonate $\left(\mathrm{CO}_{3}\right), \ldots \ldots \ldots . .$. & -- & & -- & -- \\
\hline Bicarbonate (HCO).............. & 251 & 301 & 249 & 252 \\
\hline Sulfate $\left(\mathrm{SO}_{4}\right) \ldots \ldots \ldots \ldots \ldots \ldots$ & 134 & 135 & 106 & 125 \\
\hline Chloride (Cl) $\ldots . . . \ldots \ldots \ldots \ldots \ldots$ & 27 & 65 & 131 & 30 \\
\hline Fluoride (F) ..................... & .4 & .3 & .4 & .3 \\
\hline 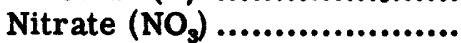 & & 2 & 40 & 4 \\
\hline Dissolved solids .... & $a_{465}$ & $a_{572}$ & $\mathbf{a}_{633}$ & $a_{459}$ \\
\hline Hardness as $\mathrm{CaCO}_{3}$ : & & & & \\
\hline Total $\ldots \ldots \ldots \ldots \ldots \ldots \ldots \ldots$ & 268 & 319 & 408 & 295 \\
\hline Noncarbonate ................. & 64 & 72 & 201 & 90 \\
\hline Color.. & & -- & -- & - \\
\hline pH ... & 6.9 & 7.2 & 6.7 & 7.0 \\
\hline Specific conductance & & & & \\
\hline ahos a & -- & -- & -- & -- \\
\hline Turbidity .............. & - & -- & -- & - \\
\hline Temperature (F.) .............. & & & & -- \\
\hline Date of collection.... & $\begin{array}{c}\text { Aug. 29, } \\
1951\end{array}$ & $\underset{1951}{\text { Aug. } 29,}$ & $\underset{1951}{\text { Aug. 29, }}$ & $\underset{1951}{\text { Aug. 29, }}$ \\
\hline
\end{tabular}

a Sum of determined constituents. 
CALIFORNIA

SANT A BARBARA--Continued

\begin{tabular}{|c|c|c|c|c|}
\hline & $\begin{array}{l}\text { Yanonali } \\
\text { Well } 1\end{array}$ & $\begin{array}{l}\text { Soledad } \\
\text { Well } 1\end{array}$ & $\begin{array}{r}\text { De La } \\
\text { Guerra } \\
\text { Well 1 }\end{array}$ & $\begin{array}{c}\text { De La } \\
\text { Guerra } \\
\text { Well } 3\end{array}$ \\
\hline 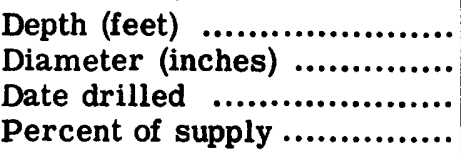 & $\begin{array}{r}946 \\
14 \\
1950 \\
--\end{array}$ & $\begin{array}{r}635 \\
14 \\
1948 \\
--\end{array}$ & $\begin{array}{r}529 \\
14 \\
1948 \\
--\end{array}$ & $\begin{array}{r}698 \\
14 \\
1948 \\
--\end{array}$ \\
\hline
\end{tabular}

ANALYSES

(Analyses, in parts per million, by Pomeroy \& Associates, Pasadena, California)

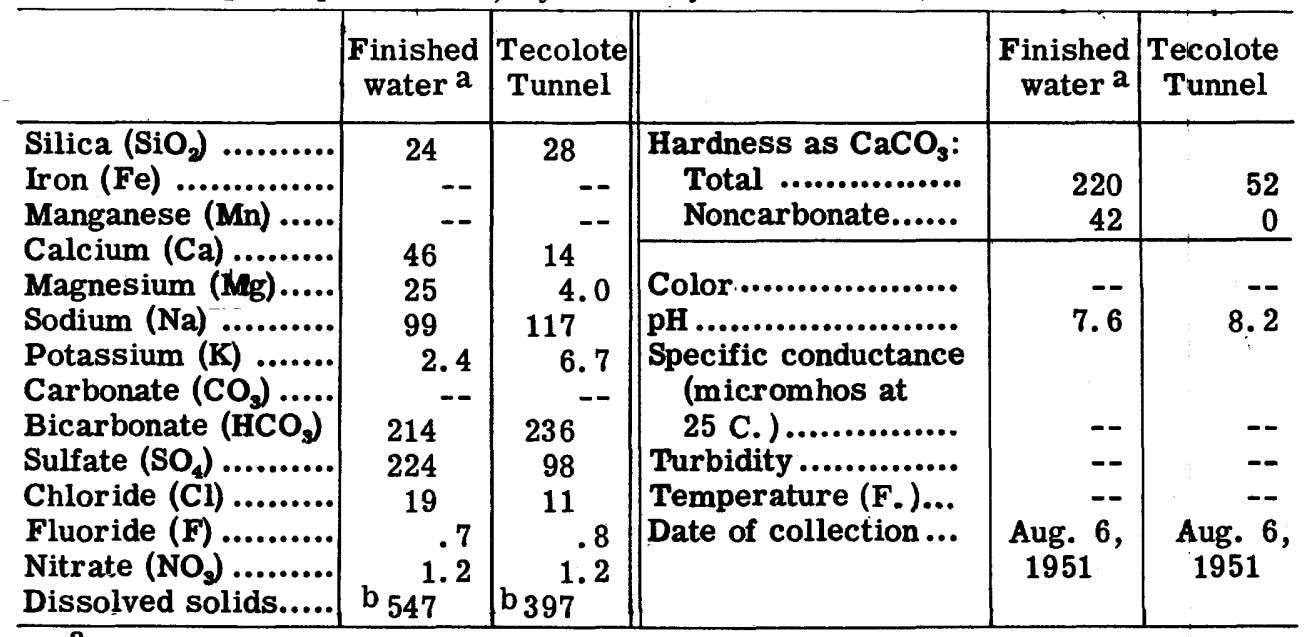

${ }^{\mathrm{a}}$ Sheffield Reservoir.

b Sum of determined constituents. 


\section{CALIFORNIA}

\section{SANTA CRUZ}

(Population, 21, 970)

Ownership: Municipal; supplies also about 11,000 people outside the city limits. Total population supplied, about 33,000 .

Source: Coastal streams: Laguna Creek, Majors Creek, and Liddell Creek (57 percent of supply); San Lorenzo River (39 percent of supply); 3 wells each 100 ft deep (4 percent of supply).

Treatment: San Lorenzo River: prechlorination, activated carbon, pressure filtration, and postchlorination. Coastal streams: chlorination. Wells: chlorination, and addition of Calgon for stabilization.

Rated capacity of treatment plant: 6, 750, 000 gpd.

Raw-water storage: None.

Finished-water storage: Reservoirs and tanks, 45, 800, 000 gal.

Water from the coastal streams flows by gravity to Santa Cruz. San Lorenzo River water is first treated, then pumped to the system.

\section{ANALYSES}

(Analyses, in parts per million, by California State Dept. of Public Health)

\begin{tabular}{|c|c|c|c|}
\hline & $\begin{array}{c}\text { San Lorenzo } \\
\text { River } \\
\text { (raw water) }\end{array}$ & $\begin{array}{c}\text { San Lorenzo } \\
\text { River } \\
\text { (finished water) }\end{array}$ & $\begin{array}{l}\text { Bay Street } \\
\text { Reservoir a }\end{array}$ \\
\hline Silica $\left(\mathrm{SiO}_{2}\right)$........................ & -- & -- & -- \\
\hline Iron $(\mathrm{Fe})$ & .1 & -- & -- \\
\hline Manganese (Mn) ..................... & .0 & -- & -- \\
\hline Calcium (Ca) $\ldots . . . \ldots \ldots \ldots \ldots \ldots \ldots . . . . . . . .$. & 33 & 37 & 55 \\
\hline Magnesium (Mg)..................... & 11 & 6.8 & 5.1 \\
\hline Sodium (Na)............................ & 22 & 20 & 12 \\
\hline Fotassium (K) $\ldots . . \ldots \ldots \ldots \ldots \ldots \ldots$ & -- & -- & - \\
\hline Carbonate $\left(\mathrm{CO}_{3}\right) \ldots \ldots \ldots \ldots \ldots \ldots \ldots$ & 0 & 0 & $\mathbf{0}$ \\
\hline Bicarbonate $\left(\mathrm{HCO}_{3}\right) \ldots \ldots \ldots \ldots \ldots$ & 110 & 126 & 177 \\
\hline 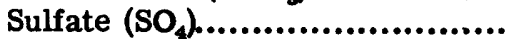 & 56 & 26 & 24 \\
\hline Chloride (Cl) ......................... & 20 & 24 & 12 \\
\hline Fluoride $(F) \ldots \ldots \ldots \ldots \ldots \ldots \ldots \ldots$ & .0 & .2 & .0 \\
\hline Nitrate $\left(\mathrm{NO}_{3}\right) \ldots \ldots \ldots \ldots \ldots \ldots \ldots \ldots$ & 1.5 & & .9 \\
\hline Dissolved solids .................... & $\mathrm{b}_{198}$ & 202 & 220 \\
\hline Hardness as $\mathrm{CaCO}_{3}$ : & & & \\
\hline Total $\ldots \ldots \ldots \ldots \ldots \ldots \ldots \ldots \ldots$ & 128 & 123 & 160 \\
\hline Noncarbonate ...................... & 37 & 20 & 15 \\
\hline Color. & -- & -- & -- \\
\hline pH.......... & 7.6 & 7.9 & 7.6 \\
\hline Specific conductance & & & \\
\hline (micromhos at 25 C. )... & -- & 347 & 370 \\
\hline Turbidity ........... & -- & $<1.0$ & $<1.0$ \\
\hline Temperature (F.) & & & \\
\hline Date of collection $. . . . . . . \ldots \ldots \ldots \ldots . .$. & Jan. 30, 1951 & Oct. 16,1951 & Oct. 16,1951 \\
\hline
\end{tabular}

${ }^{a}$ Coastal streams (finished water).

$\mathrm{b}_{\text {Sum of determined constituents. }}$ 


\section{CALIFORNIA}

SANTA MONICA

(Population, 71, 595)

Ownership: Municipal.

Source: Colorado River distributed by the Metropolitan Water District of Southern California, 91 percent of supply (See Los Angeles ); 8 wells ranging in depth from 250 to $468 \mathrm{ft}, 9$ percent of supply. The reported yield of the wells is from 500 to 1, $750 \mathrm{gpm}$. Emergency supply from Southern Californił Water Co.

Treatment: Colorado River water is softened and filtered by the Metropolitan Water District of Southern California. The well water is not treated.

Raw-water storage: --

Finished-water storage: 20,000, 000 gal.

\section{ANALYSES}

(Analyses, in parts per million, by California State Dept. of Public Health)

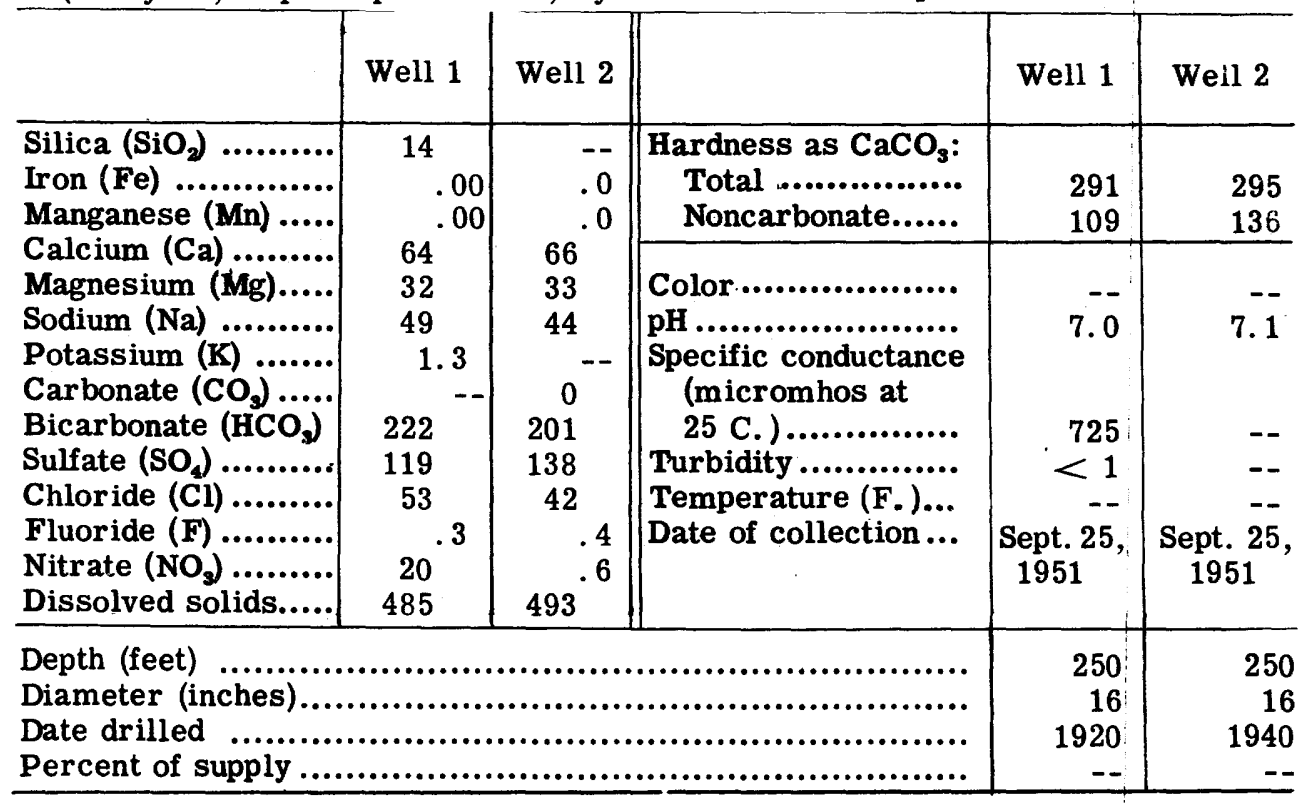




\section{SANTA ROSA}

(Population, 17, 902)

Ownership: Municipal; supplies also about 12,000 people outside the city limits. Total population supplied, about 30,000 .

Source: 7 wells (2 to 6, Peters Springs well, and Ralphine well) 900, 300, 1,000, 290, 915, 139, and $846 \mathrm{ft}$ deep, 67 percent of supply; Santa Rosa Creek and MacRae Springs, 33 percent of supply. MacRae Springs furnishes less than 1 percent of supply. The yield of the wells is reported to be $380,325,950$, 250, 1, 500, 575, and $450 \mathrm{gpm}$. Water from Santa Rosa Creek and MacRae Springs is stored in Lake Ralphine. Water from Peters Springs and Ralphine wells is pumped into Lake Ralphine during the summer months.

Treatment: Wells 2 to 6: aeration and po.yphosphate (Calgon) for iron and manganese control, chlorination, and dechlorination by sulfur dioxide. Lake Ralphine: chlorination after long storage.

Rated capacity of treatment plant: 6, 500,000 gpd.

Raw-water storage: Lake Ralphine, 135,000,000 gal.

Finished-water storage: Reservoirs, 2,800,000 gal.

\section{ANALYSES}

(Analyses, in parts per million, by Brown and Caldwell, San Francisco)

\begin{tabular}{|c|c|c|c|c|c|}
\hline & Well $4^{a}$ & Well 6 & $\begin{array}{l}\text { Peters } \\
\text { Springs } \\
\text { Wella }\end{array}$ & $\begin{array}{c}\text { Lake } \\
\text { Ralphine }\end{array}$ & $\begin{array}{l}\text { Finished } \\
\text { water } \\
\text { (city tap) }\end{array}$ \\
\hline 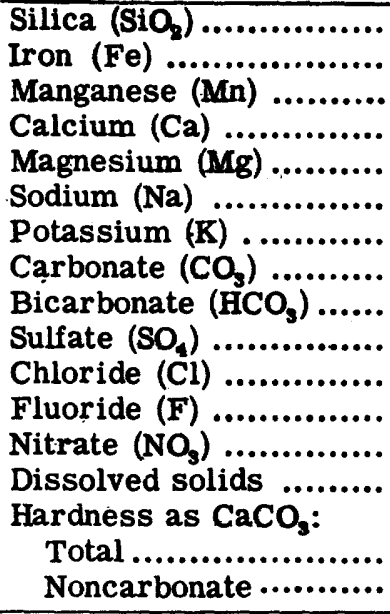 & $\begin{array}{c}93 \\
.33 \\
28 \\
.09 \\
18 \\
59 \\
0 \\
285 \\
27^{.9} \\
366^{-0} \\
144 \\
0\end{array}$ & $\begin{array}{c}89 \\
.27 \\
.07 \\
29 \\
18 \\
67 \\
0 \\
290 \\
9.9 \\
28 \\
7^{-0} \\
391 \\
147 \\
0\end{array}$ & $\begin{array}{l}95 \\
.33 \\
.10 \\
34 \\
21 \\
64 \\
0 \\
346 \\
2.9 \\
16 \\
{ }^{-0} \\
405 \\
171 \\
0\end{array}$ & 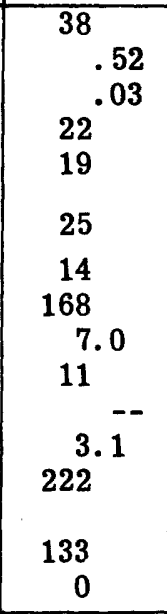 & $\begin{array}{c}84 \\
.27 \\
.18 \\
30 \\
18 \\
58 \\
0 \\
280 \\
8.8 \\
25 \\
1.5 \\
364 \\
149 \\
0 \\
\end{array}$ \\
\hline 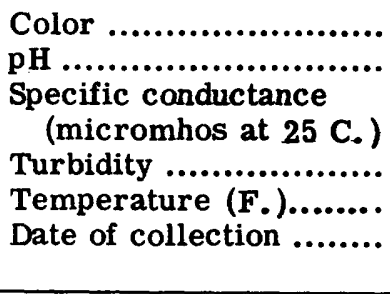 & 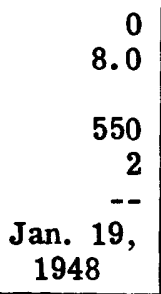 & $\begin{array}{r}7.4 \\
479 \\
-- \\
-- \\
\text { Aug. } 31, \\
1950\end{array}$ & $\begin{array}{r}0 \\
8.1 \\
610 \\
0 \\
-- \\
\text { Jan. } 22, \\
1948\end{array}$ & $\begin{array}{r}5 \\
8.4 \\
334 \\
7 \\
\text { Feb. } 10, \\
1949\end{array}$ & $\begin{array}{r}7.5 \\
486 \\
-- \\
\text { Sept. } 19, \\
1950\end{array}$ \\
\hline $\begin{array}{l}\text { Depth (feet) } \ldots . . . . . . . . . . . \\
\text { Diameter (inches)......... } \\
\text { Date drilled ............... } \\
\text { Percent of supply ........ }\end{array}$ & $\begin{array}{r}1,000 \\
16-10 \\
1940 \\
--\end{array}$ & $\begin{array}{r}915 \\
16 \\
1950 \\
--\end{array}$ & $\begin{array}{r}139 \\
8 \\
1923 \\
--\end{array}$ & & \\
\hline
\end{tabular}




\section{CALIFORNIA}

\section{SOUTH GATE}

(Population, 51, 116)

Ownership: Municipal.

Source: 17 wells ranging in depth from 551 to $1,600 \mathrm{ft}$. The reported yield of the individual wells is from 400 to $1,900 \mathrm{gpm}$. There are emergency crossconnections with the Huntington Park and Walnut Park systems.

Treatment: None.

Storage: $1,300,000$ gal.

\section{ANALYSES}

(Analyses, in parts per million, by Smith Emery Co., Los Angeles, Calif.)

\begin{tabular}{|c|c|c|c|c|c|}
\hline & Well 5 & Well 15 & Well 17 & Well 18 & Well 20 \\
\hline 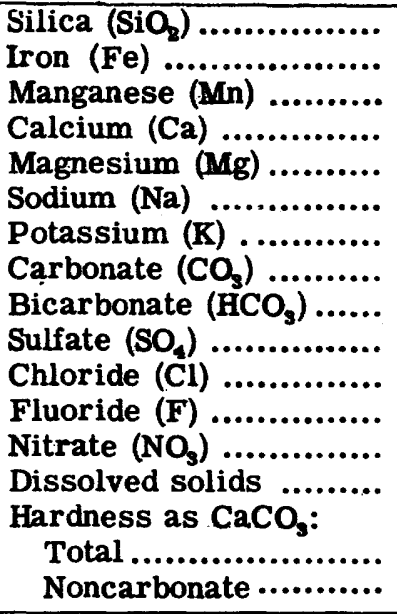 & $\begin{array}{c}16 \\
55 \\
8- \\
8.9 \\
47^{--} \\
0 \\
213 \\
59 \\
28 \\
-- \\
a_{319}^{--} \\
174 \\
0\end{array}$ & $\begin{array}{r}14 \\
-- \\
-- \\
68 \\
18 \\
46 \\
-- \\
0 \\
238 \\
103 \\
32 \\
-- \\
-- \\
398 \\
246 \\
48\end{array}$ & $\begin{array}{r}13 \\
-- \\
-- \\
60 \\
18 \\
46 \\
-- \\
0 \\
244 \\
64 \\
41 \\
-- \\
-- \\
a_{362} \\
225 \\
24\end{array}$ & $\begin{array}{r}12 \\
-- \\
-- \\
63 \\
12 \\
29 \\
-- \\
0 \\
238 \\
37 \\
26 \\
-- \\
-- \\
296 \\
208 \\
12\end{array}$ & $\begin{array}{r}14 \\
-- \\
-- \\
62 \\
14 \\
34 \\
-- \\
0 \\
219 \\
73 \\
23 \\
-- \\
-- \\
328 \\
214 \\
32 \\
\end{array}$ \\
\hline 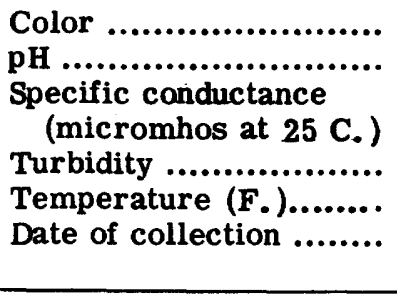 & $\begin{array}{r}7.4 \\
500 \\
-- \\
-- \\
\text { Nov. } 2, \\
1950\end{array}$ & $\begin{array}{r}7 . \overline{6} \\
600 \\
-- \\
-\overline{-} \\
\text { Nov. } 2 \text {, } \\
1950\end{array}$ & $\begin{array}{r}7.8 \\
570 \\
-- \\
-\overline{-} \\
\text { Nov. } 2, \\
1950\end{array}$ & $\begin{array}{r}7 . \overline{5} \\
450 \\
-- \\
-\overline{-} \\
\text { Nov. } 2, \\
1950\end{array}$ & $\begin{array}{r}7.8 \\
520 \\
-- \\
-- \\
\text { Nov. } 2 \text {, } \\
1950\end{array}$ \\
\hline $\begin{array}{l}\text { Depth (feet) } \ldots . \ldots \ldots \ldots \ldots . . \\
\text { Diameter (inches)........ } \\
\text { Date drilled .............. } \\
\text { Percent of supply ........ }\end{array}$ & $\begin{array}{r}1000 \\
12 \\
1923 \\
--\end{array}$ & $\begin{array}{r}700 \\
12 \\
-- \\
--\end{array}$ & $\begin{array}{r}551 \\
16 \\
1931 \\
--\end{array}$ & $\begin{array}{r}792 \\
18 \\
1945 \\
--\end{array}$ & $\begin{array}{r}1,400 \\
18 \\
1949 \\
--\end{array}$ \\
\hline
\end{tabular}

${ }^{\mathrm{a}}$ Sum of determined constituents. 


\section{CALIFORNIA}

\section{STOCKTON}

(Population, 70, 853)

Ownership: California Water Service Co. ; supplies also about 29, 000 people outside the city limits. Total population supplied, about 100, 000 .

Source: 37 wells ranging in depth from 198 to $1,050 \mathrm{ft}$.

Treatment: 7 wells at Station 1: aeration, chlorination, and Calgon. Water from all but 5 wells is chlorinated.

Rated capacity of treatment plant: --

Raw-water storage: None.

Finished-water storage: Reservoirs and tanks, 3, 000, 000 gal.

\section{ANALYSES}

(Analyses, in parts per million, by California Water Service Co., San Jose, Calif.)

\begin{tabular}{|c|c|c|c|c|c|}
\hline & $\begin{array}{l}\text { Well } \\
1-09\end{array}$ & $\begin{array}{l}\text { Well } \\
18-01\end{array}$ & $\begin{array}{l}\text { Well } \\
22-01\end{array}$ & $\begin{array}{c}\text { Range of } \\
\text { Con- } \\
\text { stituents }\end{array}$ & Mean $^{\mathrm{a}}$ \\
\hline 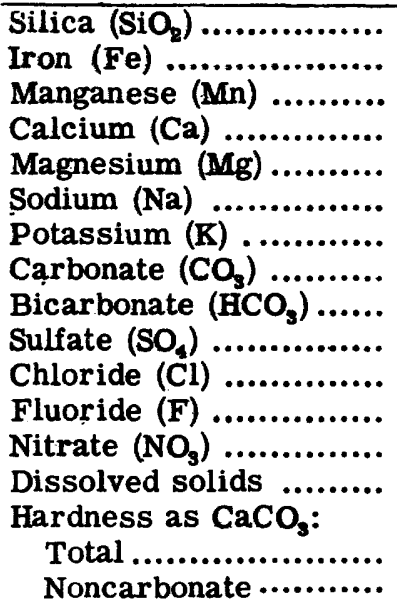 & 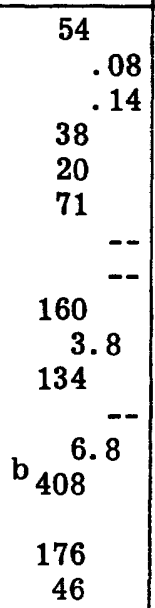 & $\begin{array}{c}43 \\
.04 \\
10 \\
4.08 \\
31.9 \\
4^{-0} \\
118 \\
3.4 \\
6 \\
b_{158} \\
.-\end{array}$ & 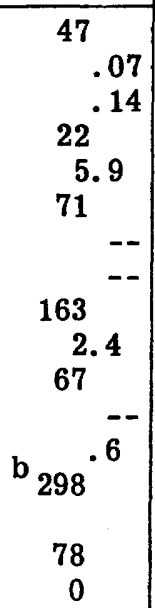 & 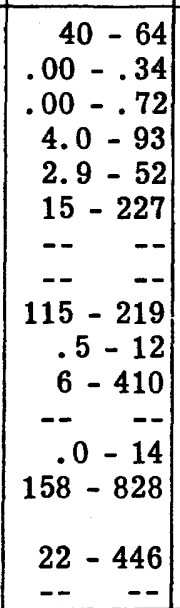 & $\begin{array}{c}49 \\
.08 \\
20 \\
10 \\
65 \\
\\
164 \\
4 .- \\
4.0 \\
63 \\
2.5^{--} \\
296 \\
91 \\
0\end{array}$ \\
\hline 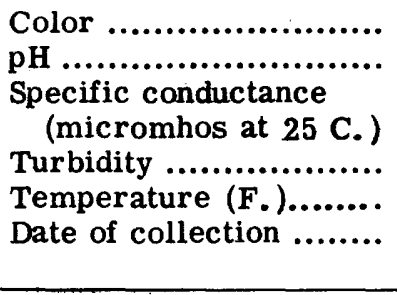 & $\begin{array}{r}7.8 \\
715 \\
-- \\
-- \\
\text { Apr. } 19 \\
1951 \\
\end{array}$ & $\begin{array}{r}211 \\
-- \\
-- \\
\text { July } 21, \\
1951\end{array}$ & $\begin{array}{r}8.0 \\
475 \\
-- \\
-- \\
\text { July } 21, \\
1951 \\
\end{array}$ & $\left|\begin{array}{rr}-- & -- \\
7.5 & -8.6 \\
211- & 1595 \\
-- & -- \\
-- & --\end{array}\right|$ & $\begin{array}{r}-- \\
8.0 \\
471 \\
-- \\
--\end{array}$ \\
\hline $\begin{array}{l}\text { Depth (feet) } \ldots . \ldots . \ldots . . . . \\
\text { Diameter (inches)........ } \\
\text { Date drilled .............. } \\
\text { Percent of supply ........ }\end{array}$ & $\begin{array}{l}-- \\
30 \\
-- \\
--\end{array}$ & $\begin{array}{r}408 \\
30-16 \\
1947 \\
--\end{array}$ & $\begin{array}{r}420 \\
30-16 \\
1949\end{array}$ & & \\
\hline
\end{tabular}

${ }^{a}$ Based on 37 analyses (1 analysis from each well) 1951.

b Sum of determined constituents. 


\section{CALIFORNIA}

\section{TORRANCE}

(Population, 22, 241)

Ownership: Municipal; supplies also about 4,200 people outside the city limits. Total population supplied, about 26,400 .

Source: 3 wells, 570, 540, and $492 \mathrm{ft}$ deep (60 percent of supply); Colorado River distributed by the Metropolitan Water District of Southern California, 40 percent of supply. (See Los Angeles.) The yield of the wells is reported to be $1,350,1,350$, and $950 \mathrm{gpm}$.

Treatment: Well water is chlorinated. Colorado River water is softened and filtered by the Metropolitan Water District of Southern California.

Storage: 2, 320, 000 gal.

\section{ANALYSES}

(Analyses, in parts per million, by Montgomery \& Pomeroy, Los Angeles, Calif.)

\begin{tabular}{|c|c|c|c|}
\hline & Well 1 & Well 2 & $\begin{array}{l}\text { Torrance } \\
\text { District } \\
\text { Well 1 }\end{array}$ \\
\hline 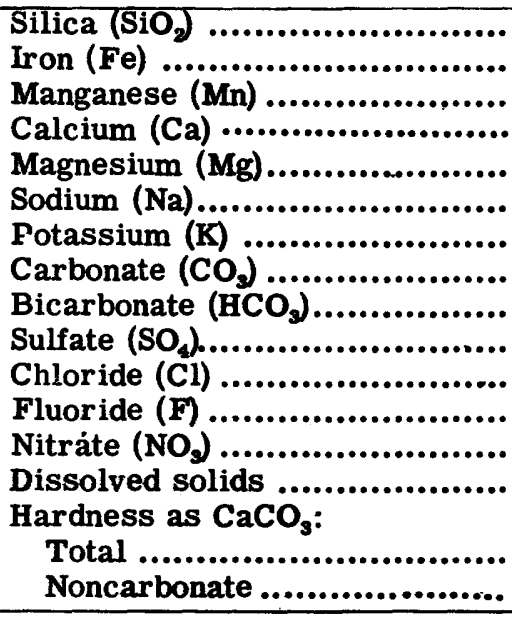 & $\begin{array}{c}.30 \\
36 \\
15 \\
73 \\
7.1 \\
0 \\
267 \\
68^{--} \\
a_{331} \\
.0 \\
150 \\
0 \\
\end{array}$ & $\begin{array}{c}.25 \\
33^{--} \\
14 \\
75 \\
7.9 \\
2722^{--} \\
63^{-0} \\
a_{327} \\
143 \\
0 \\
\end{array}$ & $\begin{array}{c}24 \\
0 \\
0 \\
39 \\
14 \\
69 \\
6.3 \\
0 \\
242 \\
7.3 \\
78 \\
.0 \\
-- \\
{ }^{2} 357 \\
154 \\
0 \\
0\end{array}$ \\
\hline 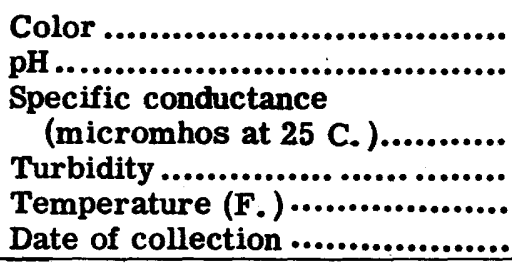 & $\begin{array}{r}-- \\
- \\
-- \\
0 \\
\text { Feb. } 15,1947\end{array}$ & $\begin{array}{r}-- \\
0 \\
\text { Feb. } 15,1947 \\
\end{array}$ & $\begin{array}{r}7 . \overline{7} \\
-- \\
0 \\
\text { Nov. } 12,1948 \\
\end{array}$ \\
\hline 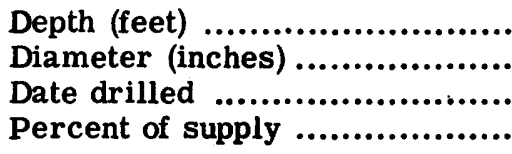 & $\begin{array}{r}570 \\
16 \\
1935 \\
--\end{array}$ & $\begin{array}{r}540 \\
16 \\
1935 \\
--\end{array}$ & $\begin{array}{r}492 \\
14 \\
1936 \\
--\end{array}$ \\
\hline
\end{tabular}

a Sum of determined constituents. 


\section{CALIFORNIA}

VALLEJO

(Population, 26, 038)

Ownership: Municipal; supplies also about 44, 350 people outside the city limits. . Total population supplied, about 70,400 .

Source: 61 percent of the supply is obtained from East Bay Municipal Utility District (see Oakland). Surface waters impounded in reservoirs: Gordon Valley Creek (26 percent of supply), Green Valley Creek (13 percent of supply).

Treatment: Water from East Bay Municipal Utility District treated by the District (see Oakland). Local supply: prechlorination, coagulation with alum, sedimentation, activated carbon, postchlorination, and lime for $\mathrm{pH}$ control.

Rated capacity of treatment plant: 5, 000,000 gpd.

Raw-water storage: Reservoirs, 6, 053, 600, 000 gal.

Finished-water storage: 49, 000, 000 gal.

\section{ANALYSES}

(Analyses, in parts per million, by City of Vallejo, Calif.)

\begin{tabular}{|c|c|c|c|c|c|}
\hline . & $\begin{array}{l}\text { Green } \\
\text { Valley } \\
\text { Creek }\end{array}$ & $\begin{array}{l}\text { Gordon } \\
\text { Valley } \\
\text { Creek }^{a}\end{array}$ & & $\begin{array}{l}\text { Green } \\
\text { Valley } \\
\text { Creek }\end{array}$ & $\begin{array}{l}\text { Gordon } \\
\text { Valley } \\
\text { Creek }^{a}\end{array}$ \\
\hline $\begin{array}{l}\text { Silica }\left(\mathrm{SiO}_{2}\right) \ldots \ldots \ldots \ldots . . \\
\text { Iron }(\mathrm{Fe}) \ldots \ldots \ldots \ldots \\
\text { Manganese }(\mathbf{M n}) \ldots \ldots .\end{array}$ & $\begin{array}{r}28 \\
.0 \\
.0\end{array}$ & $\begin{array}{l}5.0 \\
.0 \\
.0\end{array}$ & $\begin{array}{r}\text { Hardness as } \mathrm{CaCO}_{3} \text { : } \\
\text { Total ................... } \\
\text { Noncarbonate...... }\end{array}$ & $\begin{array}{r}29 \\
0 \\
\end{array}$ & $\begin{array}{r}170 \\
22 \\
\end{array}$ \\
\hline $\begin{array}{l}\text { Calcium (Ca) .......... } \\
\text { Magnesium ( } \mathrm{Mg}) \ldots . .\end{array}$ & $\begin{array}{l}6.0 \\
3.0\end{array}$ & & Color....... & 10 & 5 \\
\hline $\begin{array}{l}\text { Sodium (Na) ... } \\
\text { Potassium (K) }\end{array}$ & 10 & 27 & $\begin{array}{l}\text { pH .......................... } \\
\text { Specific conductance }\end{array}$ & 7.7 & 7.9 \\
\hline $\begin{array}{l}\text { Carbonate }\left(\mathrm{CO}_{3}\right) \ldots . . . . \\
\text { Bicarbonate }\left(\mathrm{HCO}_{3}\right)\end{array}$ & $\begin{array}{r}0 \\
37\end{array}$ & $\begin{array}{r}0 \\
183\end{array}$ & $\begin{array}{l}\text { (micromhos at } \\
25 \text { C. ) }\end{array}$ & -- & -- \\
\hline $\begin{array}{l}\text { Sulfate }\left(\mathrm{SO}_{4}\right) \ldots \ldots \ldots \ldots \\
\text { Chloride }(\mathrm{Cl}) \ldots \ldots . . . .\end{array}$ & $\begin{array}{r}10 \\
8\end{array}$ & 65 & $\begin{array}{l}\text { Turbidity ................ } \\
\text { Temperature (F.) }\end{array}$ & 10 & 25 \\
\hline Fluoride (F) ................ & ${ }^{8} .0$ & ${ }^{11} .0$ & Date of collection.... & June & June \\
\hline $\begin{array}{l}\text { Nitrate }\left(\mathrm{NO} \mathrm{O}_{3}\right) . . . . . . . . \\
\text { Dissolved solids...... }\end{array}$ & $90^{1.0}$ & $263^{.0}$ & & 1950 & 1950 \\
\hline
\end{tabular}

Regular determinations at treatment plant, 1950

\begin{tabular}{l|c|c|c|c|c|c|c|c|c|c|c|c}
\hline & \multicolumn{2}{|c|}{$\begin{array}{c}\text { Alkalinity } \\
\text { as CaCO } \\
\text { (ppm) }\end{array}$} & \multicolumn{3}{c|}{ pH } & \multicolumn{3}{c|}{$\begin{array}{c}\text { Hardness } \\
\text { as CaCO } \\
\text { (ppm) }\end{array}$} & \multicolumn{3}{c}{ Turbidity } \\
\cline { 2 - 12 } & Av & Max & Min & Av & Max & Min & Av & Max & Min & Av & Max & Min \\
\hline Raw water.......... & -- & -- & -- & 7.0 & 8.2 & 5.6 & 80 & 110 & 60 & 20 & 400 & 10 \\
Finished water... & -- & -- & -- & 7.4 & 7.4 & 7.4 & 80 & 110 & 60 & 2 & 5 & 0 \\
\hline
\end{tabular}

${ }^{a_{\text {Raw water. }}}$ 


\section{CALIFORNIA}

\section{WHITTIER}

(Population, 23, 820)

Ownership: Municipal.

Source: 6 wells $(1,5,7$ to 9 , and 11$), 434,680,1,000,302,664$, and $837 \mathrm{ft}$ deep. The yield of the wells is reported to be $800,1,800,2,200,4,300,3,750$, and $4,200 \mathrm{gpm}$.

Treatment: None.

Storage: Reservoirs, 18, 400, 000 gal.

\section{ANALYSES}

(Analyses, in parts per million, by Truesdail Laboratories Inc., Los Angeles)

\begin{tabular}{|c|c|c|c|c|c|}
\hline & Well 5 & Well 7 & Well 8 & Well 9 & Well 11 \\
\hline 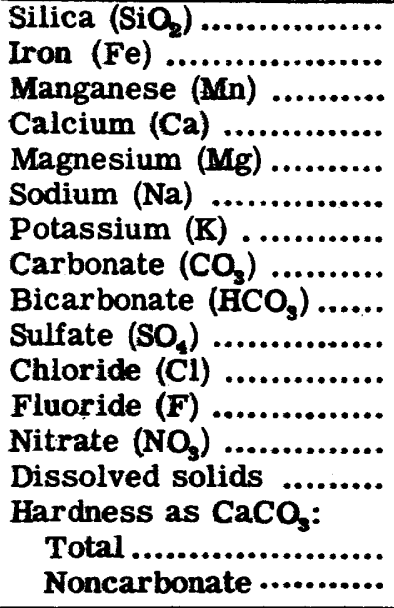 & $\begin{array}{c}25 \\
-- \\
83 \\
16 \\
34 \\
-- \\
0 \\
257 \\
101 \\
18 \\
-- \\
1.4 \\
405 \\
275 \\
62\end{array}$ & $\begin{array}{c}14 \\
61 \\
13 \\
20 \\
-- \\
0 \\
219 \\
40 \\
17 \\
4 .- \\
45 \\
277^{--} \\
205 \\
26\end{array}$ & $\begin{array}{c}18 \\
63 \\
63 \\
9.5 \\
13 \\
-- \\
0 \\
206 \\
24 \\
18 \\
-- \\
8.2 \\
255 \\
196 \\
27\end{array}$ & $\begin{array}{c}16 \\
44^{--} \\
6.0 \\
12 \\
0^{--} \\
159 \\
1.9 \\
20^{--} \\
a_{178} \\
134 \\
4\end{array}$ & $\begin{array}{l}20 \\
33 \\
3.8 \\
22^{--} \\
0 \\
144^{--} \\
15^{.0} \\
2.2 \\
167^{--} \\
97 \\
0\end{array}$ \\
\hline 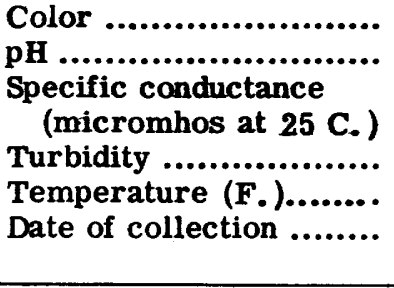 & $\begin{array}{r}7.2 \\
672 \\
-- \\
-- \\
\text { May } 21, \\
1951 \\
\end{array}$ & $\begin{array}{r}7 . \overline{2} \\
453 \\
-- \\
-\overline{-} \\
\text { Jan. } 18 \\
1951\end{array}$ & $\begin{array}{r}8.0 \\
406 \\
-- \\
-\overline{18} \\
\text { Jan. } 18 \\
1951\end{array}$ & $\begin{array}{r}7 . \overline{9} \\
291 \\
-- \\
-\overline{18} \\
\text { Jan. } 18, \\
1951\end{array}$ & $\begin{array}{r}8.1 \\
297 \\
-- \\
\text { Jan. } 18, \\
1951\end{array}$ \\
\hline $\begin{array}{l}\text { Depth (feet) } \ldots . . . . . . . . . . . \\
\text { Diameter (inches)........ } \\
\text { Date drilled ............... } \\
\text { Percent of supply ......... }\end{array}$ & $\begin{array}{r}680 \\
26-18 \\
1922 \\
--\end{array}$ & $\begin{array}{r}1,000 \\
18-14 \\
1930 \\
--\end{array}$ & $\begin{array}{r}302 \\
24 \\
1931 \\
--\end{array}$ & $\begin{array}{r}664 \\
24 \\
1933 \\
--\end{array}$ & $\begin{array}{r}837 \\
20 \\
1948 \\
--\end{array}$ \\
\hline
\end{tabular}

a Sum of determined constituents. 


\section{OREGON}

\section{OREGON}

\section{ALBANY}

(Population, 10,115)

Ownership: Mountain States Power Co. ; supplies also about 330 people outside the city limits. Total population supplied, about 10,450.

Source: South Santiam River (through power canal).

Treatment: Coagulation with alum and lime, rapid sand filtration, and chlorination. Rated capacity of treatment plant: $3,000,000$ gpd.

Raw-water storage: None.

Finished-water storage: 890,000 gal.

\section{ANALYSIS}

(Analysis, in parts per million, by Elgin Water Softener Corp., Elgin, Ill.)

\begin{tabular}{|c|c|c|c|}
\hline & $\begin{array}{l}\text { Finished } \\
\text { water }\end{array}$ & & $\begin{array}{c}\text { Finished } \\
\text { water }\end{array}$ \\
\hline $\begin{array}{l}\text { Silica }\left(\mathrm{SiO}_{2}\right) \ldots \ldots \ldots \ldots \ldots . \\
\text { Iron }(\mathrm{Fe}) \quad \ldots \ldots \ldots \ldots \ldots \ldots \\
\text { Manganese }(\mathrm{Mn}) \ldots \ldots \ldots \ldots\end{array}$ & $\begin{array}{r}17 \\
-- \\
--\end{array}$ & $\begin{array}{l}\text { Hardness as } \mathrm{CaCO}_{3}: \\
\text { Total ....................... } \\
\text { Noncarbonate ........... }\end{array}$ & $\begin{array}{l}42 \\
15 \\
\end{array}$ \\
\hline 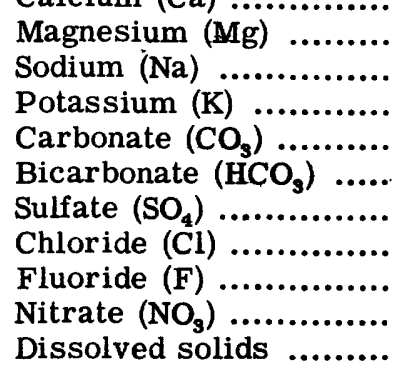 & $\begin{array}{l}1.2 \\
4.3 \\
-- \\
0 \\
33 \\
19 \\
3.6 \\
\mathrm{a} .2 \\
\mathrm{~b} .- \\
76\end{array}$ & 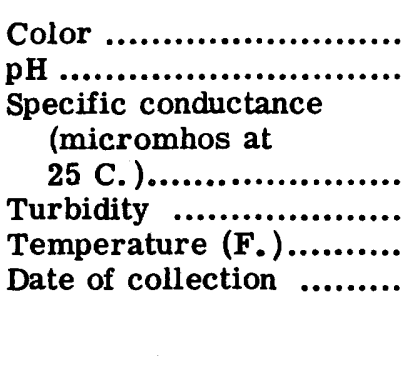 & Apr. 12 , \\
\hline
\end{tabular}

Regular determinations at treatment plant,

\begin{tabular}{l|c|c|c|c|c|c|c|c|c|c|c|c}
\hline & \multicolumn{3}{|c|}{$\begin{array}{c}\text { Alkalinity } \\
\text { as CaCO } \\
\text { (ppm) }\end{array}$} & \multicolumn{3}{c|}{ pH } & \multicolumn{3}{c|}{$\begin{array}{c}\text { Hardness } \\
\text { as CaCO } \\
\text { (ppm) }\end{array}$} & \multicolumn{3}{c}{ Turbidity } \\
\cline { 2 - 13 } & Av & Max & Min & Av & Max & Min & Av & Max & Min & Av & Max & Min \\
\hline Raw water......... & -- & -- & -- & -- & -- & -- & -- & -- & - & -- & -- & -- \\
Finished water... & 27 & -- & -- & 7.0 & -- & -- & 43 & -- & -- & -- & -- & -- \\
\hline
\end{tabular}

${ }^{\text {a}}$ Determination by Oregon State Board of Health, November 1950.

${ }^{b}$ Sum of determined constituents. 


\section{OREGON}

\section{ASTORIA}

(Population, 12, 331)

Ownership: Municipal; supplies also Tongue Point Naval Station and some other consumers outside the city limits. Total population supplied, about 14,800 .

Source: Bear Creek and tributaries impounded in 3 artificial lakes. Intake located about 11 miles east of Astoria.

Treatment: Chlorination and occasional use of copper sulfate for algae control.

Rated capacity of treatment plant: 5, 200,000 gpd.

Raw water storage: 200,000,000 gal.

Finished water storage: 24,000,000 gal.

The color and turbidity of the water are variable, being highest from September through November.

\section{ANALYSIS}

(Analysis, in parts per million, by 13th Naval Dist. Sanitation Engineering Lab.)

\begin{tabular}{|c|c|c|c|}
\hline & $\begin{array}{l}\text { Finished } \\
\text { water }\end{array}$ & & $\begin{array}{c}\text { Finished } \\
\text { water }\end{array}$ \\
\hline $\begin{array}{l}\text { Silica }\left(\mathrm{SiO}_{2}\right) \\
\text { Iron }(\mathrm{Fe}) \\
\text { Manganese }(\mathbf{M n})\end{array}$ & $0^{28} .18$ & 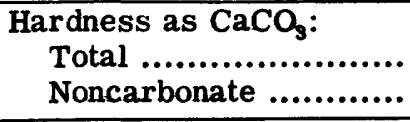 & $\begin{array}{r}27 \\
0 \\
\end{array}$ \\
\hline $\begin{array}{l}\text { Magnesium }(\mathrm{Mg}) \\
\text { Sodium }(\mathrm{Na}) \\
\text { Potassium }(\mathrm{K}) \\
\text { Carbonate }\left(\mathrm{CO}_{3}\right) \\
\text { Bicarbonate }\left(\mathrm{HCO}_{3}\right)\end{array}$ & $\begin{array}{l}2.8 \\
7.6 \\
0 \\
37 \\
3.2 \\
6.8 \\
\mathrm{a}^{.} \\
\quad-\end{array}$ & 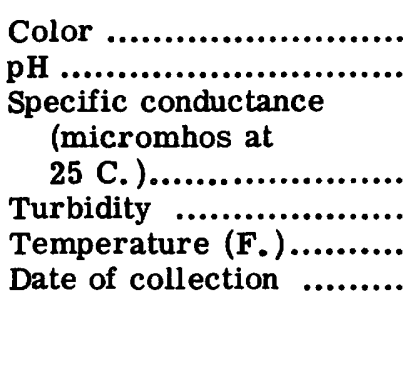 & $\begin{array}{r}10 \\
7.0 \\
-- \\
2 \\
\text { June } \\
1950\end{array}$ \\
\hline
\end{tabular}

Regular determinations at treatment plant, 1950

\begin{tabular}{l|c|c|c|c|c|c|c|c|c|c|c|c|c}
\hline & \multicolumn{3}{|c|}{$\begin{array}{c}\text { Alkalinity } \\
\text { as CaCO } \\
\text { (ppm) }\end{array}$} & \multicolumn{3}{c|}{ pH } & \multicolumn{3}{c|}{$\begin{array}{c}\text { Hardness } \\
\text { as CaCO } \\
\text { (ppm) }\end{array}$} & \multicolumn{3}{c}{$\begin{array}{c}\text { Tempera- } \\
\text { ture }\end{array}$} \\
\cline { 2 - 13 } & Av & Max & Min & Av & Max & Min & Av & Max & Min & Av & Max & Min \\
\hline Raw water......... & -- & -- & -- & 7.0 & 7.6 & 6.8 & -- & -- & -- & 50 & 67 & 37 \\
Finished water... & -- & -- & -- & 6.9 & 7.0 & 6.8 & -- & -- & -- & 49 & 62 & 35 \\
\hline
\end{tabular}

${ }^{\text {a }}$ rom other analyses. 


\section{OREGON}

\section{BEND}

(Population 11, 409)

Ownership: Municipal; supplies also about 300 people outside the city limits.

Total population supplied, about 11,700 .

Source: Tumalo Creek.

Treatment: Chlorination.

Raw water storage: None.

Finished water storage: 1,635,000 gal.

\section{ANALYSIS}

(Analysis, in parts per million, by U. S. Geological Survey)

\begin{tabular}{|c|c|c|c|}
\hline & $\begin{array}{c}\text { Finished } \\
\text { water }\end{array}$ & & $\begin{array}{c}\text { Finished } \\
\text { water }\end{array}$ \\
\hline $\begin{array}{l}\text { Silica }\left(\mathrm{SiO}_{2}\right) \ldots \ldots \ldots \ldots \ldots \ldots \\
\text { Iron }(\mathrm{Fe}) \quad \ldots \ldots \ldots \ldots \ldots \ldots \ldots \\
\text { Manganese }(\mathrm{Mn}) \ldots \ldots \ldots \ldots\end{array}$ & $\begin{array}{r}19 \\
.03 \\
--\end{array}$ & $\begin{array}{l}\text { Hardness as } \mathrm{CaCO}_{\mathbf{3}}: \\
\text { Total } \ldots \ldots \ldots \ldots \ldots \ldots \ldots \ldots \\
\text { Noncarbonate } \ldots \ldots \ldots \ldots \ldots\end{array}$ & $\begin{array}{r}11 \\
0\end{array}$ \\
\hline 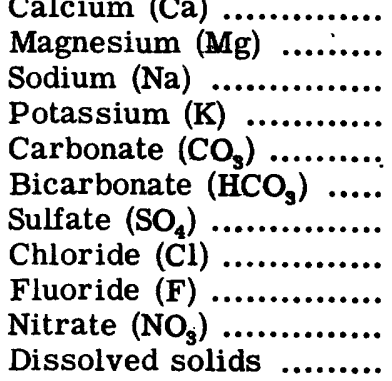 & $\begin{array}{r}3.5 \\
.6 \\
2.0 \\
.8 \\
0 \\
18 \\
.8 \\
.9 \\
.0 \\
.1\end{array}$ & 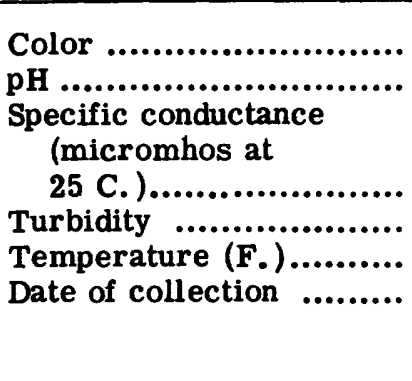 & $\begin{array}{r}7 \\
6.7 \\
\\
31 \\
- \\
46 \\
\text { June } 18, \\
1951\end{array}$ \\
\hline
\end{tabular}




\section{OREGON}

\section{COOS BAY}

(Population, 6, 223).

Ownership: Municipal; supplies also North Bend, Eastside, and about 1, 500 consumers outside the city limits. Total population supplied, about $15,200$.

Source: Pony Creek.

Treatment: Prechlorination, color removal by coagulation with alum and lime, rapid sand filtration, and aeration (spray).

Rated capacity of treatment plant: $4,000,000$ gpd.

Raw-water storage: 658,000,000 gal.

Finished-water storage: 5, 500,000 gal. Present construction program will provide an additional 4, 500,000 gal.

\section{ANALYSES}

(Analyses, in parts per million, by U. S. Geological Survey)

\begin{tabular}{|c|c|c|c|c|c|}
\hline & $\begin{array}{c}\text { Raw } \\
\text { water }^{\mathrm{a}}\end{array}$ & $\begin{array}{c}\text { Finished } \\
\text { water }\end{array}$ & & $\begin{array}{c}\text { Raw } \\
\text { watera }\end{array}$ & $\begin{array}{c}\text { Finished } \\
\text { water }\end{array}$ \\
\hline $\begin{array}{l}\text { Silica }\left(\mathrm{SiO}_{2}\right) \\
\text { Iron }(\mathrm{Fe}) \ldots \ldots \ldots \ldots \\
\text { Manganese }(\mathrm{Mn}) \ldots \ldots\end{array}$ & $\begin{array}{c}7.1 \\
.08 \\
--\end{array}$ & $\begin{array}{c}6.6 \\
.04 \\
--\end{array}$ & $\begin{array}{r}\text { Hardness as } \mathrm{CaCO}_{3} \text { : } \\
\text { Total ................. } \\
\text { Noncarbonate...... }\end{array}$ & $\begin{array}{l}9 \\
0 \\
\end{array}$ & $\begin{array}{l}37 \\
18 \\
\end{array}$ \\
\hline 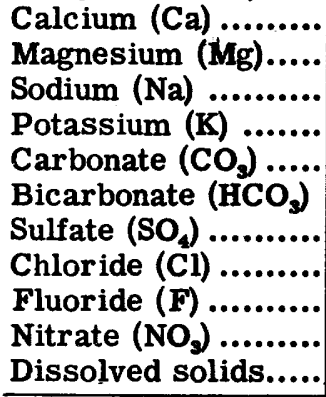 & $\begin{array}{c}1.8 \\
1.0 \\
5.9 \\
0 \\
0 \\
16 \\
2.1 \\
10^{-} \\
.2 \\
.4 \\
57^{4}\end{array}$ & $\begin{array}{l}12 \\
1.8 \\
8.3 \\
1.4 \\
0 \\
23 \\
13 \\
15 \\
.0 \\
.2\end{array}$ & 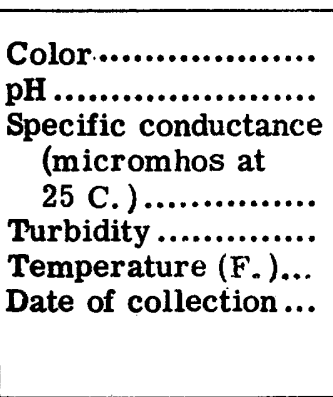 & $\begin{array}{r}-- \\
5 \\
58 \\
\text { May } 7 \\
1947\end{array}$ & $\begin{array}{r}116 \\
-- \\
66 \\
\text { June 15, } \\
1951\end{array}$ \\
\hline
\end{tabular}

Regular determinations at treatment plant, 1950

\begin{tabular}{|c|c|c|c|c|c|c|c|c|c|c|c|c|}
\hline & \multicolumn{3}{|c|}{$\begin{array}{c}\text { Alkalinity } \\
\text { as CaCO } \\
\text { (ppm) }\end{array}$} & \multicolumn{3}{|c|}{ pH } & \multicolumn{3}{|c|}{$\begin{array}{l}\text { Hardness } \\
\text { as CaCO } \\
\text { (ppm) }\end{array}$} & \multicolumn{3}{|c|}{$\begin{array}{l}\text { Tempera- } \\
\text { ture }\end{array}$} \\
\hline & $\mathrm{Av}$ & $\operatorname{Max}$ & Min & Av & $\operatorname{Max}$ & Min & Av & $\operatorname{Max}$ & Min & Av & $\operatorname{Max}$ & Min \\
\hline $\begin{array}{l}\text { Raw water......... } \\
\text { Finished water... }\end{array}$ & $\begin{array}{l}12 \\
18\end{array}$ & $\begin{array}{l}14 \\
24\end{array}$ & $\begin{array}{r}8 \\
16\end{array}$ & $\begin{array}{l}6.6 \\
8.4\end{array}$ & \begin{tabular}{|l|}
6.9 \\
9.0
\end{tabular} & $\begin{array}{l}5.2 \\
8.0\end{array}$ & $\begin{array}{r}8 \\
24\end{array}$ & $\begin{array}{l}10 \\
30\end{array}$ & $\begin{array}{r}8 \\
22\end{array}$ & $\begin{array}{l}54 \\
--\end{array}$ & $\begin{array}{l}68 \\
--\end{array}$ & $\begin{array}{l}36 \\
--\end{array}$ \\
\hline
\end{tabular}

a Analyzed by the Charlton Laboratories, Portland, Oreg. 


\section{OREGON}

\section{CORVALLIS}

(Population, 16, 207)

Ownership: Municipal; supplies also Philomath and about 800 consumers outside the city limits. Total population supplied, about 18,300.

Source: Rock Creek.

Treatment: Pressure filtration, chlorination and ammoniation.

Rated capacity of treatment plant: 5,000,000 gpd.

Raw-water storage: --

Finished-water storage: 7,000,000 gal.

A water treatment plant has been constructed to utilize water from the Willamette River although this source has not been used to date (June 1951). This plant has a rated capacity of 4,000,000 gpd. The treatment of the water will include coagulation, sedimentation, gravity filtration, and chlorination.

\section{ANALYSES}

(Analyses, in parts per million, by U. S. Geological Survey)

\begin{tabular}{|c|c|c|c|c|c|}
\hline & $\begin{array}{c}\text { Finished } \\
\text { water }\end{array}$ & $\underset{\text { waterb }}{\text { Raw }}$ & & $\begin{array}{c}\text { Finished } \\
\text { water }\end{array}$ & $\begin{array}{l}\text { Raw } \\
\text { water }\end{array}$ \\
\hline $\begin{array}{l}\text { Silica }\left(\mathrm{SiO}_{2}\right) \\
\text { Iron }(\mathrm{Fe}) \ldots \ldots \ldots \ldots \\
\text { Manganese }(\mathrm{Mn})\end{array}$ & $\begin{array}{r}24 \\
.02 \\
--\end{array}$ & 19 & $\begin{array}{r}\text { Hardness as } \mathrm{CaCO}_{3} \text { : } \\
\text { Total .................. } \\
\text { Noncarbonate...... }\end{array}$ & $\begin{array}{r}37 \\
0\end{array}$ & $\begin{array}{r}20 \\
0\end{array}$ \\
\hline $\begin{array}{l}\text { Calcium }(\mathrm{Ca}) . . . . . . \\
\text { Magnesium }(\mathrm{Mg}) . . .\end{array}$ & $\begin{array}{l}8.5 \\
3.9\end{array}$ & $\begin{array}{l}4.2 \\
2.4\end{array}$ & Color.................... & 5 & -- \\
\hline Sodium (Na) ........... & 5.1 & 5.8 & pH $\ldots \ldots \ldots \ldots \ldots \ldots \ldots$ & 7.5 & -- \\
\hline $\begin{array}{l}\text { Potassium }(\mathbf{K}) \\
\text { Carbonate }(\mathrm{CO}, \ldots . . .\end{array}$ & $\begin{array}{l}2.2 \\
0\end{array}$ & 0 & (micromhos at & & \\
\hline Bicarbonate (HCO, & 51 & 28 & 25 C. $). . . . . . . . . . . . .$. & 94 & 47.9 \\
\hline Sulfate $\left(\mathrm{SO}_{4}\right) \ldots . . . .$. & 1.8 & 4.9 & Turbidity & -- & -- \\
\hline Chloride (Cl). & 4.8 & 3.0 & Temperature (F.)... & 64 & -- \\
\hline Fluoride (F) ..... & .2 & -- & Date of collection... & June 14, & Nov. 27, \\
\hline $\begin{array}{l}\text { Nitrate }\left(\mathrm{NO}_{2}\right) . . . . . . . . \\
\text { Dissolved solids.... }\end{array}$ & $76^{.2}$ & $c_{54}^{.8}$ & & 1951 & 1950 \\
\hline
\end{tabular}

${ }^{\text {a }}$ Rock Creek.

$b_{\text {Willamette River. }}$

$\mathbf{c}_{\text {Sum of determined constituents. }}$ 


\section{OREGON}

\section{EUGENE}

(Population, 35, 879)

Ownership: Municipal; supplies also about 14,000 people outside the city limits. Total population supplied, approximately 50,000.

Source: McKenzie River. The intake is located near Hayden Bridge, 7 miles east of the city.

Treatment: Prechlorination, coagulation with alum and lime at times of high turbidity of water, and rapid sand filtration.

Rated capacity of treatment plant: 25,000,000 gpd.

Raw-water storage: None.

Finished-water storage: 5 reservoirs, 21, 100,000 gal; elevated tank, 100,000 gal.

\section{ANALYSIS}

(Analysis, in parts per million, by U. S. Geological Survey)

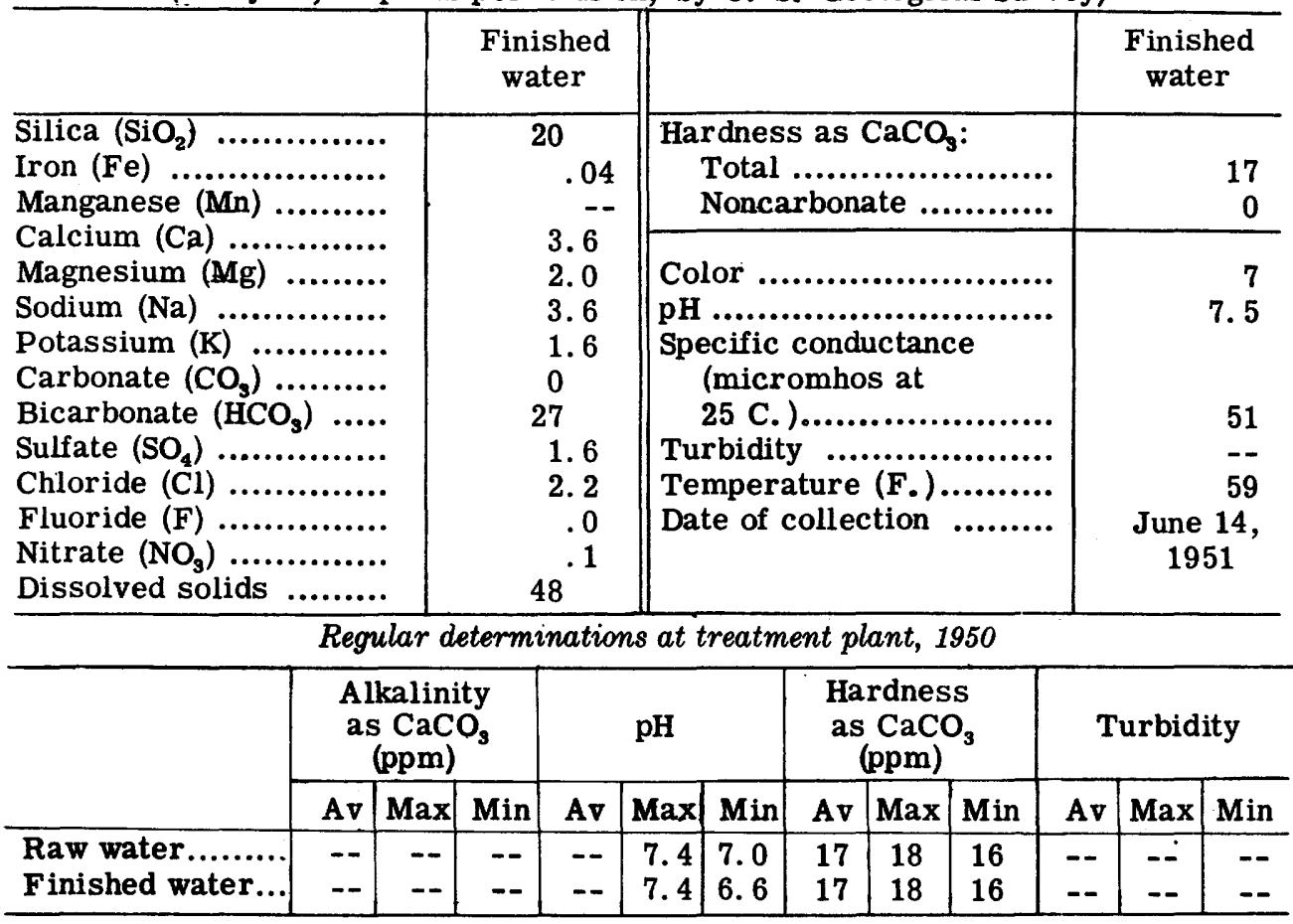




\section{KLAMATH FALLS}

(Population, 15, 875)

Ownership: Oregon Water Corporation (subsidiary of Boise Water Corporation,

Boise, Idaho); supplies suburban Altamont District and also consumers outside the corporate limits of the city. Total populaticn supplied, about 26,000.

Source: 4 flowing wells (3, 6, 7, and 8), 145, 147, 370, and $850 \mathrm{ft}$ deep.

Treatment: Chlorination.

Raw-water storage: None.

Finished-water storage: $3,700,000$ gal.

\section{ANALYSIS}

(Analysis, in parts per million, by the University of California, Berkeley, Calif.)

\begin{tabular}{|c|c|c|c|}
\hline & $\begin{array}{l}\text { Wells } \\
\text { (composite } \\
\text { sample) }\end{array}$ & & $\begin{array}{c}\text { Wells } \\
\text { (composite } \\
\text { sample) }\end{array}$ \\
\hline $\begin{array}{l}\text { Silica }\left(\mathrm{SiO}_{2}\right) \\
\text { Iron }(\mathrm{Fe}) \ldots \ldots \ldots \ldots \ldots \ldots \\
\text { Manganese }(\mathrm{Mn}) \ldots \ldots \ldots \ldots\end{array}$ & $0^{24} .15$ & $\begin{array}{l}\text { Hardness as CaCO } \\
\text { Total ....................... } \\
\text { Noncarbonate ........... }\end{array}$ & $\begin{array}{r}52 \\
0\end{array}$ \\
\hline 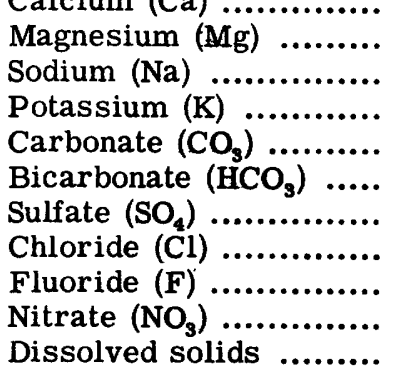 & $\begin{array}{c}6.3 \\
30 \\
108^{--} \\
1.3 \\
19^{-3} \\
141^{--}\end{array}$ & 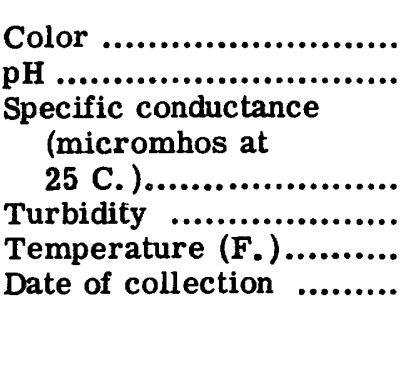 & $\begin{array}{r}212 \\
-- \\
67 \\
\text { Mar. } 22, \\
1949\end{array}$ \\
\hline
\end{tabular}




\section{OREGON}

\section{MEDFORD}

(Population, 17, 305)

Ownership: Municipal; supplies also Central Point, Eagle Point, and about 3,500 people outside the city limits. Total population supplied, about 23,100.

Source: Big Butte Spring.

Treatment: None.

Storage: $12,400,000$ gal.

\begin{tabular}{|c|c|c|c|}
\hline \multicolumn{4}{|c|}{ ANALYSIS } \\
\hline & $\begin{array}{l}\text { Big Butte } \\
\text { Springs }\end{array}$ & & $\begin{array}{l}\text { Big Butte } \\
\text { Springs }\end{array}$ \\
\hline $\begin{array}{l}\text { Silica }\left(\mathrm{SiO}_{2}\right) \ldots \ldots \ldots \ldots \ldots \ldots \\
\text { Iron }(\mathrm{Fe}) \quad \ldots \ldots \ldots \ldots \ldots \ldots \\
\text { Manganese }(\mathrm{Mn}) \ldots \ldots \ldots \ldots\end{array}$ & $\begin{array}{r}35 \\
.0\end{array}$ & $\begin{array}{l}\mathrm{Hardness} \text { as } \mathrm{CaCO}_{3}: \\
\quad \text { Total ...................... } \\
\text { Noncarbonate ........... }\end{array}$ & $\begin{array}{r}35 \\
0\end{array}$ \\
\hline $\begin{array}{l}\text { Calcium (Ca) ............... } \\
\text { Magnesium (Mg) }\end{array}$ & $\begin{array}{l}7.3 \\
4.0\end{array}$ & Color ........ & -- \\
\hline 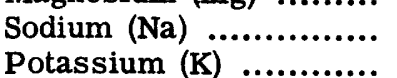 & 7.0 & 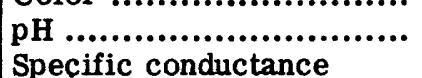 & 6.9 \\
\hline $\begin{array}{l}\text { Carbonate }\left(\mathrm{CO}_{3}\right) \ldots \ldots \ldots . . \\
\text { Bicarbonate }(\mathrm{HCO})\end{array}$ & $\begin{array}{r}0 \\
56\end{array}$ & (micromhos at & - \\
\hline Sulfate $\left(\mathrm{SO}_{4}\right) \ldots \ldots \ldots \ldots$ & 1.3 & 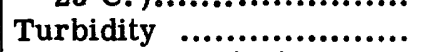 & -- \\
\hline 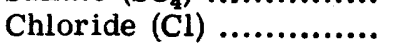 & 2.0 & Temperature (F.) & 42 \\
\hline Fluoride $(F)$.............. & .0 & Date of collection & Apr. 21, \\
\hline $\begin{array}{l}\text { Nitrate }\left(\mathrm{NO}_{3}\right) \ldots \ldots \ldots \ldots . . . . \\
\text { Dissolved solids .......... }\end{array}$ & $99^{.3}$ & & 1947 \\
\hline
\end{tabular}




\section{OREGON \\ PENDLETON \\ (Population 11, 774)}

Ownership: Municipal; supplies also about 200 people outside the city limits.

Total population supplied, about 12,000 .

Source: Springs, 80 percent of supply; 2 wells 774 and $761 \mathrm{ft}$ deep, 20 percent of supply. Emergency supply from privately owned well.

Treatment: Chlorination.

Raw-water storage: None.

Finished-water storage: $3,500,000 \mathrm{gal}$.

\section{ANALYSIS}

(Analysis, in parts per million, by Charlton Laboratories, Portland, Oreg.)

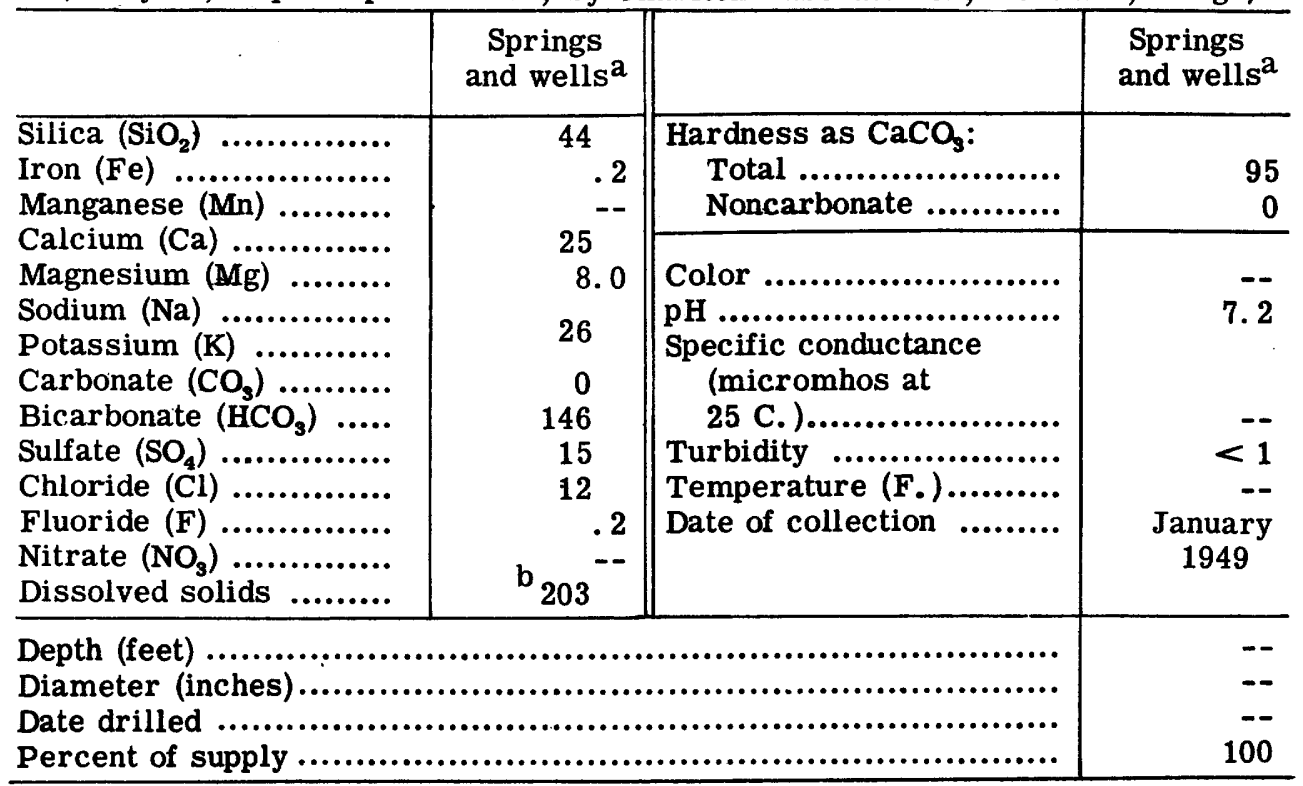

${ }^{\mathrm{a}}$ Composite sample.

$\mathrm{b}_{\text {Sum }}$ of determined constituents. 


\section{OREGON}

\section{PORTLAND}

(Population, 373, 628)

Ownership: Municipal; supplies also about 107,000 people outside the city limits, including 60 water districts, water companies, and towns in areas adjacent to Portland. Total population supplied, about 480,000 .

Source: Bull Run River impounded in Lake Ben Morrow Reservoir which extends $3 \frac{1}{2}$ miles along the river, and Bull Run Lake, the source of the main branch of the river close to the summit of the Cascades.

Treatment: Chlorination and ammoniation.

Rated capacity of treatment plant:

Raw-water storage: Storage reservoir on Bull Run River, 11, 000,000,000 gal; Bull Run Lake, 3,000,000,000 gal.

Finished-water storage: 6 reservoirs, $192,000,000$ gal.

The water from the river is diverted 5 miles downstream from the storage dam through 3 steel conduits to distribution reservoirs on Mount Tabor, a distance of 24 miles. Four distribution reservoirs are located on Mount Tabor and two in Washington Park. Distribution is mainly by gravity.

\section{ANALYSIS}

(Analysis, in parts per million, by Charlton Laboratories, Portland, Oreg.)

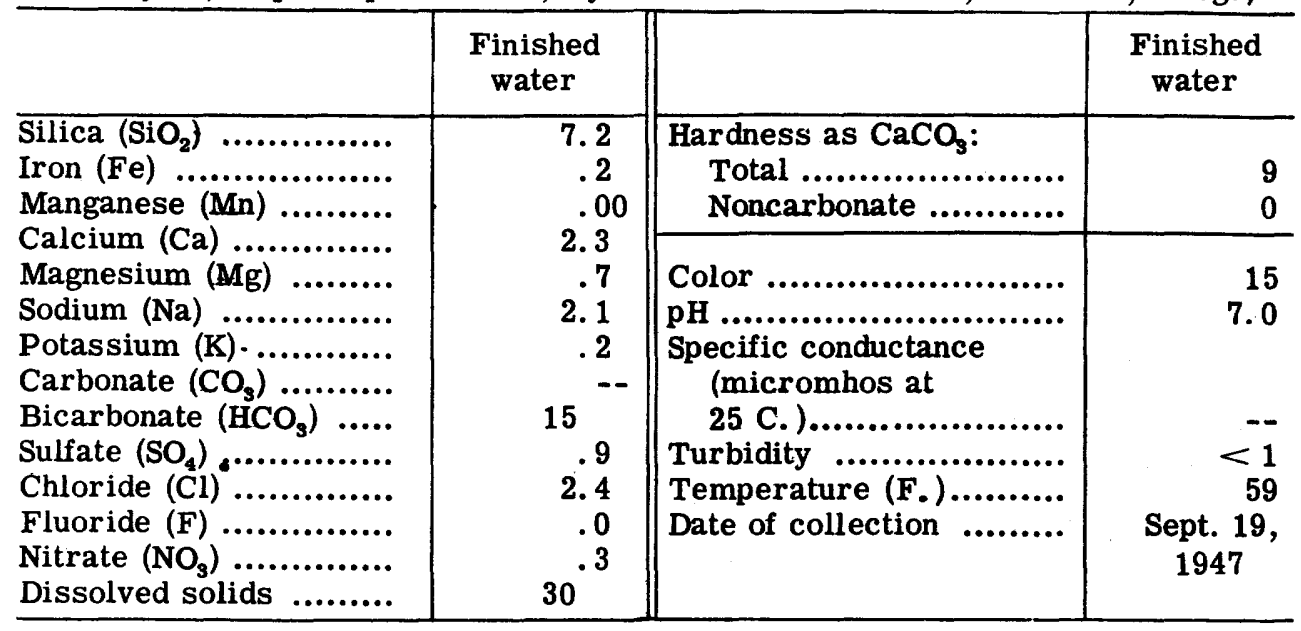




\section{OREGON}

SALEM

(Population, 43, 140)

Ownership: Municipal; supplies also about 10,000 people outside the city limits. Total population supplied, about 53,100 .

Source: Infiltration system from North Santiam River about 17 miles southeast of Salem. Auxiliary or emergency supply from wells. The wells furnished 19 percent of the total supply in 1950 .

Treatment: Chlorination and ammoniation.

Rated capacity of treatment plant: --

Raw-water storage: None.

Finished-water storage: 11,500,000 gal. A reservoir under construction will provide $100,000,000 \mathrm{gal}$ of additional storage.

The infiltration system is on Stayton Island in the North Santiam River above Stayton and below the Little North Fork. On Stayton Island 3 wells each $25 \mathrm{ft}$ deep and capable of delivering $2 \mathrm{mgd}$ are used when necessary to augment the infiltration supply. Water from the infiltration system is conducted to Salem by gravity flow through a 36 -in. pipe line.

Four wells which are in Salem and connected to the distribution system and are used during peak loads contributed about 7 percent of the supply in 1951. Contribution from all the wells probably exceeded 20 percent in 1951.

\section{ANALYSIS}

(Analysis, in parts per million, by U. S. Geological Survey)

\begin{tabular}{|c|c|c|c|}
\hline & $\begin{array}{c}\text { Finished } \\
\text { water } \\
\text { (tap sample) }\end{array}$ & & $\begin{array}{c}\text { Finished } \\
\text { water } \\
\text { (tap sample) }\end{array}$ \\
\hline $\begin{array}{l}\text { Silica }\left(\mathrm{SiO}_{2}\right) \\
\text { Iron }(\mathrm{Fe}) \\
\text { Manganese }(\mathrm{Mn})\end{array}$ & $\begin{array}{r}17.03 \\
--\end{array}$ & $\begin{array}{l}\text { Hardness as } \mathrm{CaCO}_{3}: \\
\text { Total ........................ } \\
\text { Noncarbonate ........... }\end{array}$ & $\begin{array}{c}17 \\
0\end{array}$ \\
\hline 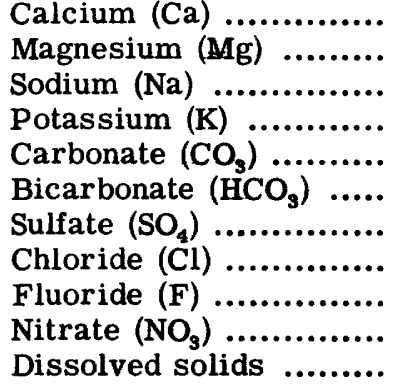 & $\begin{array}{l}4.9 \\
1.1 \\
3.2 \\
2.6 \\
0 \\
31 \\
1.7 \\
1.0 \\
.0 \\
.1\end{array}$ & 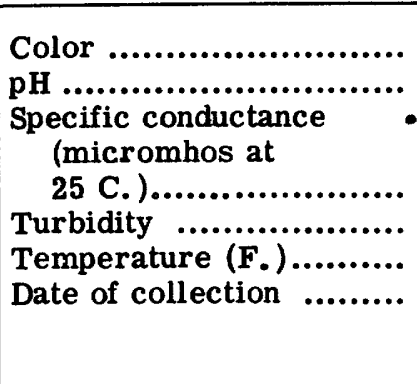 & $\begin{array}{r}54 \\
-- \\
64 \\
\text { June } 14, \\
1951\end{array}$ \\
\hline
\end{tabular}




\title{
OREGON
}

\author{
SPRINGFIELD \\ (Population, 10, 807)
}

Ownership: Mountain States Power Company.

Source: Willamette River; Auxiliary supply, 8 contiguous wells (1 to 8 ) ranging in depth from 28 to $30 \mathrm{ft}$. The yield of the wells is reported to be $1,000,400$, $1,250,1,000,150,500,900$ and $1,000 \mathrm{gpm}$ respectively. The well water is supplied directly to the transmission line which in turn feeds into the distribution system.

Treatment: Willamette River: rapid sand filtration and chlorination. During peak periods of consumption a pressure filter is operated in parallel with the 2 sand filters. The well water is chlorinated.

Rated capacity of treatment plant: 2, 500, $000 \mathrm{gpd}$.

Raw-water storage: None.

Finished-water storage: 1,550,000 gal.

The well system has been developed during 1950 and 1951. Should this source prove entirely satisfactory, it is expected to replace the Willamette River supply.

\begin{tabular}{|c|c|c|c|c|c|}
\hline & (Analy & ses, in $p$ & $\begin{array}{l}\text { LYSES } \\
\text { arts per million) }\end{array}$ & & \\
\hline & $\begin{array}{c}\text { Raw } \\
\text { water }^{a}\end{array}$ & Well $3^{b}$ & & $\begin{array}{c}\text { Raw } \\
\text { water }^{\mathrm{a}}\end{array}$ & Well $3^{b}$ \\
\hline 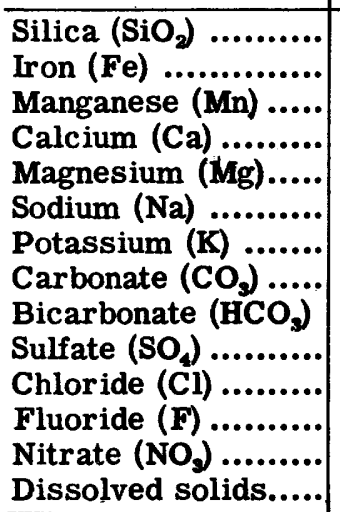 & $\begin{array}{c}17 \\
-- \\
11 \\
2.1 \\
1.4 \\
0 \\
0 \\
25 \\
15 \\
2.4 \\
-- \\
c_{61}^{--}\end{array}$ & \begin{tabular}{c|}
11 \\
1.3 \\
-- \\
11 \\
2.3 \\
3.9 \\
-- \\
-- \\
39 \\
2.5 \\
4.4 \\
-- \\
$c_{56}^{.9}$
\end{tabular} & \begin{tabular}{|c|}
$\begin{array}{c}\text { Hardness as } \mathrm{CaCO}_{3}: \\
\text { Total ................... } \\
\text { Noncarbonate...... }\end{array}$ \\
Color................... \\
pH ........................ \\
Specific conductance \\
(micromhos at \\
25 C. )................ \\
Turbidity ............. \\
Temperature (F.)... \\
Date of collection ...
\end{tabular} & $\begin{array}{r}-- \\
-- \\
-- \\
\text { Apr. } 12 \\
1951\end{array}$ & $\begin{array}{r}37 \\
5 \\
-- \\
7.1 \\
\\
-- \\
\overline{53} \\
\text { Sept. } 26, \\
1950\end{array}$ \\
\hline $\begin{array}{l}\text { Depth (feet) ...... } \\
\text { Diameter (inches } \\
\text { Date drilled ..... } \\
\text { Percent of supply }\end{array}$ & & & & & $\begin{array}{r}29 \\
12 \\
1950\end{array}$ \\
\hline
\end{tabular}

${ }^{a}$ Willamette River. Analyzed by Elgin Softener Corporation, Elgin, Illinois.

b Analyzed by Oregon State College, Corvallis, Oreg.

${ }^{c}$ Sum of determined constituents. 


\section{WASHINGTON}

\section{WASHINGTON}

\section{ABERDEEN \\ (Population, 19,653)}

Ownership: Municipal; supplies also about 1,000 people outside the city limits. Total population supplied, about 20,650 .

Source: Wishka River impounded 21. 7 miles northeast of Aberdeen. A separate industrial system, municipally owned, utilizes large quantities of raw water from the Wynooche River. Emergency supplies from Wynooche River and Lake Aberdeen.

Treatment: Chlorination and ammoniation.

Rated capacity of treatment plant: $10,000,000 \mathrm{gpd}$.

Raw-water storage: 120,000,000 gal.

Finished-water storage: 24,000,000 gal.

\section{ANALYSIS}

(Analysis, in parts per million, by U. S. Geological Survey)

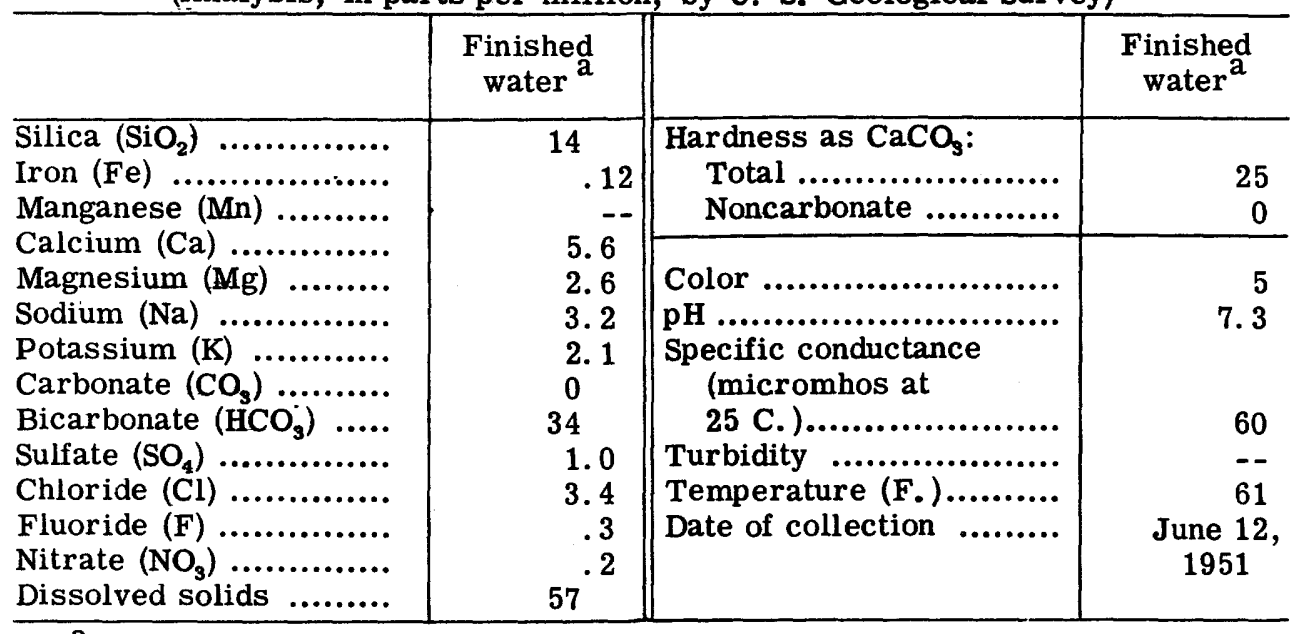

${ }^{a}$ Wishka River. 


\section{WASHINGTON}

\section{BELLINGHAM}

(Population, 34, 112)

Ownership: Municipal; supplies also about 1, 500 people outside the city limits. Total population supplied, about 35,600 .

Source: Lake Whatcom (96 percent of supply) and Lake Padden (4 percent of supply).

Treatment: Chlorination and.ammoniation. Lime added to water from Lake Whatcom for corrosion control.

Rated capacity of treatment plant: $100,000,000$ gpd.

Raw-water storage: Lake Whatcom and Lake Padden (capacities not computed).

Finished-water storage: 4 reservoirs, 2,000,000 gal; elevated tank, 75,000 gal.

\section{ANALYSIS}

(Analysis, in parts per million, by U. S. Geological Survey)

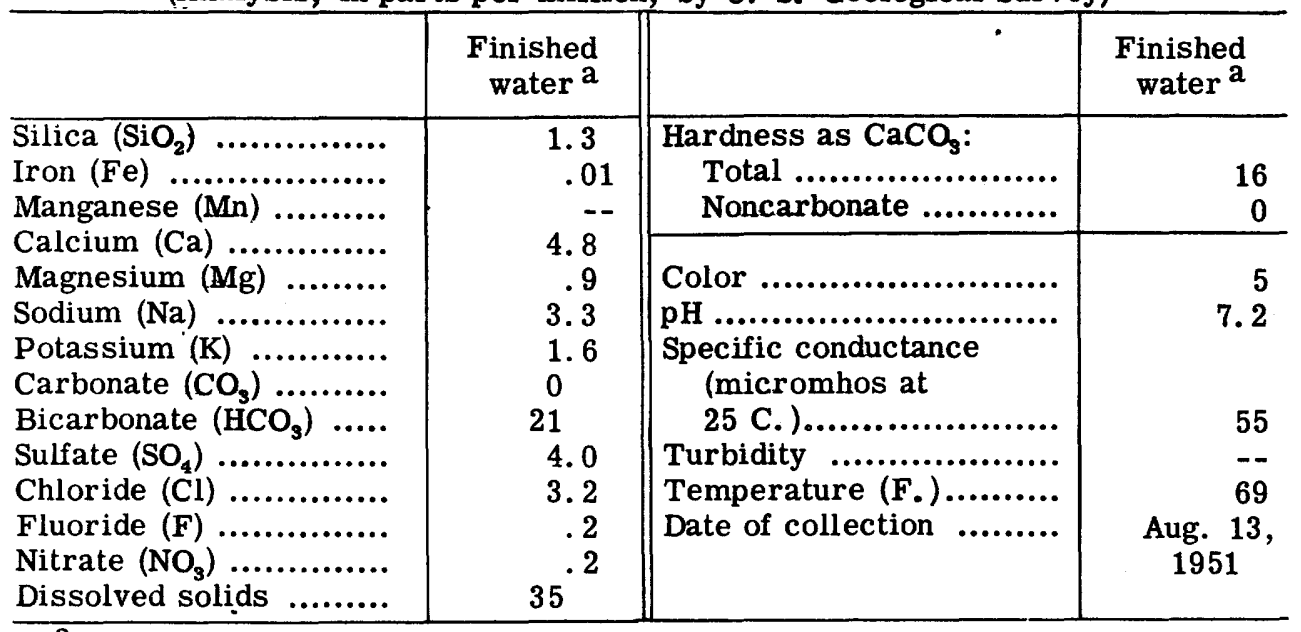

${ }^{a}$ Lake Whatcom. 


\section{WASHINGTON}

\section{BREMERTON}

(Population, 27, 678)

Ownership: Municipal; supplies also Puget Sound Naval Shipyard and military personnel, and about 20,000 people outside the city limits. Total population supplied, about 63,000 .

Source: Anderson Creek, Gorst Creek, and Union River (82 percent of supply); 7 artesian wells ( 1 to 7 ) ranging in depth from 245 to $627 \mathrm{ft}$ (18 percent of supply). The yield of the wells is reported to range from 130 to $1,500 \mathrm{gpm}$.

Treatment: Anderson and Gorst Creek stations: sedimentation, chlorination, and ammoniation; chlorination of Union River supply and at main distribution reservoir.

Rated capacity of treatment plant: 15, 000,000 gpd.

Raw-water storage: None.

Finished-water storage: Reservoirs, 22,000,000 gal.

\section{ANALYSES}

(Analyses, in parts per million, by Northwest Laboratories, Seattle, Wash.)

\begin{tabular}{|c|c|c|c|}
\hline & $\begin{array}{c}\text { Anderson } \\
\text { Creek }\end{array}$ & $\begin{array}{l}\text { Gorst } \\
\text { Creek }\end{array}$ & $\begin{array}{l}\text { Union } \\
\text { River }\end{array}$ \\
\hline 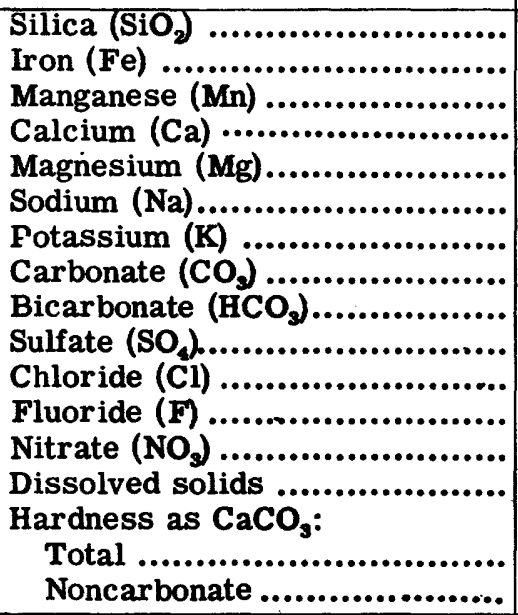 & $\begin{array}{c}39 \\
.09 \\
7.2 \\
4.2 \\
7.5 \\
-- \\
-- \\
50 \\
.8 \\
6.8 \\
.0 \\
.0 \\
90 \\
35 \\
0\end{array}$ & $\begin{array}{l}38 \\
.15 \\
-- \\
9.7 \\
4.9 \\
6.9 \\
-- \\
-- \\
58 \\
.8 \\
7.7 \\
.0 \\
.0 \\
95 \\
44 \\
0\end{array}$ & $\begin{array}{l}33 \\
.38 \\
9.0 \\
2.7 \\
12 \\
-- \\
-- \\
56 \\
2.3 \\
7.4 \\
.0 \\
.0 \\
94 \\
34 \\
0\end{array}$ \\
\hline 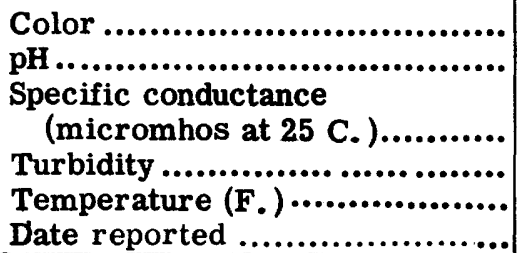 & $\begin{array}{r}2.5 \\
7.5 \\
-- \\
-- \\
\text { July } 5,1951\end{array}$ & $\begin{array}{r}0 \\
7.6 \\
-- \\
-- \\
\text { July } 5,19 \overline{51}\end{array}$ & $\begin{array}{r}6 \\
7.4 \\
-- \\
-- \\
\text { July } 5,195 \overline{1}\end{array}$ \\
\hline
\end{tabular}

${ }^{a}$ Sum of determined constituents. 
WASHENGTON

BREM ERTON--Continued

\section{ANALYSES}

(Analyses, in parts per million, by Northwest Laboratories, Seattle, Wash.)

\begin{tabular}{|c|c|c|c|}
\hline & Well 2 & Well 6 & Well 7 \\
\hline 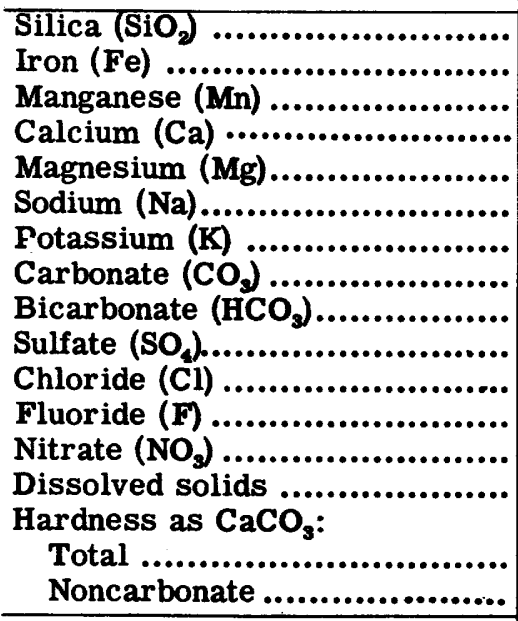 & $\begin{array}{l}31.14 \\
12 \\
5.1 \\
13 \\
79 \\
79 \\
4.4 \\
7.1 \\
.0 \\
.0 \\
106 \\
51 \\
0 \\
\end{array}$ & $\begin{array}{r}41 \\
.09 \\
15 \\
3.2 \\
9.9 \\
-- \\
76 \\
1 .- \\
6.4 \\
.0 \\
.0 \\
108 \\
52 \\
0 \\
\end{array}$ & $\begin{array}{c}30 \\
19 \\
4.05 \\
12.0 \\
12^{--} \\
17^{--} \\
114 \\
.0 \\
64 \\
0 \\
\end{array}$ \\
\hline 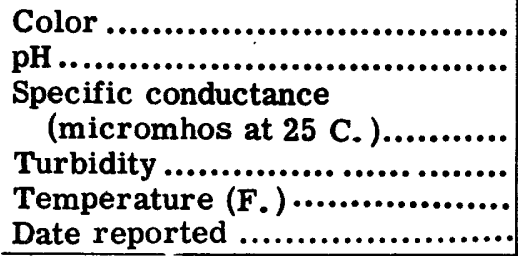 & $\begin{array}{r}0 \\
8.2 \\
-- \\
-- \\
\text { July } 5,1951\end{array}$ & $\begin{array}{r}0 \\
8.2 \\
-- \\
-- \\
-- \\
\text { July } 5,1951\end{array}$ & $\begin{array}{r}0 \\
8.1 \\
-- \\
-- \\
\text { July } 5,1951\end{array}$ \\
\hline 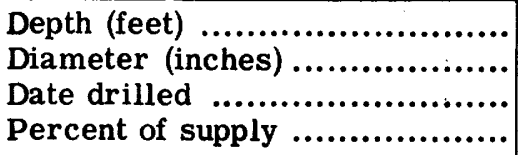 & $\begin{array}{r}245 \\
22 \\
1941 \\
--\end{array}$ & $\begin{array}{r}535 \\
16 \\
1944 \\
--\end{array}$ & $\begin{array}{r}627 \\
16 \\
1945 \\
--\end{array}$ \\
\hline
\end{tabular}




\section{WASHINGTON}

\section{EVERETT}

(Population, 33, 849)

Ownership: Municipal; supplies also about 10,000 people outside the city limits.

Total population supplied, about 43,800 .

Source: Sultan River stored in Lake Chaplain.

Treatment: Chlorination.

Rated capacity of treatment plant: $110,000,000 \mathrm{gpd}$.

Raw-water storage: Lake Chaplain, 4,500,000,000 gal.

Finished-water storage: 30,000,000 gal.

\section{ANALYSIS}

(Analysis, in parts per million, by U. S. Geological Survey)

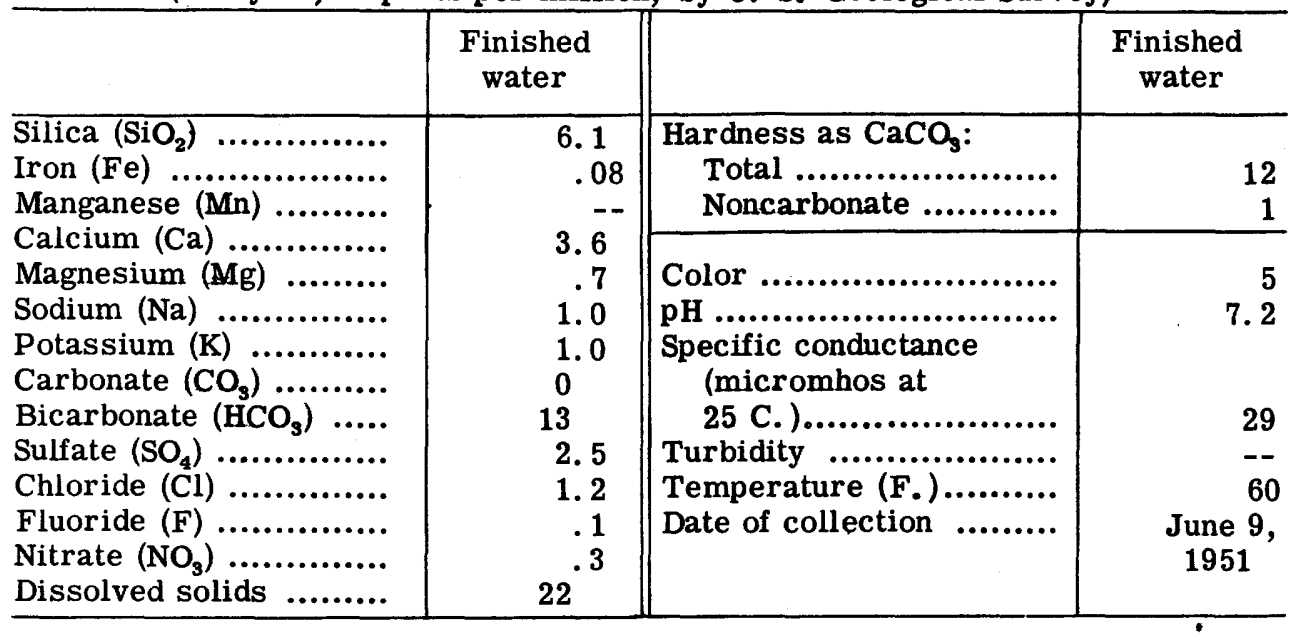




\section{WASHINGTON}

\section{LONGVIEW}

(Population, 20, 339)

Ownership: Municipal; supplies also about 4,000 people outside the city limits.

Total population supplied, about 24,300.

Source: Cowlitz River.

Treatment: Coagulation with alum and lime, sedimentation, rapid sand filtration, chlorination, and ammoniation.

Rated capacity of treatment plant: 5, 000,000 gpd.

Raw-water storage: 500,000 gal.

Finished-water storage: 7,000,000 gal.

\section{ANALYSIS}

(Analysis, in parts per million, by U. S. Geological Survey)

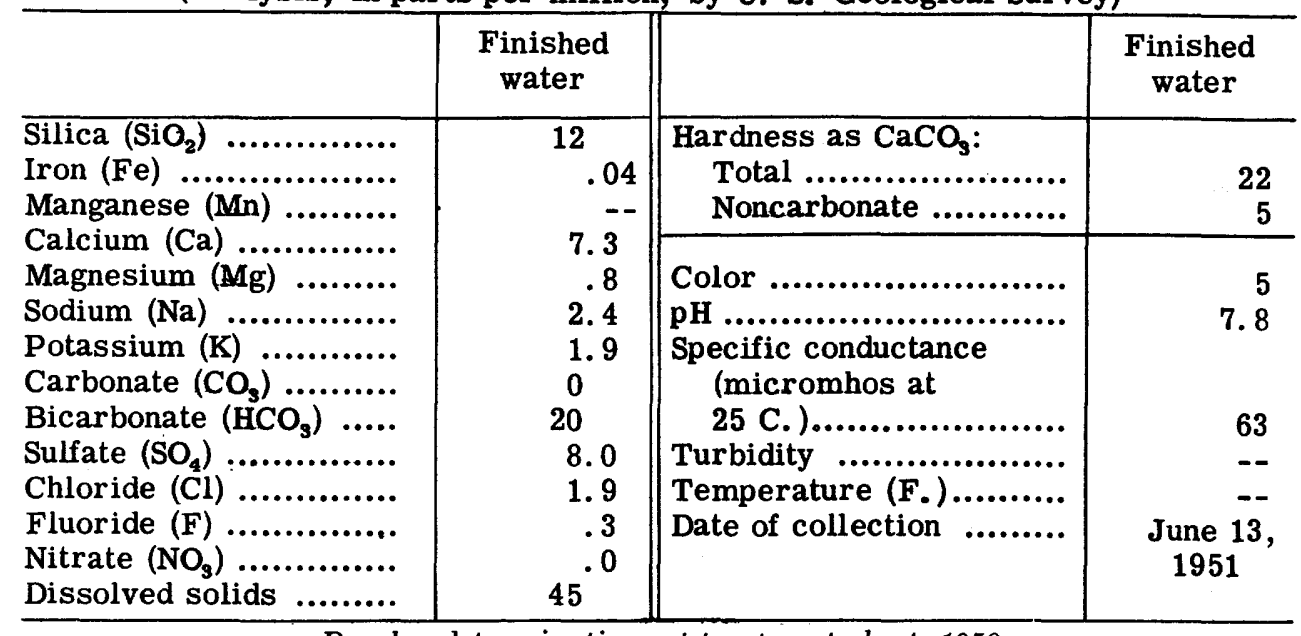

Regular determinations at treatment plant, 1950

\begin{tabular}{l|c|c|c|c|c|c|c|c|c|c|c|c}
\hline & \multicolumn{3}{|c|}{$\begin{array}{c}\text { Alkalinity } \\
\text { as CaCO } \\
\text { (ppm) }\end{array}$} & \multicolumn{3}{c|}{ pH } & \multicolumn{3}{c|}{$\begin{array}{c}\text { Hardness } \\
\text { as CaCO } \\
\text { (ppm) }\end{array}$} & \multicolumn{3}{c}{ Turbidity } \\
\cline { 2 - 13 } & Av & Max & Min & Av & Max & Min & Av & Max & Min & Av & Max & Min \\
\hline Raw water........ & -- & -- & -- & 7.0 & 7.2 & 6.8 & -- & -- & -- & 25 & 1000 & 25 \\
Finished water... & 18 & -- & -- & 7.2 & 7.4 & 7.0 & -- & -- & -- & 0 & 0 & 0 \\
\hline
\end{tabular}




\section{WASHINGTON}

\section{OLYMPIA}

(Population, 15, 819)

Ownership: Municipal; supplies also about 300 people outside the city limits.

Total population supplied, about 16,100 .

Source: McAllister Springs.

Treatment: Chlorination.

Rated capacity of treatment plant: 20, 000,000 gpd.

Raw-water storage: 2,000,000 gal.

Finished-water storage: 7, 700,000 gal.

\begin{tabular}{|c|c|c|c|}
\hline (Analysis, in pa & $s$ per millior & $\begin{array}{l}\text { YSIS } \\
\text { by U. S. Geological Surv }\end{array}$ & \\
\hline & $\begin{array}{c}\text { Finished } \\
\text { water }\end{array}$ & & $\begin{array}{c}\text { Finished } \\
\text { water }\end{array}$ \\
\hline $\begin{array}{l}\text { Silica }\left(\mathrm{SiO}_{2}\right) \ldots \ldots \ldots \ldots \ldots \\
\text { Iron }(\mathrm{Fe}) \ldots \ldots \ldots \ldots \ldots \ldots \\
\text { Manganese }(\mathrm{Mn}) \ldots \ldots \ldots \ldots\end{array}$ & $\begin{array}{r}36 \\
.03 \\
--\end{array}$ & $\begin{array}{l}\text { Hardness as } \mathrm{CaCO}_{3}: \\
\text { Total ...................... } \\
\text { Noncarbonate ........... }\end{array}$ & $\begin{array}{r}50 \\
0\end{array}$ \\
\hline 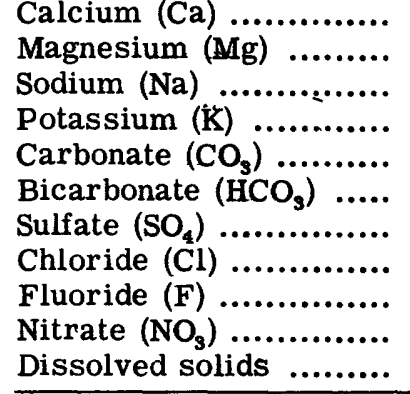 & $\begin{array}{c}10 \\
6.2 \\
6.9 \\
2.9 \\
0 \\
72 \\
3.6 \\
4.4 \\
.0 \\
.8 \\
107 \\
\end{array}$ & 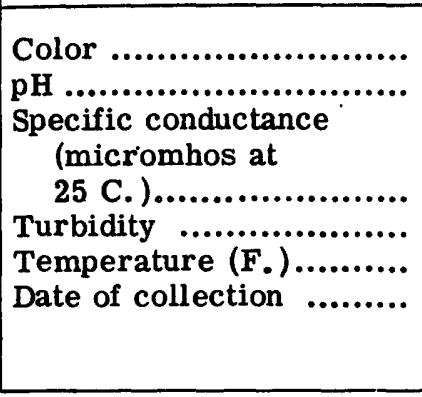 & $\begin{array}{r}133 \\
-- \\
62 \\
\text { June } 11, \\
1951\end{array}$ \\
\hline
\end{tabular}




\title{
WASHINGTON
}

\author{
RENTON \\ (Population, 16, 039)
}

Ownership: Municipal; supplies also about 1,000 people outside the city limits.

Total population supplied, about 17,000 .

Source: Springbrook Springs and 4 wells (1 and 2, and Liberty Park 1 and 2).

The yield of the wells is reported to be 105, 260, 1,000 and 1,000 gpm.

Emergency supply from City of Seattle.

Treatment: Chlorination (at source only).

Raw-water storage: $1,800,000$ gal.

Finished-water storage: 1,000,000 gal.

A private residential development of 150 homes, supplied by a private water company, has been annexed by the city. The source of the supply is springs, with a 25,000 gal storage tank. The City of Renton may purchase this water system.

\section{ANALYSES}

(Analyses, in parts per million, by U. S. Geological Survey)

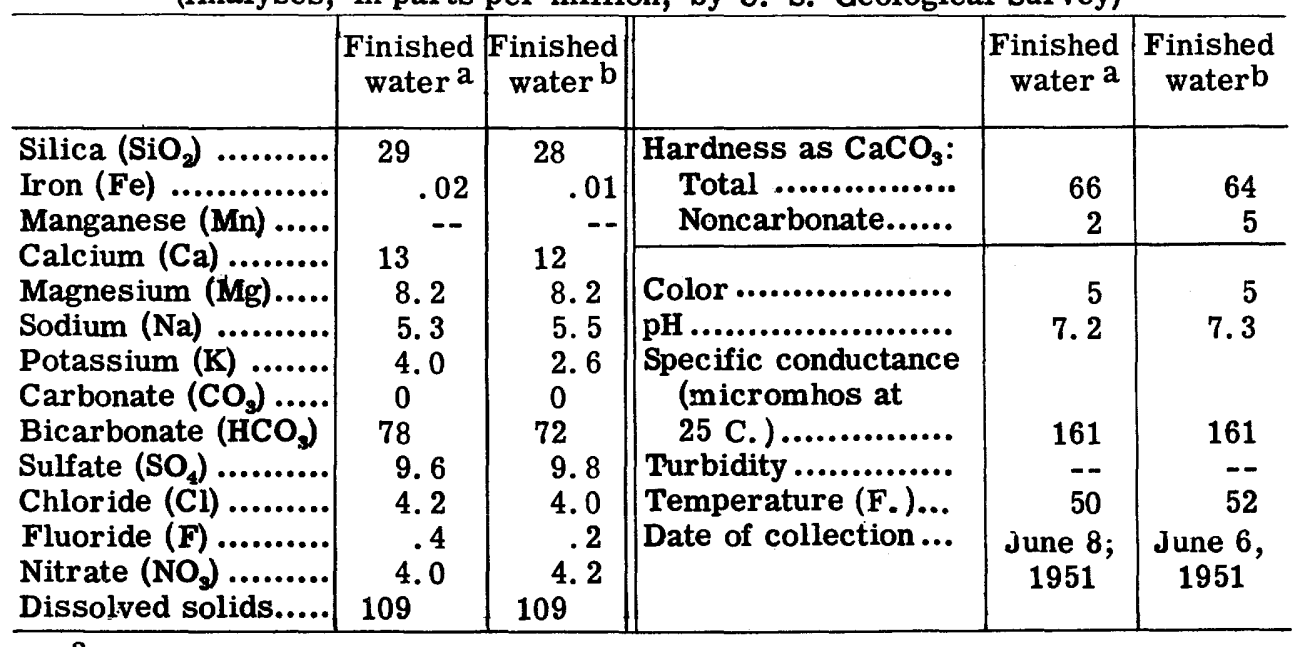

a Springbrook Springs.

${ }^{b}$ Liberty Park wells. 


\section{WASHINGTON}

\section{RICHLAND}

(Population, 21, 809)

Ownership: U. S. Government. Operated by General Electric Co. Supplies also about 6, 000 people outside Richland. Total population supplied, about 27, 800 .

Source: Domestic supply, 18 wells in four groups: Richland group of 8 wells (2, 4, $5,12,13,14,15$, and 18) ranging in depth from 70 to $140 \mathrm{ft}$, and reported to yield 6,000 gpm; North Richland group of 6 wells (A, B, C, D, E, and 5) ranging in depth from 100 to $120 \mathrm{ft}$ and reported to yield 7, $000 \mathrm{gpm}$; Columbia group of 3 wells (A, B, and C) 80 to $110 \mathrm{ft}$ deep, and reported to yield 2, 200 gpm; and 1 well (1100-8) $120 \mathrm{ft}$ deep, and reported to yield 1, $000 \mathrm{gpm}$. The Columbia group of wells is used only during the summer months.

Treatment: Chlorination of domestic supply.

Raw-water storage: --

Finished-water storage: 5, 400, 000 gal.

A separate supplementary supply for lawn sprinkling during summer months is obtained from a canal from the Yakima River through a separate distribution system. The river water is used also to flood the percolation basin in the well field area in order to maintain the ground water level during the summer months.

The water delivered to the consumers varies in chemical composition according to the wells pumped and somewhat with the level of the ground water table.

\section{ANALYSES}

(Analyses, in parts per million, by General Electric Co., Richland, Wash.)

\begin{tabular}{|c|c|c|c|}
\hline & $\begin{array}{c}8 \text { wells } \\
\text { (Richland } \\
\text { group) }\end{array}$ & $\begin{array}{l}6 \text { wells } \\
\text { (North Rich- } \\
\text { land group) }\end{array}$ & $\begin{array}{c}\text { Well } \\
1100-8\end{array}$ \\
\hline Silica $\left(\mathrm{SiO}_{2}\right) \ldots \ldots \ldots \ldots \ldots \ldots \ldots \ldots$ & & 38 & 52 \\
\hline Iron $(\mathrm{Fe})$ & .01 & .02 & .00 \\
\hline Manganese (Mn). & .00 & .00 & $<.01$ \\
\hline 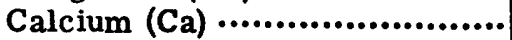 & & 27 & 34 \\
\hline Magnesium (Mg).................... & 16 & 5.8 & 7.5 \\
\hline Sodium (Na)........................... & 21 & 8.5 & 16 \\
\hline Potassium (K) ............ & 4 & 1.3 & 2.7 \\
\hline Carbonate $\left(\mathrm{CO}_{2}\right)$......................... & & -- & \\
\hline Bicarbonate $\left(\mathrm{HCO}_{3}\right) \ldots \ldots \ldots \ldots \ldots$ & 206 & 100 & 139 \\
\hline Sulfate $\left(\mathrm{SO}_{4}\right) . . . \ldots \ldots \ldots \ldots \ldots \ldots \ldots$ & 44 & 12 & 18 \\
\hline Chloride (Cl) $\ldots \ldots \ldots \ldots \ldots \ldots \ldots \ldots$ & 8.5 & 4.0 & 5.5 \\
\hline Fluoride $(F) \ldots \ldots \ldots \ldots \ldots \ldots \ldots$ & .2 & .2 & .2 \\
\hline Nitrate $\left(\mathrm{NO}_{3}\right) \ldots \ldots \ldots \ldots \ldots \ldots \ldots$ & 9 & 8 & 3 \\
\hline Dissolved solids ......... & 307 & 148 & 186 \\
\hline Hardness as $\mathrm{CaCO}_{3}$ : & & & \\
\hline 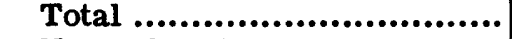 & 191 & 91 & 116 \\
\hline Noncarbonate ....................... & 22 & 9 & 2 \\
\hline Color ............... & 5 & 0 & \\
\hline pH $\ldots \ldots \ldots \ldots \ldots \ldots \ldots \ldots . . . . . . . . . .$. & 7.7 & 7.8 & 7.8 \\
\hline $\begin{array}{l}\text { Specific conductance } \\
\text { (micromhos at } 25 \text { C. ).... }\end{array}$ & 424 & 202 & 283 \\
\hline Turbidity ............................. & 2 & $<1$ & $<.1$ \\
\hline Temperature (F.) ........... & & $010=1$ & $10-$ \\
\hline Date of collection ....... & Apr. 24, 1951 & Apr. 24, 1951 & Apr. 24, 1951 \\
\hline
\end{tabular}


WASHINGTON

RICHLAND--Continued

\begin{tabular}{|c|c|c|c|}
\hline & $\begin{array}{c}8 \text { wells } \\
\text { (Richland } \\
\text { group) }\end{array}$ & $\begin{array}{l}6 \text { wells } \\
\text { (North Rich- } \\
\text { land group) }\end{array}$ & $\begin{array}{c}\text { Well } \\
1100-8\end{array}$ \\
\hline 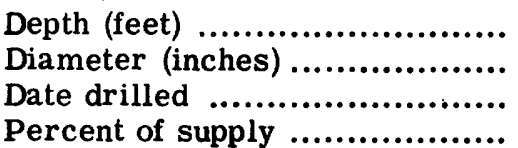 & $\begin{array}{r}70 \text { to } 140 \\
16 \text { to } 30 \\
1943-44\end{array}$ & $\begin{array}{r}100 \text { to } 120 \\
20 \text { to } 30 \\
1948 \\
--\end{array}$ & $\begin{array}{r}120 \\
10 \\
1948 \\
--\end{array}$ \\
\hline
\end{tabular}

Regular determinations at treatment plant, 1950

\begin{tabular}{|c|c|c|c|c|c|c|c|c|c|c|c|c|}
\hline & \multicolumn{3}{|c|}{$\begin{array}{c}\text { Alkalinity } \\
\text { as CaCO } \\
\text { (ppm) }\end{array}$} & \multicolumn{3}{|c|}{ pH } & \multicolumn{3}{|c|}{$\begin{array}{c}\text { Hardness } \\
\text { as } \mathrm{CaCO}_{3} \\
\text { (ppm) }\end{array}$} & \multicolumn{3}{|c|}{ Turbidity } \\
\hline & Av & Max & Min & Av & Max & Min & Av & Max & Min & Av & Max & Min \\
\hline $\begin{array}{l}\text { Raw water......... } \\
\text { Finished water... }\end{array}$ & $\begin{array}{r}-- \\
120\end{array}$ & $\overrightarrow{232}$ & $\overline{80}$ & $7 . \overline{2}$ & $7 \overline{-}$ & 7.0 & $\overline{140}$ & 202 & $\overline{90}$ & $2 . \overline{0}$ & $2 .--$ & $2 . \overline{0}$ \\
\hline
\end{tabular}




\title{
WASHINGTON
}

\author{
SEATTLE \\ (Population, 467, 591)
}

Ownership: Municipal; supplies also about 110,000 people outside the city limits.

Total population supplied, about 580,000 .

Source: Cedar River impounded in Lake Youngs.

Treatment: Chlorination and ammoniation.

Rated capacity of treatment plant: Approximately 300, 000, 000 gpd.

Raw-water storage: Lake Youngs, 3,660,000,000 gal.

Finished-water storage: $365,000,000 \mathrm{gal}$.

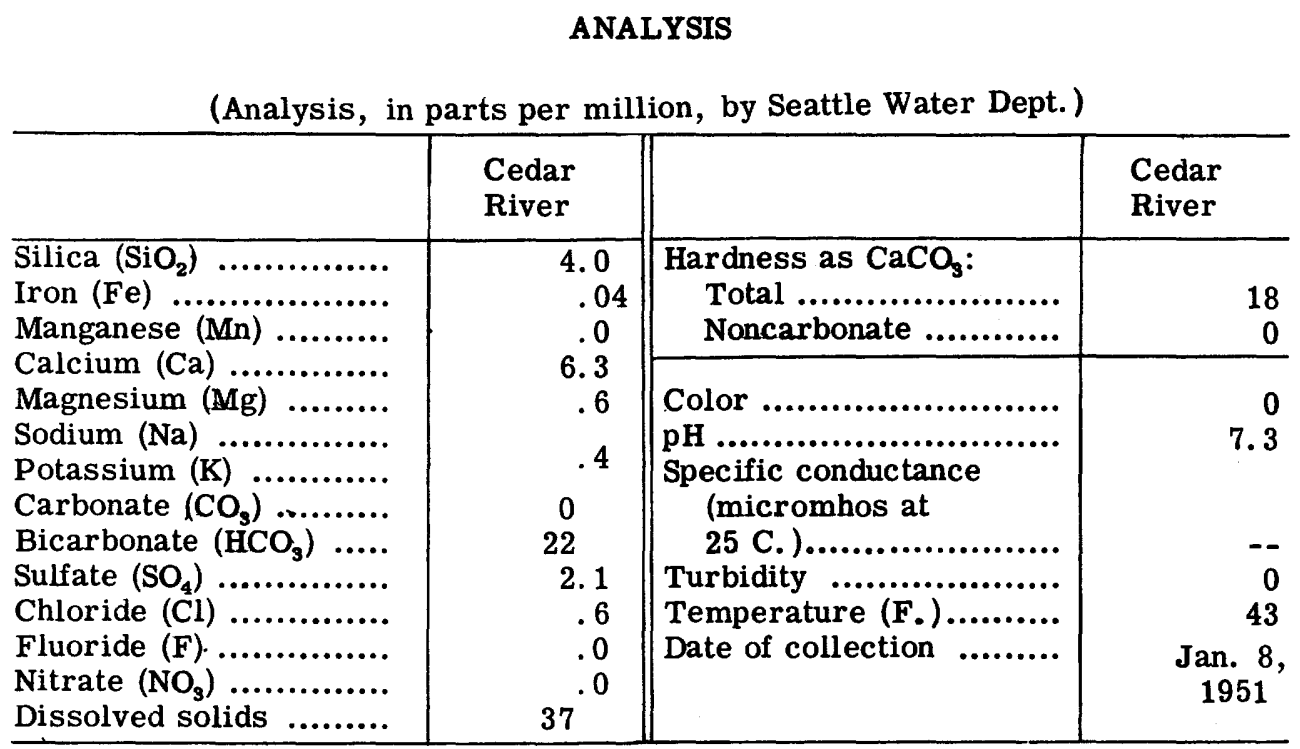




\title{
WASHINGTON
}

\author{
SPOKANE \\ (Population, 161, 721)
}

Ownership: Municipal; supplies also about 400 people outside the city limits.

Total population supplied, about 162,100 .

Source: 13 wells in two groups (87 percent of supply) with two pumping stations:

Well Pumping Station (5 wells of $45 \mathrm{ft}$ mean depth) and Parkwater Pumping

Station (8 wells of $140 \mathrm{ft}$ mean depth). Seven auxiliary wells (13 percent of supply) with four pumping stations: Ray Street (2 wells of $76 \mathrm{ft}$ mean depth);

Grace Avenue (1 well); Hoffman Avenue (2 wells of $225 \mathrm{ft}$ mean depth); and

Baxter (2 wells of $130 \mathrm{ft}$ mean depth). The auxiliary wells are used during the summer months.

Treatment: Chlorination.

Rated capacity of treatment plant: --

Raw-water storage: --

Finished-water storage: 70,100,000 gal.

\section{ANALYSES}

(Analyses, in parts per million, by City of Spokane Health Dept.)

\begin{tabular}{|c|c|c|c|c|c|}
\hline & $\begin{array}{c}\text { Well } \\
\text { Station a }\end{array}$ & \begin{tabular}{|c|} 
Park- \\
water \\
Station $^{b}$
\end{tabular} & & $\begin{array}{c}\text { Well } \\
\text { Station a }\end{array}$ & $\begin{array}{c}\text { Park- } \\
\text { water } \\
\text { Station }\end{array}$ \\
\hline $\begin{array}{l}\text { Silica }\left(\mathrm{SiO}_{2}\right) \\
\text { Iron }(\mathrm{Fe}) \ldots \ldots \ldots \ldots \\
\text { Manganese }(\mathrm{Mn}) \ldots \ldots\end{array}$ & $\begin{array}{r}20 \\
.0 \\
.0\end{array}$ & $\begin{array}{r}11 \\
.0 \\
.0\end{array}$ & $\begin{array}{c}\text { Hardness as } \mathrm{CaCO}_{3} \text { : } \\
\text { Total ................... } \\
\text { Noncarbonate..... }\end{array}$ & $\begin{array}{r}153 \\
10\end{array}$ & $\begin{array}{r}161 \\
17\end{array}$ \\
\hline $\begin{array}{l}\text { Calcium }(\mathrm{Ca}) . . . . . . . \\
\text { Magnesium }(\mathrm{Mg}) . . .\end{array}$ & $\begin{array}{l}35 \\
16\end{array}$ & $\begin{array}{l}38 \\
16\end{array}$ & Color ....................... & -- & -- \\
\hline Sodium (Na) ........... & 4.0 & 4.9 & pH $\ldots . . . \ldots \ldots \ldots . . . . . . . . .$. & 7.9 & 7.9 \\
\hline $\begin{array}{l}\text { Potassium }(\mathrm{K}) \\
\text { Carbonate }(\mathrm{CO}, \ldots \ldots\end{array}$ & $0^{--}$ & $0^{--}$ & $\begin{array}{l}\text { Specific conductance } \\
\text { (micromhos at }\end{array}$ & & \\
\hline Bicarbonate $\left(\mathrm{HCO}_{3}\right)$ & 175 & 175 & 25 C. $) \ldots \ldots \ldots \ldots \ldots$ & -- & - \\
\hline Sulfate $\left(\mathrm{SO}_{4}\right) \ldots . . . . .$. & 13 & 13 & Turbidity ............... & -- & - \\
\hline Chloride (Cl). & 2.0 & 1.9 & Temperatu & -- & -- \\
\hline Fluo & 0 & .0 & Date of collection... & June & June \\
\hline $\begin{array}{l}\text { Nitrate }\left(\mathrm{NO}_{3}\right) \ldots . . . . . . \\
\text { Dissolved solids..... }\end{array}$ & $184^{2.2}$ & $\begin{array}{r}2.2 \\
186\end{array}$ & & 1951 & 1951 \\
\hline \multirow{4}{*}{\multicolumn{4}{|c|}{ 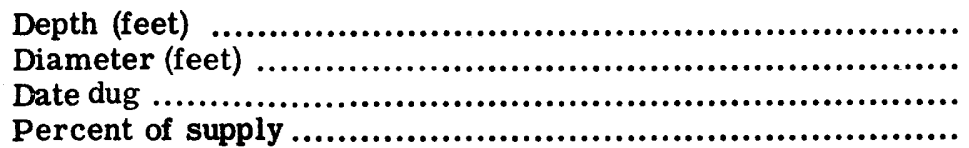 }} & & 140 \\
\hline & & & & 28 to 50 & \\
\hline & & & & $1907-25$ & 1948 \\
\hline & & & & & \\
\hline
\end{tabular}

a Five wells.
b Eight wells. 


\section{WASHINGTON}

\section{SPOKANE--Continued}

\section{ANALYSES}

(Analyses, in parts per million, by City of Spokane Health Dept.)

\begin{tabular}{|c|c|c|c|c|}
\hline & $\begin{array}{l}\text { Ray Street } \\
\text { Station } \\
\text { (2 wells) } \\
\end{array}$ & \begin{tabular}{|c|} 
Hoffman \\
Avenue Sta- \\
tion (2 wells) \\
\end{tabular} & $\begin{array}{l}\text { Baxter } \\
\text { Station } \\
\text { (2 wells) } \\
\end{array}$ & $\begin{array}{c}\text { Grace } \\
\text { Avenue } \\
\text { Well } \\
\end{array}$ \\
\hline Silica $\left(\mathrm{SiO}_{2}\right) \ldots$ & 7.2 & 7.2 & 6.4 & 15 \\
\hline Iron $(\mathrm{Fe}) \ldots \ldots \ldots \ldots \ldots \ldots \ldots \ldots$ & 0 & .0 & .0 & -- \\
\hline Manganese (Mn) .................. & -- & -- & - $\quad--$ & .0 \\
\hline 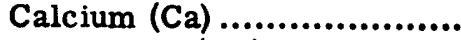 & 39 & 32 & 37 & 29 \\
\hline Magnesium $(\mathrm{Mg})$................ & 12 & 15 & 13 & 11 \\
\hline Sodium (Na)............ & 14 & 1.0 & 4.1 & 3.0 \\
\hline Potassium (K) .. & -- & -- & 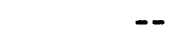 & -- \\
\hline Carbonate $\left(\mathrm{CO}_{3}\right) \ldots \ldots \ldots \ldots . . . . .$. & $\mathbf{0}$ & 0 & 0 & 0 \\
\hline Bicarbonate $\left(\mathrm{HCO}_{3}\right) . . . . . . . . . . .$. & 176 & 146 & 145 & 126 \\
\hline Sulfate $\left(\mathrm{SO}_{4}\right) \ldots \ldots \ldots \ldots \ldots \ldots$ & 22 & 16 & 27 & 12 \\
\hline 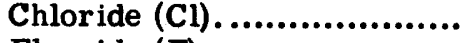 & 7.4 & 2.5 & 5.7 & 3.6 \\
\hline Fluoride (F) & -- & -- & -- & .0 \\
\hline Nitrate $\left(\mathrm{NO}_{3}\right) \ldots \ldots \ldots \ldots \ldots \ldots \ldots$ & 8.9 & .9 & & 4.0 \\
\hline Dissolved solids ................ & 191 & 144 & 195 & 132 \\
\hline Hardness as $\mathrm{CaCO}_{3}$ : & & & & \\
\hline 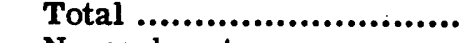 & 147 & 142 & 146 & 118 \\
\hline Noncarbonate ... & 2 & 22 & 27 & 14 \\
\hline Color................. & -- & -- & -- & -- \\
\hline pH... & 7.6 & 8.1 & 8.2 & 7.9 \\
\hline Specific conductan & & & & \\
\hline (micromhos at $25 \mathrm{C}$. ) $\ldots . .$. & -- & -- & -- & -- \\
\hline Turbidity ............. & -- & -- & -- & - \\
\hline Temperature (F.) & $0-\overline{1}$ & -- & -- & 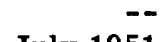 \\
\hline Date of collection ................ & Oct. 1945 & Sept. 1944 & Dec. 1944 & July 1951 \\
\hline Deptl & 76 & 225 & 130 & -- \\
\hline er (feet) & 20 & 5 & & -- \\
\hline ug .......... & 1937 & 1923 & 1943 & -- \\
\hline Percent of supply...$\ldots \ldots \ldots \ldots \ldots$ & 6 & 3 & .3 & 4 \\
\hline
\end{tabular}




\section{WASHINGTON}

\section{TACOMA}

(Population, 143, 673)

Ownership: Municipal; supplies also about 12,000 people outside the city limits. Total population supplied, about 155,700 .

Source: Green River (94 percent of supply); auxiliary supply from 13 wells ranging in depth from 74 to $788 \mathrm{ft}$, and an average yield (reported) of 3,490 gpm.

The well supply is used when the Green River is turbid, during peak demand for sprinkling, and for emergencies.

Treatment: Chlorination and ammoniation.

Rated capacity of treatment plant: $72 ; 000,000 \mathrm{gpd}$.

Finished-water storage: Reservoirs, 160,000,000 gal; standpipes, 2, 574,000 gal.

The intake on Green River is near Palmer, about 35 miles east of Tacoma. The water is transmitted by pipe line to McMillin Reservoir, located 8 miles southeast of Tacoma. A second pipe line connects McMillin Reservoir and the distribution system in Tacoma.

\section{ANALYSES}

(Analyses, in parts per million, by Northwest Laboratories, Seattle, Wash.)

\begin{tabular}{|c|c|c|c|}
\hline & $\begin{array}{c}\text { Green River } \\
\text { (raw water) }\end{array}$ & $\begin{array}{c}\text { Finished } \\
\text { water }\end{array}$ & Well 5-A \\
\hline 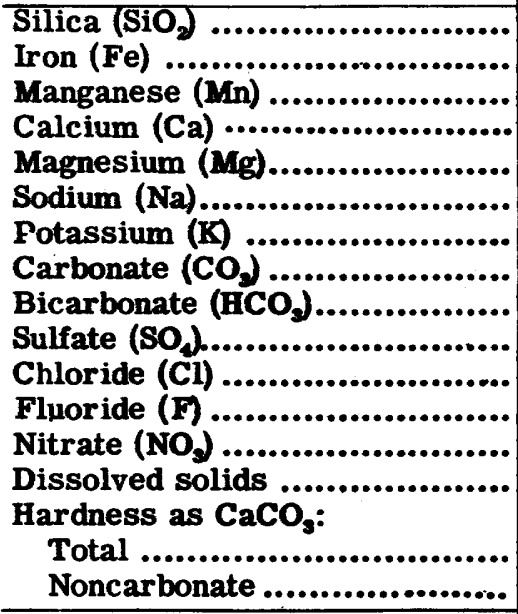 & $\begin{array}{c}23 \\
0 \\
0 \\
5.2 \\
1.2 \\
6.9 \\
-- \\
29 \\
2 .- \\
2.0 \\
6.3 \\
0 \\
0 \\
29 \\
18 \\
0 \\
\end{array}$ & $\begin{array}{c}26 \\
0 \\
0 \\
6.4 \\
1.3 \\
6.7 \\
-- \\
-- \\
30 \\
2.1 \\
6.3 \\
0 \\
0 \\
a_{64} \\
21 \\
0 \\
\end{array}$ & $\begin{array}{r}27 \\
0 \\
0 \\
12 \\
7.7 \\
9.4 \\
-- \\
-- \\
56 \\
8.9 \\
9.5 \\
0 \\
17 \\
115 \\
61 \\
16 \\
\end{array}$ \\
\hline 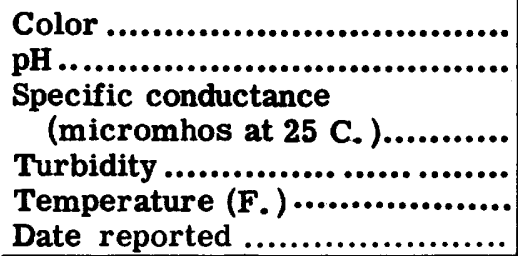 & $\begin{array}{r}0 \\
7.4 \\
-- \\
-- \\
\text { Oct. } 4,1948\end{array}$ & $\begin{array}{r}0 \\
7.8 \\
-- \\
-- \\
\text { Oct. } 4,1948\end{array}$ & $\begin{array}{r}0 \\
7.1 \\
-- \\
-- \\
\text { Oct. } 4,1948\end{array}$ \\
\hline $\begin{array}{l}\text { Depth (feet) ............ } \\
\text { Diameter (inches) .... } \\
\text { Date drilled ............ } \\
\text { Percent of supply .... }\end{array}$ & & & $\begin{array}{r}378 \\
26 \\
1930\end{array}$ \\
\hline
\end{tabular}

${ }^{\mathrm{a}}$ Sum of determined constituents. 


\section{WASHINGTON}

TACOMA--Continued

\begin{tabular}{|c|c|c|c|c|c|c|c|c|c|c|c|c|}
\hline \multicolumn{13}{|c|}{ Regular determinations at treatment plant } \\
\hline & \multicolumn{3}{|c|}{$\begin{array}{l}\text { Alkalinity } \\
\text { as } \mathrm{CaCO}_{3} \\
\text { (ppm) }\end{array}$} & \multicolumn{3}{|c|}{ pH } & \multicolumn{3}{|c|}{$\begin{array}{c}\text { Hardness } \\
\text { as CaCO } \\
\text { (ppm) }\end{array}$} & \multicolumn{3}{|c|}{ Turbidity } \\
\hline & Av & $\operatorname{Max}$ & Min & $\mathbf{A v}$ & $\operatorname{Max}$ & Min & Av & $\operatorname{Max}$ & Min & $\mathbf{A v}$ & $\operatorname{Max}$ & Min \\
\hline Raw water. & - & - & -- & 7.8 & 7.8 & 7.1 & 21 & 61 & 18 & 0 & 150 & 0 \\
\hline Finished water. & -- & -- & -- & $\ldots$ & -- & -- & -- & -- & -- & -- & -- & -- \\
\hline
\end{tabular}

VANCOUVER

(Population, 41, 664)

Ownership: Municipal; supplies also about 6,800 people outside the city limits.

Total population supplied, about 48,500 .

Source: 14 wells (72 percent of supply) and springs (28 percent of supply). The range in depth and reported yield of 5 wells $(1,3,4,5$, and 6$)$ represent about average conditions for the 14 wells. The depth and the yield of the 5 wells are as follows: well $1,132 \mathrm{ft}$ and $1,000 \mathrm{gpm}$; well $3,128 \mathrm{ft}$ and 1,000 gpm; well 4,243 ft and 2,000 gpm; well 5, $240 \mathrm{ft}$ and 1,200 gpm; well 6, $278 \mathrm{ft}$ and 2,000 gpm.

Treatment: Chlorination.

Raw-water storage: - -

Finished-water storage: $5,728,000$ gal.

The analysis given is representative of the water furnished to the consumers.

\section{ANALYSIS}

(Analysis, in parts per million, by Charlton Laboratories, Portland, Oreg.)

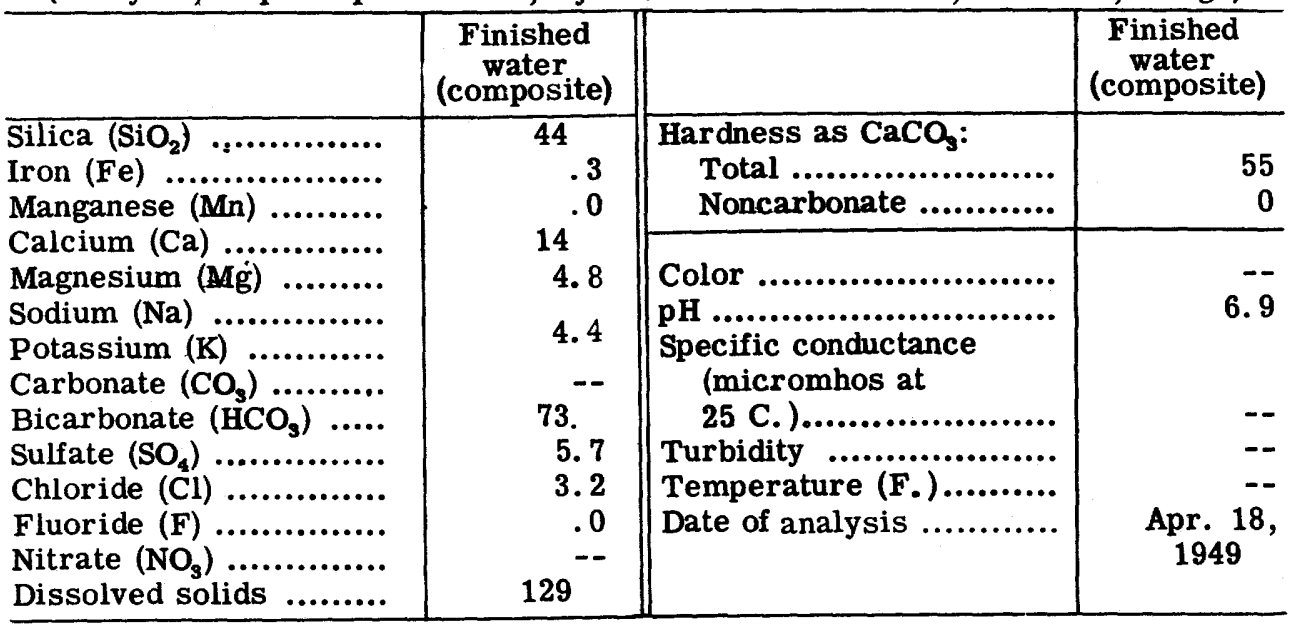




\section{WASHINGTON}

WALLA WALLA

(Population, 24, 102)

Ownership: Municipal; supplies also about 1,500 people outside the city limits. Total population supplied, about 25,600 .

Source: Mill Creek (84 percent of supply); auxiliary supply from 3 wells ( 1 to 3 ), 810,808 , and $1,169 \mathrm{ft}$ deep. The yield of the wells is reported to be 1,500 , 1,200 , and 2,000 gpm. The wells are pumped only during the summer months and furnish 16 percent of the annual supply.

Treatment: Chlorination of water from Mill Creek; well supply not treated. Raw-water storage: 15, 000, 000 gal.

Finished-water storage:

\section{ANALYSIS}

(Analysis, in parts per million, by U. S. Geological Survey)

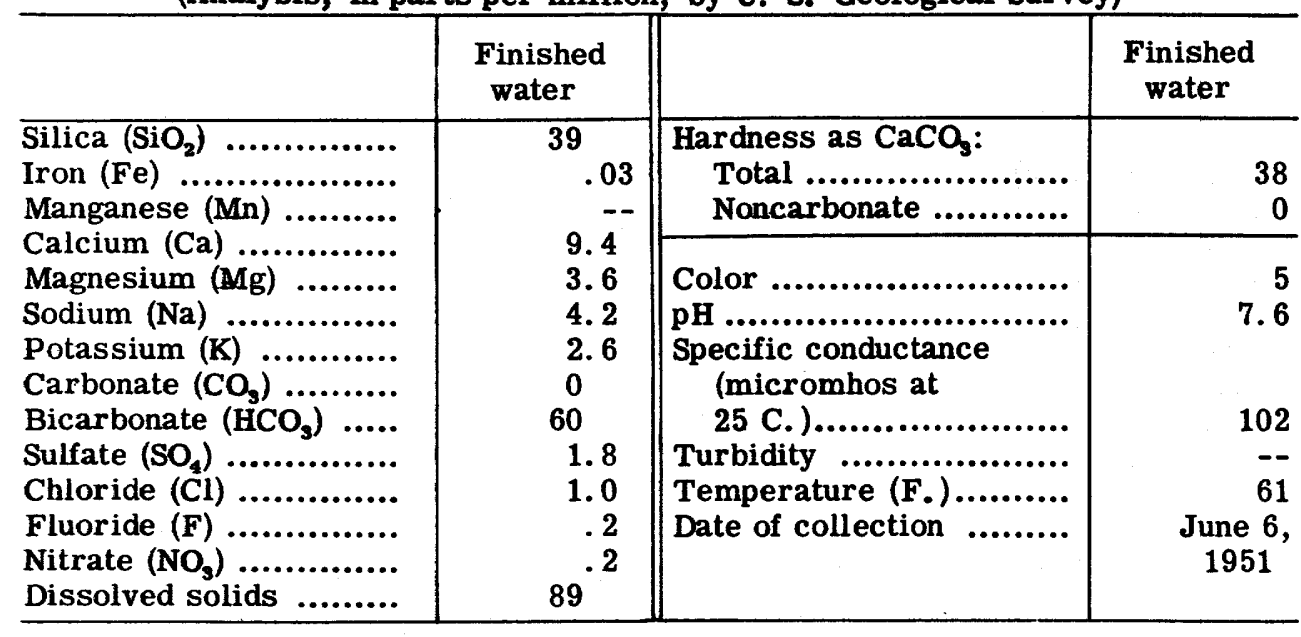




\section{WASHINGTON}

WENATCHEE

(Population, 13, 072)

Ownership: Municipal; supplies also about 2,000 people outside the city limits. Total population supplied, about 15,100 .

Source: Columbia River.

Treatment: Sedimentation, coagulation with alum, rapid sand filtration, and chlorination.

Rated capacity of treatment plant: 14,000,000 gpd.

Raw-water storage: None.

Finished-water storage: 4,000,000 gal.

\section{ANALYSIS}

(Analysis, in parts per million, by U. S. Geological Survey)

\begin{tabular}{|c|c|c|c|c|c|c|c|c|c|c|c|c|}
\hline & \multicolumn{2}{|c|}{$\begin{array}{c}\text { Columbia } \\
\text { River } \\
\text { (raw water) }\end{array}$} & & \multicolumn{2}{|c|}{$\begin{array}{c}\text { Columbia } \\
\text { River } \\
\text { (raw water) }\end{array}$} \\
\hline \multirow{8}{*}{\multicolumn{3}{|c|}{ 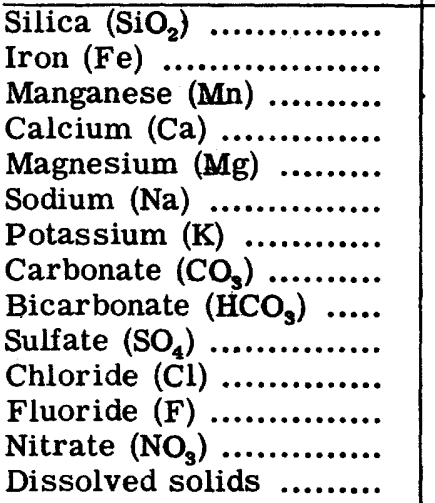 }} & \multirow{3}{*}{\multicolumn{2}{|c|}{$\begin{array}{c}9.6 \\
-- \\
20 \\
3.6 \\
8.6\end{array}$}} & \multicolumn{6}{|c|}{ 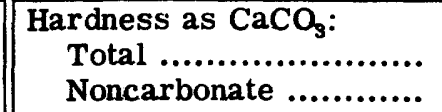 } & \multicolumn{2}{|r|}{$\begin{array}{r}65 \\
65\end{array}$} \\
\hline & & & & & \multirow{7}{*}{\multicolumn{6}{|c|}{ 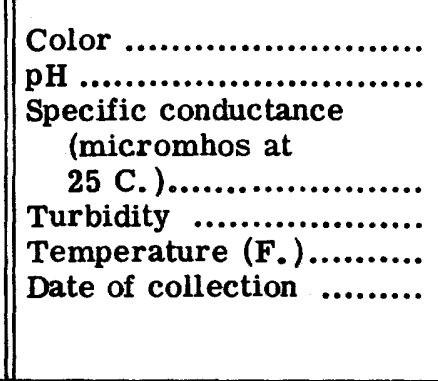 }} & & \\
\hline & & & & & & & & & & & & \\
\hline & & & \multirow{5}{*}{\multicolumn{2}{|c|}{$\begin{array}{r}0 \\
72 \\
21 \\
1 \\
.2 \\
\mathrm{a}_{100}^{.6}\end{array}$}} & & & & & & & & \\
\hline & & & & & & & & & & & & \\
\hline & & & & & & & & & & & & \\
\hline & & & & & & & & & & & & \\
\hline \multirow{2}{*}{\multicolumn{13}{|c|}{ Regular determinations at treatment plant, 195}} \\
\hline & & & & & & & & & & & & \\
\hline & \multicolumn{3}{|c|}{$\begin{array}{l}\text { Alkalinity } \\
\text { as CaCO } \\
\text { (ppm) }\end{array}$} & \multicolumn{3}{|c|}{$\mathrm{pH}$} & \multicolumn{3}{|c|}{$\begin{array}{l}\text { Hardness } \\
\text { as } \mathrm{CaCO}_{3} \\
(\mathrm{ppm})\end{array}$} & \multicolumn{3}{|c|}{ Turbidity } \\
\hline & Av & $\operatorname{Max}$ & $\mathrm{Min}$ & Av & $\operatorname{Max}$ & $\mathrm{Min}$ & Av & Max & Min & Av & $\operatorname{Max}$ & Min \\
\hline Raw water..... & 45 & 51 & 39 & 7.7 & 8.1 & 7.3 & 45 & 60 & 30 & 2.3 & 3.5 & 1.0 \\
\hline & 45 & 50 & 41 & 7.5 & 7.8 & 7.3 & 46 & 60 & 32 & .2 & .5 & 0 \\
\hline
\end{tabular}

${ }^{\mathrm{a}}$ Sum of determined constituents. 


\section{WASHINGTON}

YAKDMA

(Population, 38, 486)

Ownership: Municipal; supplies also about 3,800 people outside the city limits. Total population supplied, about 42,300 .

Source: Naches River; emergency supply from 2 wells (1 and 2) 250 and $65 \mathrm{ft}$ deep. (No well water was used during 1950.)

Treatment: Plain sedimentation, filtration through natural sand and gravel beds, and chlorination.

Rated capacity of treatment plant: --

Raw-water storage: --

Finished-water storage: 24,000,000 gal.

\section{ANALYSIS}

(Analysis, in parts per million, by U. S. Geological Survey)

\begin{tabular}{|c|c|c|c|}
\hline & $\begin{array}{c}\text { Finished } \\
\text { water }^{2}\end{array}$ & & $\begin{array}{r}\text { Finished } \\
\text { water }^{2}\end{array}$ \\
\hline $\begin{array}{l}\text { Silica }\left(\mathrm{SiO}_{2}\right) \\
\text { Iron }(\mathrm{Fe}) \\
\text { Manganese }(\mathrm{Mn}) \ldots \ldots \ldots \ldots \ldots \ldots\end{array}$ & $\begin{array}{l}17.11 \\
.-\end{array}$ & $\begin{array}{r}\text { Hardness as } \mathrm{CaCO}_{3}: \\
\text { Total ......................... } \\
\text { Noncarbonate ............. }\end{array}$ & $\begin{array}{r}21 \\
0\end{array}$ \\
\hline $\begin{array}{l}\text { Calcium }(\mathrm{Ca}) \\
\text { Magnesium }(\mathrm{Mg}) \\
\text { Sodium }(\mathrm{Na}) \\
\text { Potassium }(\mathrm{K})\end{array}$ & $\begin{array}{c}6.1 \\
1.4 \\
3.0 \\
1.0 \\
0 \\
30 \\
2.5 \\
1.7 \\
.1 \\
.1 \\
50\end{array}$ & 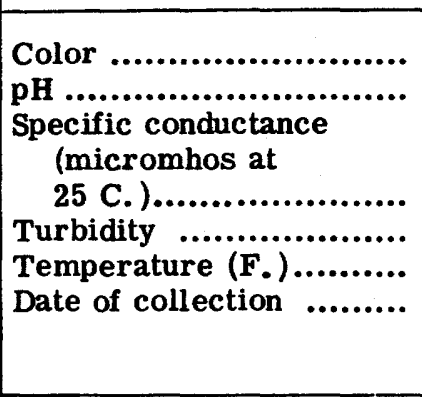 & $\begin{array}{r}57 \\
-- \\
64 \\
\text { June } 7, \\
1951\end{array}$ \\
\hline
\end{tabular}

${ }^{\text {a }}$ Naches River. 
